\title{
Adaptive-network models of collective dynamics
}

\author{
DISSERTATION \\ zur Erlangung des akademischen Grades \\ Doctor rerum naturalium \\ (Dr. rer. nat.) \\ vorgelegt \\ der Fakultät Mathematik und Naturwissenschaften \\ der Technischen Universität Dresden \\ von \\ Gerd Zschaler \\ geb. am 22.02.1982 in Dresden
}


1. Gutachter: Prof. Dr. Frank Jülicher

2. Gutachter: Prof. Kevin E. Bassler

Tag der Disputation: 15.05.2012 


\section{Abstract}

Complex systems can often be modelled as networks, in which their basic units are represented by abstract nodes and the interactions among them by abstract links. This network of interactions is the key to understanding emergent collective phenomena in such systems. In most cases, it is an adaptive network, which is defined by a feedback loop between the local dynamics of the individual units and the dynamical changes of the network structure itself. This feedback loop gives rise to many novel phenomena. Adaptive networks are a promising concept for the investigation of collective phenomena in different systems. However, they also present a challenge to existing modelling approaches and analytical descriptions due to the tight coupling between local and topological degrees of freedom.

In this thesis, I present a simple rule-based framework for the investigation of adaptive networks, using which a wide range of collective phenomena can be modelled and analysed from a common perspective. In this framework, a microscopic model is defined by the local interaction rules of small network motifs, which can be implemented in stochastic simulations straightforwardly. Moreover, an approximate emergent-level description in terms of macroscopic variables can be derived from the microscopic rules, which we use to analyse the system's collective and long-term behaviour by applying tools from dynamical systems theory.

We discuss three adaptive-network models for different collective phenomena within our common framework. First, we propose a novel approach to collective motion in insect swarms, in which we consider the insects' adaptive interaction network instead of explicitly tracking their positions and velocities. We capture the experimentally observed onset of collective motion qualitatively in terms of a bifurcation in this non-spatial model. We find that three-body interactions are an essential ingredient for collective motion to emerge. Moreover, we show what minimal microscopic interaction rules determine whether the transition to collective motion is continuous or discontinuous.

Second, we consider a model of opinion formation in groups of individuals, where we focus on the effect of directed links in adaptive networks. Extending the adaptive voter model to directed networks, we find a novel fragmentation mechanism, by which the network breaks into distinct components of opposing agents. This fragmentation is mediated by the formation of self-stabilizing structures in the network, which do not occur in the undirected case. We find that they are related to degree correlations stemming from the interplay of link directionality and adaptive topological change.

Third, we discuss a model for the evolution of cooperation among self-interested agents, in which the adaptive nature of their interaction network gives rise to a novel dynamical mechanism promoting cooperation. We show that even full cooperation can be achieved asymptotically if the networks' adaptive response to the agents' dynamics is sufficiently fast. 



\section{Contents}

1. Introduction 1

2. Complex and adaptive networks 5

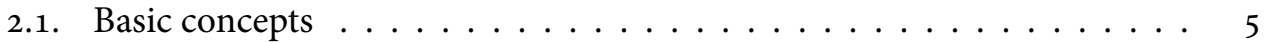

2.2. Network models . . . . . . . . . . . . . . . . . . . . . 8

2.3. Dynamical processes on networks . . . . . . . . . . . . 10

2.4. Adaptive networks . . . . . . . . . . . . . . . . . . . . 11

3. Simulation and analysis of adaptive networks 19

3.1. Rule-based modelling . . . . . . . . . . . . . . . . . . 19

3.2. Adaptive-network simulation . . . . . . . . . . . 23

3.3. Emergent-level description . . . . . . . . . . . . . . . 26

4. Swarming behaviour 37

4.1. Collective motion in swarms . . . . . . . . . . . . 37

4.2. Adaptive-network approach to swarming $\ldots \ldots \ldots \ldots \ldots \ldots$

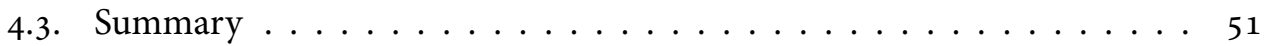

5. Opinion formation 53

5.1. The voter model . . . . . . . . . . . . . . . . . . 54

5.2. Early fragmentation in directed adaptive networks . . . . . . . . . 63

5.3. Summary . . . . . . . . . . . . . . . . 78

6. Evolution of cooperation $8 \mathbf{8 1}$

6.1. Cooperation in nature and society . . . . . . . . . . . . . 81

6.2. Evolutionary game theory $\ldots \ldots \ldots \ldots \ldots$

6.3. Mechanisms promoting cooperation . . . . . . . . . . . 88

6.4. Cooperation in adaptive networks . . . . . . . . . . . . . . 90

6.5. A homoclinic route to full cooperation . . . . . . . . . . . . . 94

6.6. Summary . . . . . . . . . . . . . . . . . . 103

7. Conclusions 105

List of abbreviations $\quad 109$

A. Surrogate rules for non-linear rates 
Contents

B. Bifurcations in dynamical systems

115

C. The replicator equation

123 


\section{Introduction}

Collective phenomena are ever-present in our day-to-day lives. This is irritatingly obvious in the usual traffic jam on our way to work every morning, but there are many more less annoying examples. Collective phenomena are bound to appear in systems built from a large number of interacting units, such as the cars during rush hour, football fans in a stadium performing the Mexican wave, or investment bankers whose collective behaviour determines the rise and fall of stock prices. In fact, one may be tempted to say that life is a collective phenomenon, driven by the collective dynamics of molecules, genes, cells, organisms, groups, societies, and so on.

Collective phenomena appear as a macroscopic effect resulting from the microscopic interactions of many units. While these microscopic interactions are in many cases well-understood, it is often unclear how they can give rise to unexpected, large-scale behaviour which is very different from the microscopic dynamics. The functioning of a car is perfectly well-known, and so is how to operate it if one wants to go faster or slower in order to avoid collisions with other cars. Yet, what triggers the sudden traffic jam in the so far smoothly flowing road traffic largely remains a mystery.

The relationship between the microscopic interactions of a system's constituting entities and its observed macroscopic behaviour has for long been a fundamental research question in a wide range of disciplines. In the social and economic sciences, it is known as the "micro-macro" paradigm (Schelling, 1978), and it is strongly connected to the concept of emergence and complexity in physics and philosophy (Anderson, 1972 Fromm 2004). Understanding the connection between the microscopic properties of a system and their macroscopic manifestation is one of the major goals in statistical physics. Many powerful concepts and tools have been developed in this field, with successful applications in physics, biology, and chemistry.

Within the framework of statistical physics, the aggregate phases of matter can be understood as collective phenomena, as well as the phase transitions from one to the other, or for instance, the phenomena of superconductivity and superfluidity. Moreover, the concepts and methodology of statistical physics are not restricted to the traditional areas of research, but have been exported also to seemingly disparate fields, such as neuroscience (Dayan and Abbott. 2005) and sociology (Castellano et al. 2009).

One of the central notions in statistical physics is the concept of emergence. Collective phenomena are emergent in a system of interacting units, which means that they cannot be anticipated from the microscopic properties of the units alone, but arise from the interactions among many of them (Anderson, 1972). Systems that display such emergence are often called complex systems, although many concurrent definitions exist for this term (e.g. Standish, 2008). One possible such definition characterizes a complex system as 
[...] a system in which large networks of components with no central control and simple rules of operation give rise to complex collective behaviour, sophisticated information processing, and adaptation via learning or evolution (Mitchell, 2009).

In this definition, an important concept for the understanding of complex systems is introduced: Although the nature of the interacting entities may differ fundamentally in different systems, they generally can be viewed as an abstract or physical network. In this network, the interacting parts of the system are represented by abstract nodes and the interactions among them by links, which may undergo complex changes over time. Many different systems can be considered from this point of view, so that the common feature of an underlying network structure may serve as a starting point for a unifying description of collective phenomena in complex systems. In sociology, for instance, the concept of a network has for long been used to represent the social relations among individuals, and it was one of the main insights in this field that there is an intimate coupling between the behaviour of individuals and the structure of their social network (Freeman, 2004 Borgatti et al. 2009.

Networks are, however, not merely useful representations of social relations, but provide a powerful abstraction of complex systems. By considering its network structure, one may condense the essential features of a complex system into a simplified model, where much of its complexity can be hidden in abstract nodes and links. A network model emphasizes the role of the interactions among the many parts forming a complex system instead of focusing on their specific internals, which are often rather complex by themselves. Such simple, often conceptual models provide fundamental insights about the basic principles in complex systems and allow to draw connections and analogies between different fields and applications. For this reason, the "network perspective" has been adopted in many research areas beyond traditional sociology. The properties and dynamics of networks themselves have recently become the focus of a dedicated research field termed "network science" (Börner et al. 2007).

Traditionally, the origin of certain network properties and their dynamical implications are largely considered separately in network science. More precisely, the processes generating particular network structures (the dynamics of the network) and the dynamical interactions of the nodes in a given network structure (the dynamics on the network) are investigated in separate lines of research. For most real-world networks, however, this separation appears rather arbitrary because their structure and dynamics typically evolve simultaneously. Moreover, the network structure often changes in response to the ongoing dynamics on the network, creating a feedback loop between the dynamics of the nodes and the evolution of their interaction topology. Networks containing such a feedback loop are called adaptive or coevolutionary networks (Gross and Blasius, 2008 Gross and Sayama, 2009).

The coupling between local and topological degrees of freedom can give rise to novel macroscopic phenomena in adaptive networks. For instance, complex, hierarchical net- 
work topologies can self-organize in adaptive networks, and distinct classes of nodes can emerge from an initially homogeneous configuration. Such phenomena are ubiquitous in natural adaptive networks, e.g. biological or chemical networks, and have been observed in a number of adaptive-network models (Gross and Blasius, 2008, and references therein). Understanding the dynamics of adaptive networks is a challenge to existing modelling approaches and analytical frameworks from network science. These approaches must be extended and new frameworks must be developed to address the dual nature of adaptive networks, in which both the network structure and the local node states evolve simultaneously.

In this thesis, I present a modelling framework for adaptive networks that extends existing approaches for the simulation and analytical description of non-adaptive networks. In this rule-based framework, an adaptive-network model is defined in terms of its microscopic processes, i.e. its local interaction rules, and a prescription of how these rules are applied to evolve the system. To study the model dynamics, the microscopic rules are directly implemented in agent-based simulations of the system. Moreover, I discuss how an approximate, macroscopic description of the emergent dynamics can be derived from the microscopic rules in terms of a low-dimensional system of differential equations. I show how the analysis of this coarse-grained analytical description using the tools from dynamical systems theory helps understand the global behaviour of adaptive-network models.

In the main part of this thesis, I propose three different adaptive-network models for collective phenomena in three different research fields, namely collective motion in animal groups, opinion formation in social networks, and the evolution of cooperation in evolutionary game theory. Considering the different systems studied in these fields as adaptive networks makes it possible to investigate their dynamics within the same framework and reveal their similarities.

This thesis is organized as follows: in chapter 2, I give a brief overview of the basic concepts from graph theory and network science used in this work. I introduce adaptive networks, showing their ubiquity in natural and man-made systems, and review the recent research on adaptive networks.

In chapter 3, the modelling framework is introduced. It is based on the concepts of a transformation rule, which defines a microscopic process or interaction, and an update scheme, which determines how to apply a set of transformation rules. I discuss the specifics of implementing efficient agent-based simulations of large adaptive networks. Further, I demonstrate how an emergent-level description can be derived from the transformation rules by considering coarse-grained, macroscopic variables and using suitable approximations.

The modelling framework for adaptive networks is applied to the phenomenon of collective motion in insect swarms in chapter 4 . We propose an adaptive-network model for a swarming experiment that studied collective motion in desert locusts. Inspired by models of opinion formation in groups of interacting individuals, our model only considers 


\section{Introduction}

the insect's marching directions as node states, which can change due to interactions, and neglects all spatial embedding. Although being a highly simplified, non-spatial model, it reproduces qualitatively the experimentally observed onset of collective motion in terms of a phase transition. Using our framework, we can address the questions what minimal interaction rules lead to collective behaviour, and which microscopic interactions determine whether collective motion sets in in a continuous or discontinuous transition. In chapter 5 , we further consider the theme of opinion formation in groups of individuals and propose an extension of the well-known voter model to directed adaptive networks, in which we take the asymmetric character of social relationships into account. In particular, we are interested in the effect of directed links in adaptive networks. We show that the fragmentation transition in the adaptive voter model, in which the social network breaks into distinct components of opposing agents, is accelerated in directed networks by a novel mechanism, which we call early fragmentation.

In chapter 6, we consider the evolution of cooperation in groups of self-interested individuals. We ask how the adaptive nature of their social interactions can promote cooperative behaviour even though all individuals act in their own interest only. We propose an adaptive-network model for the evolution of cooperation in a population of selfish agents, whose interactions are modelled in the framework of evolutionary game theory. Here, we observe a novel mechanism that promotes cooperation dynamically and opens a new route to almost full cooperation. This mechanism relies on the coupling of topological and local dynamics in the adaptive network and can be understood from the emergent-level description of the system.

Finally, I summarize our findings in chapter 7 comparing the different models and synthesizing our results. Moreover, I discuss possible extension of our modelling framework and suggest directions for future work. 


\section{Complex and adaptive networks}

In the recent past, the study of networks and dynamical processes on them has received considerable interest across disciplines. Networks can be found in many different areas, ranging from biological networks, such as protein interaction networks, gene-regulatory networks, food webs, etc., to social and technological networks, such as friendship and acquaintance networks, or the Internet (Barrat et al., 2008; Boccaletti et al., 2010). The increasing availability of large datasets on online (social) networks has boosted research on topics as diverse as epidemic spreading, information dissemination, opinion and community formation, attack resilience, or efficient routing in networks. Concerned with the analysis and modelling of complex networks from diverse complementary perspectives, network science brings together different research questions, applications, and approaches from a wide range of disciplines, such as biology, sociology, physics, engineering, mathematics, and computer science (Barabási, 2002, Watts 2003 Börner et al. 2007).

Both the quantitative analysis of complex networks and the understanding of their basic mechanisms have greatly benefited from the methodology of mathematics and statistical physics. In the next section I will give a brief overview of the basic concepts of the 'statistical mechanics of complex networks' (Albert and Barabási 2002), which are most relevant for the purpose of the present work. A detailed introduction in the analysis and modelling of networks can be found in (Newman, 2003, Newman et al. 2006; Newman. 2010).

\subsection{Basic concepts}

A network is an abstract representation of a set of entities and the relations that exist among them. It consists of a number of nodes connected by links. Mathematically speaking, a network is a graph $G(V, E)$ formed by the set $V$ of nodes $i \in V$ (also called vertices) and the set $E$ of links $(i, j) \in E$ (also called edges). As such, it is a well-studied object in the venerable mathematical discipline of graph theory, which has its origins in Euler's work on the Königsberg bridge problem (Euler. 1736. Bollobás, 1998 , Diestel, 2005).

In many networks, the links represent unidirectional relations, such as the links between websites, or the regulatory relations between genes or proteins (Fig.2.1). In this case, the network is directed, and the notation $(i, j) \in E$ means that there exists a link from node $i$ to node $j$ (but not necessarily from $j$ to $i$ ). I will occasionally also write $i \rightarrow j$ to denote a directed link. By contrast, in undirected networks, no specific direction is assigned to the links. Undirected networks can be viewed as directed ones in which the existence of a 

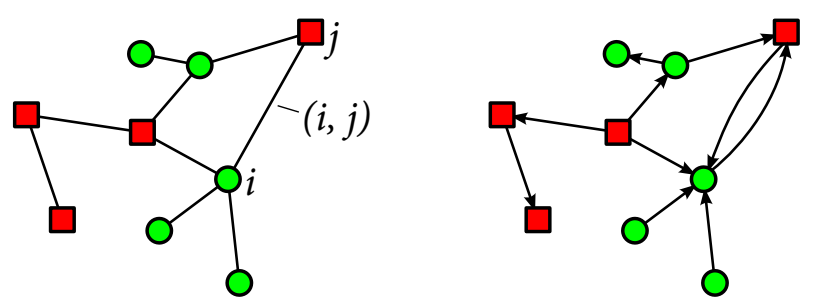

Figure 2.1.: Networks with two different node types/states. Two nodes $i$ and $j$ are marked, as well as the connecting link $(i, j)$. Left: undirected network. Right: directed network with a pair of reciprocal links.

link $(i, j) \in E$ always implies that there also exists $(j, i) \in E$.

Both the nodes and links can be associated with additional variables, such as node states or link weights. For instance, the nodes could be assigned one of two labels $s \in\left\{0^{7}\right.$, $\left.\&\right\}$ representing men and women in a social network, and the links could represent friendship, acquaintance, or sexual relationship. In this example, a "weight" could be assigned to each link, i.e. a scalar variable measuring the intensity of the respective relation.

\section{Degree distribution}

The basic topological property in networks is the degree of a node, defined as the number of neighbours the node is connected to. The node degrees in a network (or an ensemble thereof) are distributed according to the degree distribution $p_{k}$, which gives the probability that a random node has exactly $k$ neighbours. If the network is directed, a node has two different degrees: an in-degree counting the number of incoming links and an outdegree counting the number of outgoing links. In this case the probability of finding a random node with exactly $k_{\text {in }}$ incoming and $k_{\text {out }}$ outgoing links is given by the joint distribution $p\left(k_{\text {in }}, k_{\text {out }}\right)$. From this distribution, the in- and the out-degree distributions can be computed as the respective marginal distributions $p_{\text {in }}(k)=\sum_{k^{\prime}} p\left(k, k^{\prime}\right)$ and $p_{\text {out }}(k)=\sum_{k^{\prime}} p\left(k^{\prime}, k\right)$.

The degree distribution offers a simple means of identifying different classes of networks. In degree-homogeneous networks such as regular lattices, every node has the same degree $k_{0}$ and $p_{k}=\delta_{k k_{0}}$. Moderately heterogeneous undirected networks of relatively small variance in the node degrees are conveniently characterized by their mean degree $\langle k\rangle=2 K / N$, where $N$ is the number of nodes and $K$ is the number of links in the network. Many real-world networks, however, have strongly heterogeneous degrees following exponential, power-law, or mixed distributions. For instance, the Internet appears to have degree distributions ${ }^{11} p_{k} \propto k^{-\gamma}$ for large $k$, with $2 \leq \gamma \leq 3$ (Broder, 2000),

\footnotetext{
${ }^{1}$ The Internet can be viewed as a (directed) network on different levels. Typically, one considers the "autonomous systems" level or the level of "class C subnets". On a more fine-grained scale, the WorldWide Web is considered, which is the network formed by the set of all websites.
} 
although this result has been subject to some debate, as it seems to depend on the way of measuring (Chen et al. 2002).

Networks that show such a power-law degree distribution with $2 \leq \gamma \leq 3$ are called scale-free, because they do not possess a characteristic scale or typical degree. For large scale-free networks, the second moment of the degree distribution diverges quickly, so that the mean degree computed from $p_{k}$ becomes meaningless. In particular, both the mean degree and the second moment tend to infinity for $\gamma=2$ in the limit of infinite network size. As it turns out, the degree distributions of many real-world networks can be fitted by a scale-free distribution (Barabási and Albert, 1999, Albert and Barabási, 2002. Newman 2010). The quality and significance of such fits, however, have been subject to criticism (Stumpf et al. 2005: Clauset et al. 2009).

In the analysis of networks and their dynamics, it is often necessary to consider another type of degree distribution, which is derived from $p_{k}$. In contrast to the degree distribution $p_{k}$, which is the probability to select a node of degree $k$ by picking a random node from the network, the distribution $g_{k}$ is the probability to select a node of degree $k$ by following a random link. Picking a random link from the network and choosing one of the nodes it connects, one has the probability $g_{k}$ that this node has exactly $k$ neighbours. As a node with degree $k$ can be selected in this way via any of its $k$ links, the probability $g_{k}$ must be proportional to $k p_{k}$, and hence $g_{k}=k p_{k} /\langle k\rangle$ is the properly normalized distribution. A variant of this distribution, the excess degree distribution (Newman 2003), is also often used: $q_{k}=g_{k+1}$ is the probability that a node selected by following a random link has exactly $k$ additional links apart from the one by which it has been reached. The expected number of additional links of a node selected in this way is hence given by the mean excess degree $\langle q\rangle=\sum_{k} k q_{k}=\left\langle k^{2}\right\rangle /\langle k\rangle-1$, where $\left\langle k^{2}\right\rangle$ is the second moment of the degree distribution $p_{k}$.

\section{Average path length}

The distance between two nodes is defined as the number of links in the shortest path from one to the other (if there is any). The average of all distances between any two nodes in the network is called its average path length $\ell$. For degree-homogeneous networks, such as regular $d$-dimensional lattices, this quantity scales with the system size $N$ as $\ell \propto N^{1 / d}$. By contrast, real-world networks typically have relatively small average path lengths scaling as $\ell \propto \ln N$ (Albert and Barabási, 2002). The relative compactness of real-world networks resulting from this slow scaling is known as the "small-world" effect (Watts and Strogatz 1998) or the famous notion of "six degrees of separation", which refers to the surprisingly low average path lengths in social acquaintance networks (Milgram. 1967. Watts 2003).

Slow scaling of the average path length does not, however, imply a particular organization principle. It is found in a wide range of network classes, including totally random networks obtained by randomly placing links among a given number of nodes, which will be introduced in 2.2 


\section{Complex and adaptive networks}

\section{Clustering}

A common feature observed in real-world networks is clustering. In social networks, for instance, two friends of someone are often also friends with each other. Cliques of friends are a typical extreme example, in which everybody is connected to everybody else. This locally high interconnectivity can be quantified by the clustering coefficient. For any node $i$ in the network, it is defined as $C_{i}=2 E_{i} / k_{i}\left(k_{i}-1\right)$, where $k_{i}$ is the degree of the node, and $E_{i}$ is the total number of links among the neighbours of $i$. Thus, $C_{i}$ measures the local degree of clustering as the ratio of the number of realized connections among $i$ 's neighbours and the number of possible connections among them, which is given by $k_{i}\left(k_{i}-1\right) / 2$. As a global measure of clustering in the network, one uses the average clustering coefficient $C=\sum_{i} C_{i} / N$. As an alternative measure, one sometimes uses the transitivity $C^{\prime}=3 \Delta / T$, where $\Delta$ is the total number of closed triangles in the network, and $T$ is the total number of connected triples, i.e. three nodes connected in a line or a triangle (Watts and Strogatz. 1998). This measure is also called the "fraction of transitive triples" in the sociology literature (Wasserman and Faust, 1994). Most real-world networks exhibit a significant amount of clustering, with average clustering coefficients of about $C \approx 0.1 \ldots 0.8$ (Albert and Barabási, 2002).

\subsection{Network models}

In order to understand the origin and the structure of networks, many simple models of network formation have been proposed. They are typically formulated as constructive algorithms, which sample from an ensemble of networks satisfying certain constraints (Bollobás 2001). Network formation models aim at reproducing the structural features observed in real-world networks. In the first paradigmatic models, the most important such features were scale-free degree distributions, small average path lengths, and strong clustering. I will briefly introduce the three most prominent network formation models in this section.

\section{Erdős-Rényi random graphs}

The simplest model of network formation is the Erdős-Rényi (ER) model of random graphs (Erdös and Rényi, 1959, 1960). Starting from $N$ nodes, it generates a random undirected network. It samples uniformly from the ensemble of networks with exactly $N$ nodes and $K$ links, which is achieved by connecting at random $K$ pairs of nodes among the $N(N-1) / 2$ possible ones. The random graph ensemble with exactly $N$ nodes and $K$ links, or an instance thereof, is sometimes denoted as $\mathcal{G}(N, K)$. A variant of the original ER model is the ensemble $\mathcal{G}(N, p)$, which is considerably easier to analyse mathematically. In this variant, each of the $N(N-1) / 2$ possible links is realized with the fixed probability 
$p$. The degree distribution of the resulting network is a binomial distribution,

$$
p_{k}=\left(\begin{array}{c}
N-1 \\
k
\end{array}\right) p^{k}(1-p)^{N-1-k}
$$

with mean degree $\langle k\rangle=p(N-1)$. In the thermodynamic limit of large $N$ with fixed $\langle k\rangle$, it converges to the Poisson distribution,

$$
p_{k}=e^{-\langle k\rangle} \frac{\langle k\rangle^{k}}{k !}
$$

which is why networks from the ensemble $\mathcal{G}(N, p)$ are also called Poisson networks.

The simplicity of the ensemble $\mathcal{G}(N, p)$ makes it possible to compute analytically most of its properties. For this reason, it is an attractive baseline model for various applications, although it does not reproduce typical features of real-world networks such as a scale-free degree distribution or strong clustering. In an ER graph, the average path length scales as $\ell \propto \ln N / \ln \langle k\rangle$, while the average clustering coefficient is $C \propto\langle k\rangle / N$ due to the independent placement of links (Newman et al. 2001; Albert and Barabási. 2002). This means that large ER graphs exhibit very small clustering, so that the local neighbourhood of any node is expected to be tree-like, i.e. clear of closed triangles or loops. In fact, the probability of finding a closed triangle in a given ER graph is $\propto 1 / N$.

It is worth noting that for networks with a Poisson degree distribution, such as the ER networks, the excess degree distribution $q_{k}$ is also Poissonian. In particular, $\langle q\rangle=\langle k\rangle$ in Poisson networks.

\section{Watts-Strogatz small-world networks}

Although the ER model reproduces the small-world effect, i.e. an average path length $\ell \propto \ln N$, it fails to capture the observed strong clustering in real networks. A network formation model that generates both short path lengths and strong clustering is the WattsStrogatz (WS) model (Watts and Strogatz. 1998). In the literature on complex networks, the term "small-world network" is often used synonymously with the WS model.

Starting from an already clustered network, the average path length is reduced by rewiring a fraction of the links to create long-range connections. In the original WS model, $N$ nodes are initially connected in a ring, so that each node is connected to its $k$ closest neighbours in this ring (with $k$ even). Then, for each link, one of the link ends is rewired to a random node with probability $p$, avoiding self-loops and multiple links between the same two nodes. A fraction $p$ of the $k N / 2$ links is thus converted to longrange links, which leads to a fast decrease in the average path length, while the average clustering decreases much slower. Tuning the parameter $p \in[0,1]$, one can interpolate between a regular ring graph and a random network. 


\section{Complex and adaptive networks}

\section{Preferential attachment}

By construction, both the ER and the WS model produce networks with narrow degree distributions. To generate broad degree distributions, a different approach is required. The most popular network evolution model generating scale-free networks is the preferential attachment algorithm. Originally introduced as a model for citation networks in scientific publications (de Solla Price, 1965), its roots date back to statistical models for word frequency distributions (Yule 1944: Simon 1955). It leapt into fame when Barabási and Albert (1999) realized its potential relevance for many different real-world networks. For this reason, the preferential attachment algorithm is usually referred to as the BarabásiAlbert (BA model.

The BA model combines two ingredients to produce scale-free networks: network growth and preferential attachment. Starting from a small seed graph of $m_{0}$ nodes, new nodes are added continuously to the network. Every new node is connected to $m \leq m_{0}$ existing nodes such that the probability $\Pi_{j}$ of connecting to an already present node $j$ is proportional to the degree $k_{j}$ of this node, namely $\Pi_{j}=k_{j} / \sum_{i} k_{i}$. It is straightforward to compute the asymptotic degree distribution this algorithm generates (see, e.g., Albert and Barabási, 2002). In the limit of large networks it approaches $p_{k} \propto k^{-3}$.

The power-law degree distribution implies that there exist a few high-degree nodes, which connect to a large fraction of the network, whereas most of the nodes connect to only a few others. These high-degree "hub" nodes efficiently connect different parts of the network, which is why scale-free networks have short average path lengths. In the $B A$ model, the average path length scales with the number of nodes as $\ell \propto \ln N / \ln \ln N$, and the clustering coefficient approximately follows a power law $C \propto N^{-\alpha}$ with $0<\alpha<1$ (Bollobás, 2001).

The combination of growth and preferential attachment has become the paradigmatic mechanism that generates a scale-free degree distribution. It provides a plausible scenario for the emergence of scale-free degree distributions in many real-world networks. However, scale-free degree distributions can also be generated by a number of different models, some of which also produce significant clustering and/or small average path lengths (Caldarelli et al. 2002, Holme and Kim, 2002, Klemm and Eguíluz, 2002a b Gómez-Gardeñes and Moreno, 2006). Therefore, preferential attachment is only one of several known mechanisms that might have shaped the degree distributions of real-world networks (cf. Keller, 2005).

\subsection{Dynamical processes on networks}

The network formation models discussed above aim at generating specific topologies that reproduce observed features of real-world networks. Using these models to produce wellcontrollable network topologies, one can investigate how the structure of the interaction network or certain network properties affect the dynamics of the constituting elements, i.e. the nodes and links. In this approach, the network structure is considered as the 
underlying interaction geometry for some dynamical process. It is a straightforward extension to the established practice of studying dynamical processes on regular lattices of some dimension. For instance, the Ising model of ferromagnetism has been studied on networks of different degree distributions, revealing that its critical temperature and the order of the phase transition depend strongly on the underlying network structure (e.g.) Dorogovtsev et al. 2002, 2008).

Many dynamical processes can be studied on networks (Barrat et al. 2008). In general, a node's state is described by one or more dynamical variables, whose evolution is determined by some internal dynamics of the node and the coupling to its network neighbours. A simple example is the propagation of a disease in a contact network of susceptible agents (Liljeros et al., 2001, Keeling and Eames, 2005). In this case, the node states are binary variables: a node is either healthy and hence susceptible to the disease, or it has been infected and is thus infectious itself. A susceptible node may contract the disease from an infectious neighbour, become infectious itself, and pass the disease on to the next susceptible neighbour. Thus, the contact network in which the nodes are connected provides the geometry in which the disease can spread. Depending on the network topology and the infectiousness of the disease, an epidemic may occur or the disease may die out (Boots and Sasaki, 1999: Kuperman and Abramson, 2001, Pastor-Satorras and Vespignani, 2001a).

Further examples of dynamical processes on networks include synchronization phenomena and resource flows or diffusion in networks. In simple conceptual models of, e.g., neuronal networks, the synchronization of oscillators coupled through an interaction network is considered (Kuramoto, 2003, Moreno and Pacheco, 2004, and references therein). The flow of resources is of interest in models of diffusion on networks (Noh and Rieger 2004), traffic flows in road networks and transport of data or information in technological networks (Barthélemy and Flammini. 2006. Tadić et al., 2004), or food webs, which model the flow of biomass among predator and prey species (Pimm, 2002. Camacho et al., 2002. Gross et al. 2009). What most modelling approaches for these systems have in common is that they consider a fixed, static network topology providing the geometry in which the node dynamics takes place. Most real-world networks are, however, not static but changing continuously. Moreover, they may change in response to the dynamics on the network, thus establishing a coupling between the node dynamics and the topological change. Networks containing such a coupling are called adaptive networks.

\subsection{Adaptive networks}

Traditionally, the research on complex networks has focused on two key aspects of network dynamics. The first aspect is the emergence and evolution of particular network structures, that is to say, the dynamics of the network topology itself. Here, the mechanisms giving rise to observed properties of real-world networks are investigated, such as scale-free 


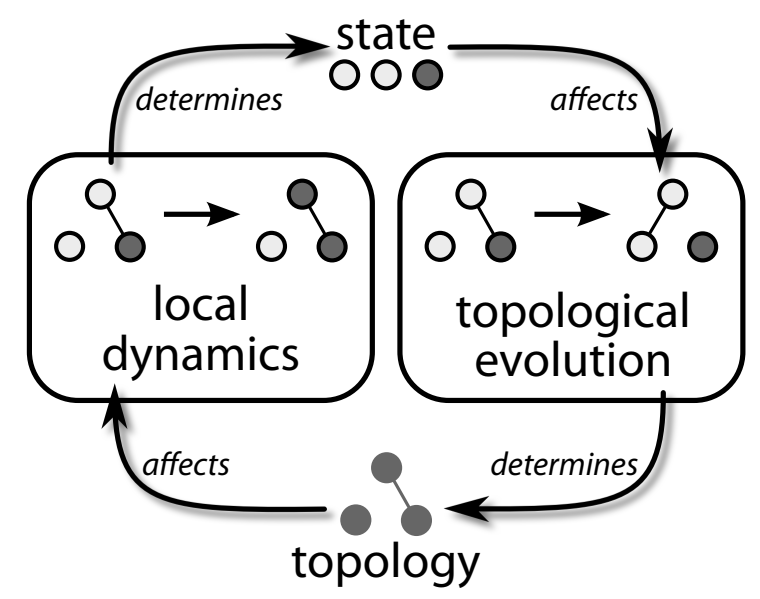

Figure 2.2.: In adaptive networks, the node dynamics affects the topological evolution, thus creating a feedback loop between the local dynamics on the network and the topological evolution of the network. Figure adapted from (Gross and Blasius, 2008).

degree distributions, short average path lengths, or strong clustering. To that end, simple network formation models have been designed, as for instance the $\mathrm{ER}$, the $\mathrm{WS}$ and the $\mathrm{BA}$ model introduced above.

The second aspect in the focus of complex network research is the dynamics of processes on the network. In this context, the network structure is considered as a static substrate on which some dynamical process takes place, such as disease propagation or message passing. In this case the network nodes are the dynamical systems of interest, whose interaction topology is determined by the network, as for instance in a network of coupled oscillators. The effect of the underlying topology is then investigated using different model networks as a substrate.

In most real-world systems, however, the underlying networks do not only provide a static interaction topology for some superimposed process, but rather evolve and change in time themselves. Moreover, the ongoing dynamical processes within the network can influence the topological change, providing a feedback loop between the dynamics of the network and the dynamics on the network (Fig. 2.2). That is to say, the dynamics of the nodes influence the network topology, while the network topology in turn influences the dynamics of the nodes. Networks in which such a feedback loop is present are called adaptive or coevolutionary networks (Gross and Blasius, 2008).

Many natural and man-made networks are adaptive networks. Distribution networks such as the vascular system, river networks, power grids, or the Internet are prime examples. In a distribution network, the nodes and links are subject to a certain load or throughput of resources, i.e. blood, water, power, or data. If the load on a given link exceeds its capacity, the link fails, leading to congestion or even breaking the link, so that it is effectively removed from the network. The same is true if the load on a node exceeds 
its capacity. On a longer time scale, however, the network may respond adaptively to persistent high load by reinforcing the affected parts and/or creating new connections that take part of the load. During the thawing period, for instance, many small creeks are added to the network of rivers, because the existing rivers cannot take all the load from the increased inflow, bursting their banks. Within geological time scales, however, these small creeks can shape the landscape and become rivers themselves, thus providing new paths in the network that relieve the high load. Similarly, very busy roads are often congested with traffic jams, leading to their short-term failure in the traffic network, but also leading to a possible long-term expansion (after an even more congestion-prone period of road works, of course).

Further examples are found in neural networks, which are also highly adaptive, strengthening or weakening the synaptic connections in response to the activity of the neurons. Moreover, most social networks are adaptive networks, in which connections are formed, for instance, between like-minded individuals, and opinions are adjusted to the opinions of social contacts. For an overview of these and other examples, see Gross and Blasius (2008), Blasius and Gross (2009), Gross and Sayama (2009), and references therein.

Dynamical processes taking place on a (fixed) network are in general highly sensitive to the underlying topology. The network structure is thus reflected in the local dynamics, and in some sense the dynamics encodes topological information (Gross and Sayama. 2009). Indeed, this mapping of the network topology to the local dynamics may be used to identify certain topological features of the network by analysing the behaviour of dynamical processes on the network (Reichardt and Bornholdt, 2004, Arenas et al. 2006. Hu et al. 2008).

Global topological information is thus made locally accessible to some extent, as it determines the local dynamics of the nodes. In an adaptive network, the nodes can use this global information in making local changes to the topology, which will then be reflected again in the dynamics. This intricate feedback loop in adaptive networks gives rise to genuinely novel phenomena. Gross and Blasius (2008) observed that several phenomena appear as a common theme in many different adaptive networks. They proposed four (non-exhaustive) "hallmarks" of adaptive-network behaviour, namely robust dynamical self-organization (often towards critical behaviour), spontaneous division of labour, the formation of complex topologies, and complex system-level dynamics. In the following, I will briefly discuss these phenomena and provide some examples, closely following and summarizing the review in (Gross and Blasius, 2008).

\section{Robust self-organization}

The feedback of global information into the topological evolution through the local dynamics can allow an adaptive network to self-organize towards a critical state. This is well illustrated in the example of Boolean threshold networks, which are simple models for gene regulatory and neural networks (Kauffman, 1969: Derrida et al. 1987). In these models, a node's state is represented by a single Boolean variable that encodes whether 


\section{Complex and adaptive networks}

a certain gene is being transcribed or a neuron is firing. A node becomes active only if the inputs it receives through its links exceed a certain threshold, where the links connecting the nodes represent promoting or inhibiting interactions. Bornholdt and Rohlf (2000) considered an adaptive threshold network, in which the local dynamics are complemented by a topological update rule. This rule is based on monitoring random nodes for a fixed time, during which the system is moving on an attractor or chaotically. Then, new connections are established to "frozen" nodes, i.e. nodes that do not change their state within this fixed time. Conversely, existing connections are removed from "dynamical" nodes, which flip their state at least once while monitored.

The topological update rule thus changes the average degree in the network. In their model, Bornholdt and Rohlf found that an average degree of $\langle k\rangle=2$ is approached in the limit of infinite networks independently of the initial conditions. This is closely related to a topological phase transition which can be observed in the non-adaptive variant of this model, where no links among the nodes are created or deleted. When varying the average degree as a parameter, the average fraction of frozen nodes in the network drops from 1 to 0 at a critical value $\langle k\rangle_{c}=2$. In the adaptive network, the topological update rule therefore almost always adds links if $\langle k\rangle<2$, because almost all nodes are frozen in this case, whereas it almost always deletes links if $\langle k\rangle>2$. Hence, the average degree tends to increase if it is below, and to decrease if it is above $\langle k\rangle_{c}$. The network thus self-organizes towards the critical average degree $\langle k\rangle_{c}=2$.

This model is an example of how global information is made locally accessible by the dynamics and used in local topological update rules: The global fraction of frozen nodes is sampled stochastically by locally monitoring random nodes for activity. This global information is then used in the local topological rule that creates or deletes links accordingly. Thus, a global order parameter associated to a phase transition becomes locally accessible and can be used to self-organize the system towards the phase transition.

This dynamical self-organization is highly robust. It does not depend sensitively on the initial conditions nor the specific parameter choice. It was also observed in further studies on different models (Christensen et al. 1998, Bornholdt and Röhl, 2003, Liu and Bassler, 2006), including a somewhat realistic model of spike-time-dependent plasticity in neural networks (Meisel and Gross 2009), which highlights the generality of the underlying mechanism.

\section{Spontaneous division of labour}

The co-evolution of the node states and the network topology in adaptive networks can lead to the emergence of distinct classes of nodes from an initially homogeneous population. This spontaneous "division of labour" was demonstrated in an adaptive network of coupled oscillators by Ito and Kaneko (2001). In their model, a number of onedimensional chaotic oscillators is coupled in a weighted directed network. The node states evolve according to a logistic map, which depends on the states of the network neighbours. The network topology is changed according to an update rule that redistributes the link 
weights: link weights between oscillators whose difference in the state variables is not too large are increased, while the total weight of all incoming links of each node is kept constant.

For certain parameters, the nodes differentiate into two distinct classes that differ in their effective out-degree, namely a class of nodes with a high total weight of their outgoing connections, and a class of nodes with a low total weight of their outgoing connections. In the distribution of in-degrees, however, no difference between the two classes is observed. From an initially homogeneous population, the two degree classes emerge and remain largely separated once they have formed, even though the system does not reach a frozen configuration. That is to say, a node in the low-degree class generally remains in the low-degree class for a long time, and the same is true for the high-degree nodes. As the nodes in the network are coupled so that each node is influenced by its incoming connections, a node with a high effective out-degree is an influential node that has an impact on many others. By contrast, a low-degree node only influences a small number of other nodes while still receiving a similar number of inputs as a high-degree node. In this sense, Gross and Blasius (2008) described the emergence of the two classes as the emergence of "leaders" and "followers" in the system, or as a spontaneous "division of labour."

A similar emergence of different node classes has been observed in related models, in which the topological evolution is generally such that connections are strengthened between nodes in a similar state (Gong and van Leeuwen, 2004 van den Berg and van Leeuwen 2004). Emergent "leadership" has also been found in a game-theoretic model of the Prisoner's Dilemma in an adaptive network (Zimmermann et al. 2004, Eguíluz et al. 2005), where the nodes imitate other nodes in self-organized hierarchical structures.

\section{Formation of complex topologies}

The emergence of leaders and followers is one example of how heterogeneous structures can be formed from homogeneous initial conditions by the feedback of the local dynamics on the topological evolution in adaptive networks. This feedback can achieve further, highly complex topologies, which has been observed in different models of opinion formation, evolutionary game theory, and epidemiology, and also in some of the new models discussed in the present work.

A prominent example of topological restructuring that can be achieved in adaptive networks is the emergence and separation of distinct communities in models of opinion formation. In contrast to the spontaneous division of labour in the model of Ito and Kaneko, where the network remains largely connected, network components may also become fully disconnected from each other as a result of adaptive rewiring rules. This is the case, for instance, in the voter model on adaptive networks, a paradigmatic model of opinion formation (Holme and Newman 2006: Vazquez et al. 2008). In this model, the nodes represent agents holding one of many possible opinions. A node can discard the link to an opposing neighbour (holding a different opinion) and establish a new 


\section{Complex and adaptive networks}

connection to a random consenting node (holding the same opinion as the focal node). Further, a node can change its state by adopting the opinion of a neighbour. In this simple model, a generic phase transition occurs at a critical value of the time scale ratio between rewiring dynamics and opinion adoption. Below the transition, the topological change is slow compared to the node state dynamics, allowing the system to reach a state of full consensus, in which all nodes have the same opinion. Above the transition point, however, the fast rewiring dynamics leads to the separation of the randomly distributed initial opinions, and the network splits into many disconnected components reflecting the initial conditions. Each of these components is internally in consensus, i.e. all nodes in one component have the same opinion. Similar group segregation phenomena have been observed in related epidemiological and game-theoretic models (Gross et al. 2006. Gräser et al. 2009).

The emergent structure of disconnected "consensus communities" is a global property of the network topology, which is achieved by processes acting on the local neighbourhood of the nodes. In adaptive networks, this is possible because the topological change is driven by the node dynamics. Thus, global structure can emerge from local rules in adaptive networks.

The emergence of global structure from local interactions has also been demonstrated in a recent game-theoretic model of cooperation in weighted adaptive networks (Do et al. 2010). In this model, the nodes represent agents making investments in the interactions with other agents, such as firms investing in collaborations with other firms, for instance. The links represent these interactions, with the associated link weights representing the investments of each partner. The benefit obtained from an interaction depends on the investment made by the interacting partners. By increasing or decreasing their investments in different interactions, the nodes try to optimize the total benefit they obtain.

Under very general conditions, complex, stable interaction topologies are formed in this model. In these stationary configurations, some nodes have high degree, thus obtaining high benefits, and some nodes have only a single link, thus investing in a single interaction only. This pattern is similar to the emerging leadership discussed before. At the same time, bidirectionally connected communities arise, within which every node makes the same total investment and the total link weight of each link is the same. This strong coordination among individual nodes is achieved by purely local rules: although none of the nodes has enough information to assess the total investment made by any of its neighbours, all nodes settle on the same total investment.

Further examples of how complex topologies emerge in adaptive networks include the formation of hierarchical networks in different game-theoretic models of competition, where a scale-free degree distribution is achieved by local interaction rules (Zimmermann et al. 2000, Ebel and Bornholdt, 2002, Ren et al. 2006, Poncela et al., 2008). 


\section{Complex system-level dynamics}

In many adaptive networks, the dynamical feedback loop between the node states and the topology allows for new dynamical phenomena, which are not found in the corresponding non-adaptive systems. Novel types of phase transitions and bifurcations involving both local and topological degrees of freedom can be observed. In simple epidemiological models on adaptive networks, for instance, the coupling of local and topological dynamics creates a rich array of dynamical regimes. This has been shown in an adaptive-network model of disease propagation, in which the nodes represent healthy or infectious individuals, and the healthy nodes can contract the disease via the links to infectious ones (Gross et al., 2006, Gross and Kevrekidis, 2008). In addition to that, the healthy nodes rewire their links away from infectious neighbours to other, randomly selected healthy nodes at a certain rate.

In this model, different long-term dynamics are observed. As in non-adaptive networks, the disease only persists in the network if the rate of infection exceeds a certain threshold, otherwise it dies out. A non-zero rewiring rate increases this threshold, because it effectively reduces the number of links between healthy and infectious nodes. Further varying the rates of infection and rewiring reveals a bistable regime, in which both the endemic and the disease-free state are observed for different initial conditions, and an oscillatory regime, in which the number of infected nodes changes periodically.

As a further example, the fragmentation into disconnected parts in the adaptive voter model introduced above and similar models of opinion formation is the result of a dynamical phase transition made possible by the state-dependent rewiring of links (Gil) and Zanette, 2006, Kimura and Hayakawa, 2008, Böhme and Gross, 2011). Network adaptivity has also been found to produce phase transitions in many different models from game-theory (Pacheco et al. 2006b, Santos et al. 2006a: Szolnoki and Perc, 2009b.

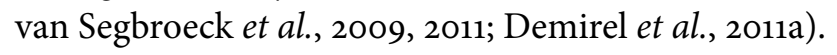





\section{Simulation and analysis of adaptive networks}

In this chapter, I introduce the formal framework used to define and analyse adaptivenetwork models in the present work. In this framework, a model is defined in terms of a set of microscopic transformation rules and an update mechanism, which together determine the evolution of an initial network configuration. I discuss how the emerging population-level dynamics observed in, e.g., computer simulations can be analysed using an approximate macroscopic description in terms of a low-dimensional system of ordinary differential equations.

\subsection{Rule-based modelling}

In the present work, I consider adaptive-network models whose dynamics can be specified in terms of simple, discrete, and local rules. In these models, each network node $i$ is associated with a discrete variable $\sigma_{i}$ taking values from a finite set $\mathcal{S}$, which describes the node's state or type. For instance, $\mathcal{S}_{2}=\{0,1\}$ could be used to describe inactive and active nodes representing deactivated and activated genes in a simplified gene regulation network. The same set may also refer to healthy and infected nodes in the spreading of a disease, or the two possible spin directions in an Ising model of ferromagnetism. The elements in the set $\mathcal{S}$ are usually labelled using upper-case Latin letters.

In the following, I introduce a framework by which the dynamics of such discrete adaptive networks can be described and analysed. The basic concepts of this framework are best explained building on an analogy with chemical reaction kinetics. On a macroscopic scale, chemical processes appear to run smoothly and in a continuous fashion. We know, however, that they consist of many distinct reaction events on a microscopic scale. Similarly, we can imagine the time evolution of a network to consist of many independent update events that change the network locally in some small neighbourhood. From this point of view, an adaptive-network model is defined by the node state set $\mathcal{S}$, the possible "reactions" that can occur, and their associated reaction rates. In the context of adaptive networks, I will refer to the microscopic reactions by the term transformation rules.

\subsubsection{Transformation rules}

The possible transitions in the local neighbourhood of a node are defined by stochastic transformation rules that depend on the node's state and the states of its neighbours. In their simplest form, these rules can be written symbolically as chemical reactions among the network units, such as nodes, links, or small subgraphs. They are associated with 
(a)

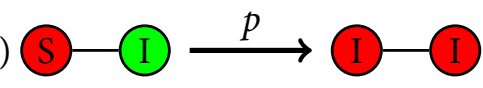

(c)

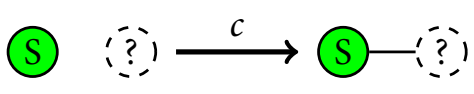

(b)

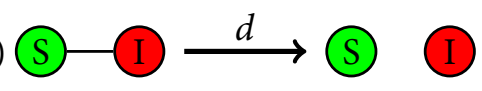

(d)

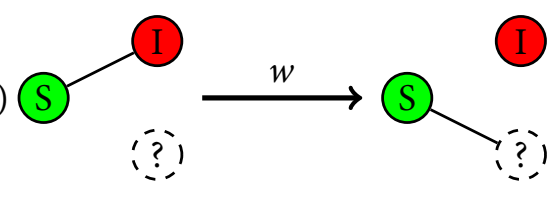

Figure 3.1.: Examples of different transformation rules: (a) contact process at constant rate $p$ per S-I-link, e.g., disease propagation from infectious (I) to susceptible (S) node; (b) link removal at rate $d$ per S-I-link; (c) link creation at rate $c$ per S node; (d) link rewiring at rate $w$ per S-I-link. A dashed circle denotes a random node picked from the whole population.

certain reaction rates, which can be simple constants or more elaborate functions. A transformation rule may locally change the nodes' states and/or the network topology. For the purpose of the present work, I consider only transformation rules that affect either the state of a node or the network topology, but not both at the same time (Fig. 3.1).

As an example of a rule affecting the state of a node, consider the so-called contact process $(\mathrm{CP})$. This prototypical process describes the transmission of a node's state to a neighbour via their connecting link, such as the propagation of a disease from an infected node to a susceptible neighbour. Fig. 3.1 (a) shows a pictorial representation of the rule describing the $\mathrm{CP}$. It illustrates the general form of these rules: a left-hand side (LHS) subgraph is transformed into a right-hand side (RHS) subgraph at a given rate per LHS subgraph. Such small subgraphs with given node states are also called motifs. Here, an infection occurs at a rate $p$ on links connecting nodes in the infective state I to nodes in the susceptible state $S$, i.e. on the S-I-link motif.

Adaptive changes of the network can be expressed in rules that alter the network topology depending on the node states. For instance, in the example of disease propagation, susceptible individuals may choose to avoid contact with the infected ones. This can be modelled by a rule that breaks links between infected and susceptible nodes at a certain rate (Fig. 3.1 (b)), or a rewiring rule, by which susceptible nodes seek to reconnect a link from an infectious neighbour to a random node in the network (Fig. 3.1 (d)). Taken together, such rules and the $\mathrm{CP}$ specify the dynamics of an adaptive network: the $\mathrm{CP}$ describes the topology-dependent dynamics of the node states, and the link deletion or rewiring rule provides a feedback of this dynamics on the network topology.

Note that the transformation rules describe stochastic dynamics, similar to chemical reactions. If the $\mathrm{CP}$ is the only rule in the model, the rate $p$ can be interpreted straightforwardly as the probability per unit time for a single S-I-link to be transformed into an I-I-link (possibly rescaling time to ensure $0 \leq p \leq 1$ ).

It should be emphasized that the symbolic notation of the transformation rules does not imply that the LHS subgraph has to be isolated in the network. The rules apply to all subgraphs of the form on the LHS regardless of how these are attached to the rest of the 


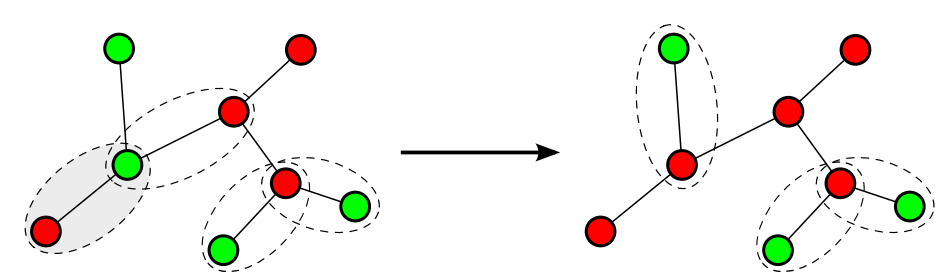

Figure 3.2.: Application of the $\mathrm{CP}$ rule to a network. The $\mathrm{LHS}$ subgraph of the $\mathrm{CP}$ rule matches all the circled links. Here, it is applied to the shaded link.

network (Fig. 3.2). This means that the $\mathrm{CP}$ rule with a constant rate as in Fig. 3.1 (a) is linear in the number of S-I-links, i.e. that an S-node with exactly two I-neighbours is twice as likely to switch to state I as one with a single I-neighbour.

\subsubsection{Update schemes}

A set of transformation rules does not define a unique model. It must be complemented by some instructions on how to apply the transformation rules to the network. To be more precise, an update scheme must be chosen. It specifies in which way the LHS subgraph should be selected from the network in order to apply a given rule. A full model specification must include both the transformation rules and the update scheme.

In general, LHS subgraphs containing more than a single node can be picked from the network in more than one way. As a specific example consider a network whose nodes represent interacting agents which hold one of two possible "opinions", so that the node state set is $\mathcal{S}_{2}=\{\mathrm{A}, \mathrm{B}\}$. The agents can change their opinion by adopting the opinion of the agents they interact with. This simple model is called the voter model, as it gives a stylized description of political opinion formation (Holley and Liggett 1975: Sood and Redner 2005).

The agents' opinion changes are described by the $\mathrm{CP}$ rule on the network, by which the nodes copy the states of their neighbours. To implement this rule, a link connecting two nodes in different states, i.e. an A-B-link, must be selected from the network, along which one of the nodes then copies the state of its neighbour. Such a link can be selected from the network in three different ways. First, one can pick a random node from the network and, if it has any neighbours in a different state, select at random one of them. If all its neighbours are in the same state, the procedure is repeated with another random node. This is called the direct node update scheme. Alternatively, one can reverse the order of selection: a random node is picked from the network, and then one of its neighbours in a different state (if any) copies its state. This is the reverse node update scheme. As a third possibility, one picks directly an A-B-link from all the A-B-links in the network, and then one of the nodes copies from the other. This is the link update scheme.

For transformation rules involving more complicated LHS subgraphs, there are in general even more different possible update schemes. These include different node update 


\begin{tabular}{l|ll} 
& $\left\langle k_{\text {target }}\right\rangle$ & $\left\langle k_{\text {source }}\right\rangle$ \\
\hline direct node update & $\langle k\rangle$ & $\left\langle k^{2}\right\rangle /\langle k\rangle$ \\
reverse node update & $\left\langle k^{2}\right\rangle /\langle k\rangle$ & $\langle k\rangle$ \\
link update & $\left\langle k^{2}\right\rangle /\langle k\rangle$ & $\left\langle k^{2}\right\rangle /\langle k\rangle$
\end{tabular}

Table 3.1.: Expected degrees of source and target nodes under the different update schemes for the $\mathrm{CP}$, in which the target node copies its state from the source node. In the direct node update scheme, the target node is selected at random and copies from a random neighbour (source). In the reverse node update scheme, the source is selected at random first, and a random target neighbour then copies its state. In the link update scheme, a link is selected at random, and either node copies from the other with equal probability.

schemes, which "build" the required LHS subgraph by successively picking random nodes and their neighbours, and the motif update scheme, which consists of selecting the LHS subgraph uniformly at random from all the matching LHS subgraphs in the network. The motif update scheme thus generalizes the link update scheme for higher LHS motifs.

The three possible update schemes for selecting an A-B-link differ in the way they select the node whose state is copied and the node which copies this state. In particular, the probability that a node of degree $k$ is selected depends on the update scheme. Whenever a node is selected by following a random link, either by following a link to a random neighbour in the node update schemes or directly in the link update scheme, the probability that it has exactly $k$ neighbours is proportional to $k p_{k}$, where $p_{k}$ is the degree distribution. This is because there are $k$ possibilities to select a node of degree $k$ in this way, as it can be reached via any of its $k$ links. By contrast, if a node is directly selected at random from all the nodes in the network, it has exactly $k$ neighbours with probability $p_{k}$.

The expected degree of the node whose state is being copied (the "source" node) and the node who copies (the "target" node) depends on the particular way they have been selected (Tab. 3.1). For a node picked at random from the whole population, it is simply $\langle k\rangle$, the average degree of the network. For a node selected by following a random link, however, it is $\left\langle k^{2}\right\rangle /\langle k\rangle$, where $\left\langle k^{2}\right\rangle$ is the second moment of the degree distribution (cf. section 2.1. Therefore, the expected degree of the source node is $\left\langle k^{2}\right\rangle /\langle k\rangle$ in the direct node update scheme and the link update scheme, while it is $\langle k\rangle$ in the reverse node update scheme. The expected degree of the target node, on the other hand, is $\left\langle k^{2}\right\rangle /\langle k\rangle$ in the reverse node update scheme and the link update scheme, and it is $\langle k\rangle$ in the direct node update scheme.

These differences in the expected degree of a node in the different update schemes result in potentially strong degree biases, which one has to keep in mind when modelling. As can be seen in Fig. 3.3, selecting a node by following a random link introduces a bias in favour of nodes with many neighbours in degree-heterogeneous networks. ${ }^{1}$ Therefore, using

\footnotetext{
${ }^{1}$ This also provides an explanation for the everyday experience that ' $y$ our friends have more friends than you do' (Feld 1991).
} 


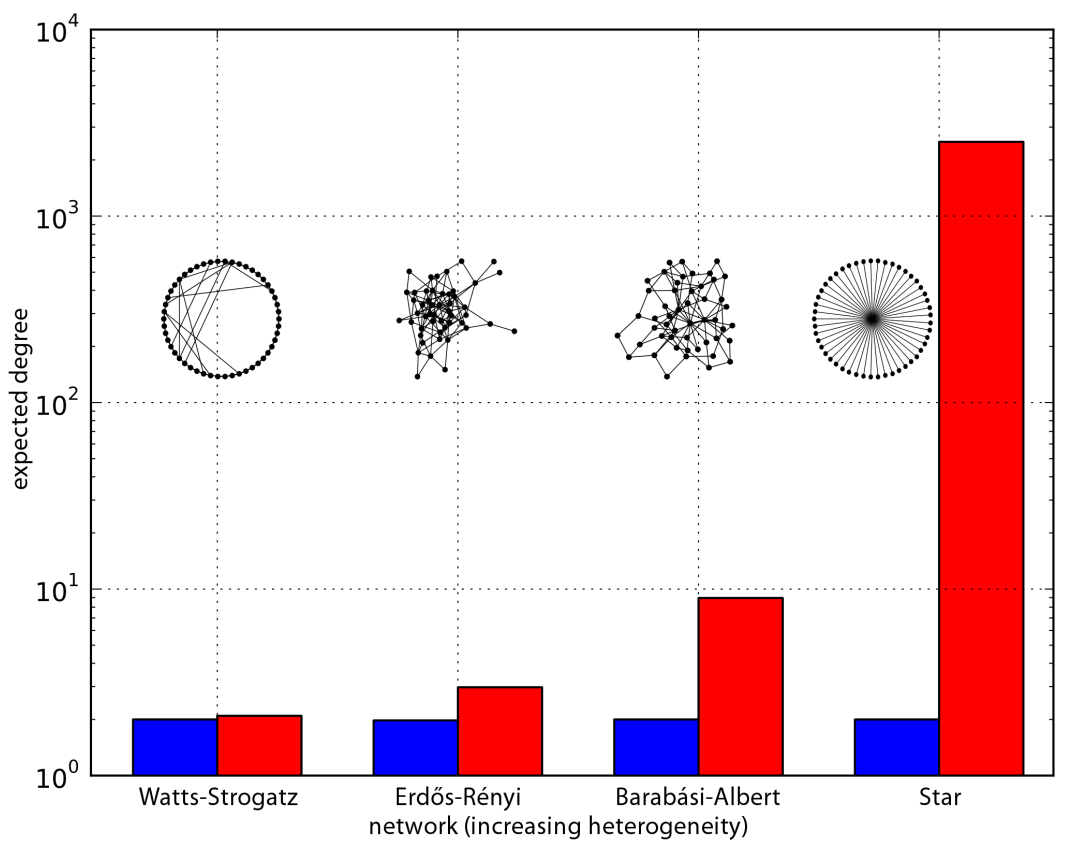

Figure 3.3.: Expected degrees of a randomly selected node (blue) and a random neighbour of a randomly selected node (red) for different network types with $N=5000$ nodes and $\langle k\rangle \approx 2$, whose overall appearance is illustrated by the network pictures. In strongly heterogeneous networks (the star network being the extreme case), the expected degree of a random neighbour can exceed that of a random node by several orders of magnitude. For the ws network, $p=0.1$ was used.

either of the three update schemes can lead to drastically different results (Castellano, 2005. Sood et al. 2008). This effect is especially pronounced in adaptive networks, where degree heterogeneity can arise naturally from the topological dynamics (Nardini et al. 2008).

The choice of an update scheme depends on the application. Depending on the particular system to be modelled, the node or link update scheme may be more appropriate. In the context of disease propagation, for instance, it is typically the case that there is a certain transmission probability per contact between susceptible and infectious agents. Because infection is thus an inherently link-related process and not "initiated" by either agent, using the link update scheme is the natural choice here.

In the adaptive-network models discussed in the present work, I use the link or motif update scheme except where noted otherwise.

\subsection{Adaptive-network simulation}

The dynamics of adaptive-network models can be conveniently studied by means of computer simulations. Due to the similarity between the microscopic transformation 


\section{Simulation and analysis of adaptive networks}

rules and chemical reactions, the basic algorithms used in stochastic simulations of chemical reactions can be adopted for the simulation of adaptive-network models.

Similar to chemical reactions, the transformation rules describe largely independent stochastic processes which produce a series of microscopic update events. ${ }^{2}$ The rates at which these events occur are determined by the transition rates associated with the transformation rules. Given an appropriate computer representation of the full network structure, the model dynamics can thus be simulated by directly applying the transformation rules in an asynchronous fashion according to their associated rates.

For the models considered in the present work, Gillespie's algorithm is employed to apply the transformation rules (Gillespie, 1976). Originally invented for the simulation of chemical reaction networks at low concentrations, this Monte Carlo algorithm has been successfully used in a wide range of different applications, including the modelling of biological populations (Renshaw 1991). In contrast to step-based simulation algorithms that go from one time step to the next time step, Gillespie's algorithm jumps directly from event to event, skipping over time steps in which no event takes place. Given the current state of the network and the transition rates associated to the transformation rules, it is straightforward to determine the time at which the next event will take place and what kind of event this will be, i.e. which transformation rule is to be applied (Press et al. 2007). The basic algorithm is thus the following:

1. Given the current state of the network, determine the transformation rule $\mathcal{R}$ to be applied next according to the transition rates and its associated time increment $\tau$.

2. Select at random an appropriate LHS subgraph for $\mathcal{R}$ according to the update scheme.

3. Apply $\mathcal{R}$ to the selected LHS subgraph, update the network configuration, and increase the time $t$ accordingly, setting $t \leftarrow t+\tau$.

4. If $t<t_{\max }$, repeat from step 1

The transformation rules are thus applied asynchronously to matching LHS subgraphs in the network, which is a natural realization of the real-world processes they describe. Nevertheless, one could also try to implement an alternative simulation scheme, in which the transformation rules are applied synchronously to all matching LHS. Such synchronous updating is routinely used for cellular automaton models on regular lattices (Ilachinski 2001). For adaptive networks, however, it is problematic for a number of reasons.

\footnotetext{
${ }^{2}$ Note that, while the molecules taking part in chemical reactions are indeed independent, this is only approximately true for the LHS subgraphs in the transformation rules, as they might overlap or be direct neighbours of each other. In the following, we consider large networks under asynchronous sequential updating, so that assuming independent transformation rules is well justified.
} 
First, it is often hard to imagine that the large number of units in a complex system are driven by an external "pacemaker" that dictates their local actions to occur synchronously ${ }^{3}$ Synchronous updating of the whole population may be appropriate in modelling the evolution of a biological species, where a sequence of well-defined generations can be observed in time. In many cases, however, asynchronous updating is a more natural choice.

Second, implementing synchronous changes in an adaptive network can lead to practical problems. When rewiring links, for instance, ambiguities may arise if a link $(i, j)$ is to be rewired to $(i, k)$ and $(l, j)$ simultaneously, which must be explicitly checked for and treated in a well-defined way $\left.\right|^{4}$ Moreover, synchronous updating can lead to spurious, artificial results, which depend strongly on the synchrony of the local update events and are immediately lost if the synchrony is not perfect (Nowak and May, 1992, Huberman and Glance, 1993: Cornforth et al. 2002, Newth and Cornforth, 2009).

Asynchronous updating is thus a natural and practical choice for many applications and in particular for the models considered in the present work. In the following, I use asynchronous updating except where noted otherwise.

The rule-based simulation approach used here is closely related to the concept of generative network automata in computer science (Sayama and Laramee 2009). In the framework of generative network automata, a labelled graph is evolved by sequential "graph rewriting" events, each of which is defined by an extraction rule selecting a specific subgraph that is to be altered, and a replacement rule that produces a new subgraph from the selected one and determines how to embed this new subgraph in the network. Generative network automata form a more general framework for the computer simulation of the models considered in the present work, which are specified in terms of the transformation rules and the update scheme. In this sense, the update scheme corresponds to the extraction rules, as it defines how the LHS subgraphs of the transformation rules are selected from the network, and the transformation rules themselves correspond to the replacement rules. A similar graph rewriting formalism was previously introduced as an extension of cellular automata, which can be applied synchronously (Tomita et al. 2002 , 2009) or asynchronously (Tomita et al., 2007).

In order to implement the transformation rules according to Gillespie's algorithm, appropriate data structures are needed that provide a computer representation of the full network configuration, including the network topology and the node states at each step. For an efficient simulation, they must allow to perform the typical update processes at relatively low computational cost. The most commonly used processes in adaptive networks are the addition or removal of nodes and links and changes in the nodes' states depending on their neighbourhood. Thus, addition and removal of nodes and links and inspecting a node's neighbourhood are required to be fast operations. In order to directly

\footnotetext{
${ }^{3}$ Synchronized behaviour may of course be achieved dynamically through the interactions among the individual units, as for instance in networks of coupled oscillators. This emergent phenomenon, however, is quite different from explicit synchronous updating.

${ }^{4}$ For an example of synchronous link dynamics that avoids such ambiguities, see (Tomita et al. 2002).
} 


\section{Simulation and analysis of adaptive networks}

implement the transformation rules specified by the given model, enumerating typical LHS subgraphs must also be fast, such as enumerating all nodes in a given state or all links connecting two nodes in given states.

The data structures used to represent graphs in computer science are typically tailored towards the efficient implementation of certain algorithms, as for instance graph traversal, search, or finding the shortest paths (Sedgewick, 2002. Siek et al., 2002). In many cases, these algorithms work on graphs with a fixed number of nodes. Moreover, node and link states are not considered specifically in many algorithms, so that efficient access to these properties is usually not of major concern. For the efficient simulation of adaptive networks, existing data structures allowing for the fast traversal and manipulation of a node's neighbourhood must therefore be extended to support efficient access to the node and link states and also state-specific access to the nodes and links.

The simulations of the adaptive-network models considered in the present work have been implemented in $\mathrm{C}++$. We store all the nodes, links, and required larger motifs in a custom-made, index-based container similar to std: : vector, which can hold items in different discrete categories (states) $4_{5}^{5}$ It ensures that items in the same category are stored in contingent memory, and provides both index-based and category-based access, so that selecting a random item in a given category can be achieved in constant time, for instance. The network structure is modelled directly in memory using nodes and links as the basic entities in a double adjacency set representation, in which each node keeps a std: : set of pointers to its incoming and outgoing links. At the cost of some memory overhead, addition and removal of links is thus achieved in logarithmic time. Depending on the complexity of the transformation rules, simulating large adaptive networks of $N=10^{6}$ and more is feasible in our implementation.

\subsection{Emergent-level description}

In order to gain further insight in the behaviour of an adaptive-network model, investigation beyond computer simulation of the transformation rules is desirable. The large number of degrees of freedom in an adaptive network and the stochastic nature of the transformation rules make it prohibitively difficult to follow the system's microscopic dynamics. However, the microscopic properties of the system are usually of minor interest. Instead, one is often interested in the behaviour of macroscopic quantities, such as the total number of infected nodes, for instance, in a model of disease propagation.

Thus, suitable projections of the high-dimensional phase space spanned by the possible network configurations (including both the topological configuration and the nodes' states) are needed. In other words, one seeks an appropriate macroscopic model that captures the emergent behaviour arising from the microscopic transformation rules.

For the models considered in the present work, the observables of most interest are

\footnotetext{
${ }^{5}$ The source code of the largenet2 library developed in the course of this thesis can be found at http://www.github.com/rincedd/largenet2
} 
the so-called network moments. These are the densities of small subgraphs (motifs), such as the nodes in a given state or the links connecting nodes in given states. The number of links involved in the network moment defines its order: node densities are moments of zeroth order, link densities are moments of first order, etc. In the following I discuss how these moments can be used in a low-dimensional, emergent-level description of the network dynamics in terms of deterministic ordinary differential equations (ODE $)$. For the sake of clarity, I will use a simple example model of disease propagation to illustrate the method.

\subsubsection{From rules to moment equations}

The possible changes in any configuration of an adaptive network are defined by the transformation rules, which determine the events that can take place in the network, and the update scheme, which determines in which way these events take place. For instance, the $\mathrm{CP}$ rule under the link update scheme states that $\mathrm{S}$-nodes can be transformed into I-nodes via the S-I-links in the network at a constant rate $p$ per link. Due to the stochastic nature of the rule, this means that, at any time $t$, such state change events occur on average at a rate $p$ times the average number of S-I-links at that time, where the average is taken over many realizations. Using this interpretation, one can write a rate equation for the expectation value of a macroscopic observable $f$, starting from the general form

$$
\frac{\mathrm{d}}{\mathrm{dt}}\langle f(t)\rangle=\sum_{\text {events } \epsilon} \text { rate of } \epsilon \times \text { change in } f \text { due to } \epsilon,
$$

where the sum runs over all possible events described by the transformation rules (Rand, 1999). Here, both the average rate of an event and its associated change in $f$ are instantaneous, that is to say, they generally depend on the (average) network configuration at time $t$. Equation (3.1) describes the time evolution of the phase space average of an observable $f$. Therefore, it can be derived in principle from the master equation of the system (Barrat et al. 2008). Nevertheless, it is often more convenient to directly construct balance equations for the macroscopic observables, especially if one is interested in network moments. In this case, such balance equations can be derived intuitively from the transformation rules.

To be specific, consider the $\mathrm{CP}$ rule in Fig. 3.1 (a) together with the recovery rule

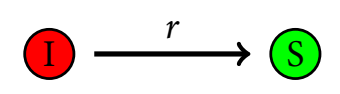

under the motif update scheme for a (non-adaptive) network model of susceptible-infected-susceptible (SIS) disease propagation in a population of $N$ nodes. The nodes can be susceptible to the disease or infectious, denoted by $\mathcal{S}=\{\mathrm{S}, \mathrm{I}\}$. Infection occurs through the contact of individuals at a constant rate $p$ per S-I-link, i.e. by the CP rule. Infected nodes recover and become susceptible again at a rate $r$, which does not depend on their neighbourhood. 


\section{Simulation and analysis of adaptive networks}

We are interested in a low-dimensional, deterministic description of the average system trajectories of this SIS model in terms of a system of ODE; for the network moments. Obviously, the fraction of infectious nodes, denoted by [I], is of particular interest. We write $[\sigma]$ for the fraction of nodes in state $\sigma$ and $\left[\sigma \sigma^{\prime}\right]$ for the number of links connecting nodes in states $\sigma$ and $\sigma^{\prime}$ divided by $N$. Thus, the network moments are subject to the normalization conditions $[\mathrm{S}]+[\mathrm{I}]=1$ and $[\mathrm{SS}]+[\mathrm{SI}]+[\mathrm{II}]=\langle k\rangle / 2$, stating that the total numbers of nodes and links are conserved by the transformation rules.

According to 3.1 , the change in the average fraction [I] of infectious nodes in the network is given by

$$
\frac{\mathrm{d}}{\mathrm{dt}}[\mathrm{I}]=\sum_{\text {infection }} p \times N^{-1}-\sum_{\text {recovery }} r \times N^{-1}
$$

where the first sum runs over all infection events, which increase [I] by $N^{-1}$ each, and the second sum runs over all recovery events, which decrease [I] by $N^{-1}$. At any time $t$, infection occurs at an instantaneous rate $p$ on any S-I-link, and recovery occurs at a rate $r$ on any infected node. Therefore, the sums can be replaced by sums over S-I-links and I-nodes, respectively, yielding ${ }^{6}$

$$
\frac{\mathrm{d}}{\mathrm{dt}}[\mathrm{I}]=\sum_{\sigma_{i j}=\mathrm{SI}} p N^{-1}-\sum_{\sigma_{i}=\mathrm{I}} r N^{-1}=p[\mathrm{SI}]-r[\mathrm{I}] .
$$

This equation is a direct translation of the transformation rules: infectious nodes are lost by recovery, whereas one infectious node is gained on average per infection event on S-I-links. Note that this equation is valid only in the thermodynamic limit of infinite system size, because we treat [I] and [SI] as continuous density variables and neglect the effect of stochastic fluctuations.

Equation (3.2) is not yet a closed ODE and needs to be complemented by an additional dynamical equation for [SI]. Taking into account the possible infection and recovery events, the rate equation for $[\mathrm{SI}]$ is

$$
\frac{\mathrm{d}}{\mathrm{dt}}[\mathrm{SI}]=\frac{r}{N} \sum_{\sigma_{i}=\mathrm{I}}\left\{n_{i}(\mathrm{I})-n_{i}(\mathrm{~S})\right\}+\frac{p}{N} \sum_{\sigma_{i j}=\mathrm{SI}}\left\{n_{i}(\mathrm{~S})-n_{i}(\mathrm{I})\right\}
$$

where $n_{i}(\sigma)$ denotes the number of node i's neighbours in state $\sigma$. The first term balances the gain and loss in S-I-links due to recovery of I-nodes. One S-I-link is gained for every I-node connected to a recovering I-node, and lost for every S-node connected to a recovering I-node, which contributes $+N^{-1}$ and $-N^{-1}$ to [SI], respectively. The second term accounts for the direct and indirect gains and losses due to infection on S-I-links, which creates new S-I-links from every additional link to an S-neighbour of the S-node that is to be infected, and transforms every S-I-link to an I-neighbour of this node into an I-I-link (Fig. 3.4).

\footnotetext{
${ }^{6}$ For simplicity, we write the summation over all nodes $i$ in state $\sigma$ as $\sum_{\sigma_{i}=\sigma}$ and the summation over all
} 


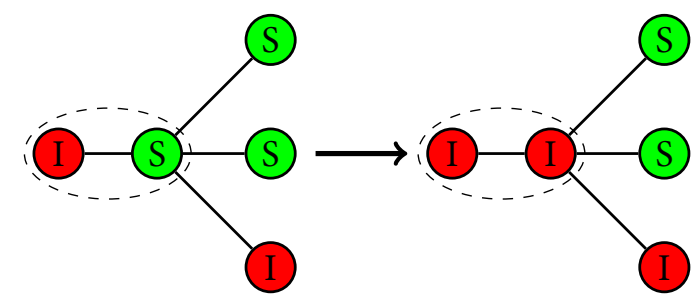

Figure 3.4.: Infection on an S-I-link affects the state of all additional links connected to the S-node. Here, two S-S-links are destroyed and two I-I-links are created by the infection, while there is no net change in the number of S-I-links.

As they only involve the constant rates $p$ and $r$, the sums in (3.3) can be carried out straightforwardly, yielding

$$
\begin{aligned}
\frac{\mathrm{d}}{\mathrm{dt}}[\mathrm{SI}] & =2 r[\mathrm{II}]-r[\mathrm{SI}]+\underbrace{\frac{p}{N} \sum_{\sigma_{i j}=\mathrm{SI}} n_{i}(\mathrm{~S})}_{\text {indirect gain }}-\underbrace{\frac{p}{N} \sum_{\sigma_{i j}=\mathrm{SI}}\left(n_{i}(\mathrm{I})-1\right)}_{\text {indirect loss }}-\underbrace{\frac{p}{N} \sum_{\sigma_{i j}=\mathrm{SI}} 1}_{\text {direct loss }} \\
& =2 r[\mathrm{II}]-r[\mathrm{SI}]+p[\mathrm{SSI}]-2 p[\mathrm{ISI}]-p[\mathrm{SI}],
\end{aligned}
$$

where $\left[\sigma \sigma^{\prime} \sigma^{\prime \prime}\right]$ denotes the number of $\sigma-\sigma^{\prime}-\sigma^{\prime \prime}$-triplets, i.e. the number of second-order motifs consisting of three nodes in a row, divided by $N$. Deriving in the same way a similar equation for [SS], we obtain

$$
\begin{aligned}
\frac{\mathrm{d}}{\mathrm{dt}}[\mathrm{I}] & =p[\mathrm{SI}]-r[\mathrm{I}], \\
\frac{\mathrm{d}}{\mathrm{dt}}[\mathrm{SI}] & =2 r[\mathrm{II}]-r[\mathrm{SI}]+p[\mathrm{SSI}]-2 p[\mathrm{ISI}]-p[\mathrm{SI}], \\
\frac{\mathrm{d}}{\mathrm{dt}}[\mathrm{SS}] & =r[\mathrm{SI}]-p[\mathrm{SSI}] .
\end{aligned}
$$

At this point, an important property of the $\mathrm{CP}$ becomes obvious. In $3.5-3.7$, several terms associated to infection involve moments of higher order than the order of the equation. That is, the infection-related terms in the equation for the zeroth-order moment [I] involve the first-order moment [SI]. The equations for the link motifs [SI] and [SS] involve the second-order moments [SSI] and [ISI], and so on. This is a general property of the $\mathrm{CP}$ in this type of moment expansion equations: an equation describing the change in a moment of order $n$ depends on moments of order $n+1$. Writing equations for these higher-order moments therefore results in an infinite cascade of ODE. In order to close the ODE system, a truncation at some order is necessary, which can be achieved using suitable approximations (e.g. Do and Gross 2009). I will discuss such moment closure approximations in section 3.3 .2

links $(i, j)$ connecting nodes in state $\sigma$ and $\sigma^{\prime}$ as $\sum_{\sigma_{i j}=\sigma \sigma^{\prime}}$. More precisely, we have for any $x_{i}$ and $y_{i j}$ that $\sum_{\sigma_{i}=\sigma} x_{i} \equiv \sum_{i} \delta_{\sigma_{i} \sigma} x_{i}$ and $\sum_{\sigma_{i j}=\sigma \sigma^{\prime}} y_{i j} \equiv \sum_{(i, j)} \delta_{\sigma_{i} \sigma} \delta_{\sigma_{j} \sigma^{\prime}} y_{i j}$. 


\section{Simulation and analysis of adaptive networks}

\section{Non-linear rates}

For the simple processes considered so far, the corresponding rate equations could be derived rather intuitively. The reason for that is that the rates associated with the transformation rules are simple constants and do not depend on the specific realization of the LHS subgraph in the network. In particular, they depend linearly on the local topology defined by the LHS

The $\mathrm{CP}$ is a special case of the more general rule

$$
\text { (S) } \stackrel{g\left(\mathcal{N}_{i}\right)}{\longrightarrow} \mathrm{I}_{i}
$$

describing the state change of a node $i$ at an instantaneous rate $g\left(\mathcal{N}_{i}\right)$, which is some function of the node's topological neighbourhood $\mathcal{N}_{i}$. In general, this rate will vary among the nodes because they differ in their local neighbourhoods. The change in, e.g., $[\mathrm{S}]$ is then given by

$$
\frac{\mathrm{d}}{\mathrm{dt}}[\mathrm{S}]=-\frac{1}{N} \sum_{\sigma_{i}=\mathrm{S}} g\left(\mathcal{N}_{i}\right) .
$$

Depending on the specific form of $g\left(\mathcal{N}_{i}\right)$, the summation may be simplified to involve only macroscopic observables or not. For instance, consider the rate

$$
g_{1}\left(\mathcal{N}_{i}\right)=p n_{i}(\mathrm{I})
$$

which is linear in the number $n_{i}(\mathrm{I})$ of infectious neighbours of the susceptible node $i$. Therefore,

$$
\frac{\mathrm{d}}{\mathrm{dt}}[\mathrm{S}]=-\frac{1}{N} \sum_{\sigma_{i}=\mathrm{S}} g_{1}\left(\mathcal{N}_{i}\right)=-\frac{p}{N} \sum_{\sigma_{i}=\mathrm{S}} n_{i}(\mathrm{I})=-p[\mathrm{SI}] .
$$

Comparing this expression with the change in $[\mathrm{S}]$ due to the $\mathrm{CP}$ as in Fig. 3.1 (a), it is obvious that both the general rule with rate $g_{1}\left(\mathcal{N}_{i}\right)$ and the $\mathrm{CP}$ lead to the same macroscopic description. One can thus expect that the general rule gives rise to the same macroscopic behaviour as the $\mathrm{CP}$ rule under the direct node update scheme, although their microscopic implementation is different. The general rule may therefore be written equivalently as the $\mathrm{CP}$ rule with an S-I-link as the LHS motif.

In general, however, the summation in $(3.8)$ cannot be carried out if the transition rates are non-linear functions of $\mathcal{N}_{i}$. Nevertheless, they can sometimes be expanded in a power series in the number of, say, I-neighbours of the focal node. In this case the corresponding rules may be replaced by a set of surrogate rules that involve linear transition rates but include higher-order LHS motifs. On the level of the ODE description, these surrogate rules are expected to show the same macroscopic behaviour as the original non-linear rules, as discussed in appendix $\mathrm{A}$ 


\subsubsection{Moment closure approximations}

Transformation rules of the CP-type lead to an infinite cascade of dynamical equations because the change in a network moment of order $n$ depends on moments of order $n+1$. Therefore, suitable approximations are necessary to close the ODE system. Using such approximations, the moments of highest order are expressed in terms of lower-order moments.

The simplest approximation of this kind is the mean-field approximation, which closes the ODE system at zeroth order. It assumes that the system is homogeneous, so that the local density of I-neighbours of susceptible nodes equals the global density [I]. Thus, $[\mathrm{SI}] \approx\langle k\rangle[\mathrm{S}][\mathrm{I}]$ and 3.2$)$ can be closed as

$$
\frac{\mathrm{d}}{\mathrm{dt}}[\mathrm{I}]=p\langle k\rangle[\mathrm{S}][\mathrm{I}]-r[\mathrm{I}]=(p\langle k\rangle-r)[\mathrm{I}]-p\langle k\rangle[\mathrm{I}]^{2},
$$

where we have used the normalization condition. In the mean-field approximation, all structural properties of the network are neglected except the mean degree $\langle k\rangle$. This means that transformation rules creating or destroying links or larger motifs, such as in Fig. 3.1 (b)-(d), cannot be taken into account within this approximation.

To be able to capture some features of the topological dynamics, the moment equations must be closed at a higher order than in the mean-field approximation. The simplest extension beyond the mean-field approximation is keeping the dynamical equations for the link motifs and closing at first order. This is done using the pair approximation (PA) (Keeling et al. 1997, Rand, 1999. Gross et al., 2006), using which the triplet moments $\left[\sigma \sigma^{\prime} \sigma^{\prime \prime}\right]$ are expressed in terms of links and nodes.

To derive the $\mathrm{PA}$ the triplet moments are written as

$$
\mu_{\sigma \sigma^{\prime \prime}}\left[\sigma \sigma^{\prime} \sigma^{\prime \prime}\right]=\mu_{\sigma \sigma^{\prime}}\left[\sigma \sigma^{\prime}\right] Q\left(\sigma^{\prime \prime} \mid \sigma^{\prime} \sigma\right)
$$

where $\mu_{\sigma \sigma^{\prime}}=1+\delta_{\sigma \sigma^{\prime}}$ accounts for the double-counting of symmetric triplets. Here, $Q\left(\sigma^{\prime \prime} \mid \sigma^{\prime} \sigma\right)$ denotes the average number of additional $\sigma^{\prime \prime}$-nodes connected to the $\sigma^{\prime}$ node in a $\sigma-\sigma^{\prime}$-link. Then two assumptions are made. First, one assumes that, given a $\sigma-\sigma^{\prime}$-link, the number of additional $\sigma^{\prime \prime}$-neighbours of the $\sigma^{\prime}$-node does not depend on the fact that it has already at least one $\sigma$-neighbour. In other words, one assumes that there is no correlation between next-nearest neighbours in the network. Second, one assumes that the $\sigma^{\prime}-\sigma^{\prime \prime}$-links are distributed homogeneously in the network. This is similar to the meanfield assumption for the zeroth-order approximation, where one assumes that the nodes in a given state are distributed homogeneously. Combining these two assumptions, the average number of additional $\sigma^{\prime \prime}$-neighbours of the $\sigma^{\prime}$-node, $Q\left(\sigma^{\prime \prime} \mid \sigma^{\prime} \sigma\right)$, is approximated as the expected number of $\sigma^{\prime \prime}$ - $\sigma^{\prime}$-links drawn from a binomial distribution with the singleevent probability $P\left(\sigma^{\prime \prime} \mid \sigma^{\prime}\right)$ of finding a $\sigma^{\prime \prime}$-node connected to a $\sigma^{\prime}$-node in the network,

$$
Q\left(\sigma^{\prime \prime} \mid \sigma^{\prime} \sigma\right) \approx\left\langle q_{\sigma^{\prime}}\right\rangle P\left(\sigma^{\prime \prime} \mid \sigma^{\prime}\right)
$$




\section{Simulation and analysis of adaptive networks}

Here, $\left\langle q_{\sigma^{\prime}}\right\rangle$ is the mean excess degree of the $\sigma^{\prime}$-nodes, i.e. the number of additional links connected to a $\sigma^{\prime}$-node selected by following a random link, 7 each of which is a $\sigma^{\prime}-\sigma^{\prime \prime}$ link with probability $P\left(\sigma^{\prime \prime} \mid \sigma^{\prime}\right)$. As the $\sigma^{\prime}-\sigma^{\prime \prime}$-links are assumed to be homogeneously distributed, this probability is given by

$$
P\left(\sigma^{\prime \prime} \mid \sigma^{\prime}\right)=\frac{\mu_{\sigma^{\prime} \sigma^{\prime \prime}}\left[\sigma^{\prime} \sigma^{\prime \prime}\right]}{\sum_{\sigma} \mu_{\sigma^{\prime} \sigma}\left[\sigma^{\prime} \sigma\right]}=\frac{\mu_{\sigma^{\prime} \sigma^{\prime \prime}}\left[\sigma^{\prime} \sigma^{\prime \prime}\right]}{\left\langle k_{\sigma^{\prime}}\right\rangle\left[\sigma^{\prime}\right]} .
$$

Thus in the PA the triplet moments are approximated as

$$
\left[\sigma \sigma^{\prime} \sigma^{\prime \prime}\right] \approx \frac{\left\langle q_{\sigma^{\prime}}\right\rangle}{\left\langle k_{\sigma^{\prime}}\right\rangle} \frac{\left[\sigma \sigma^{\prime}\right]\left[\sigma^{\prime} \sigma^{\prime \prime}\right]}{\left[\sigma^{\prime}\right]} \frac{\mu_{\sigma \sigma^{\prime}} \mu_{\sigma^{\prime} \sigma^{\prime \prime}}}{\mu_{\sigma \sigma^{\prime \prime}}},
$$

where $\left\langle k_{\sigma^{\prime}}\right\rangle$ and $\left\langle q_{\sigma^{\prime}}\right\rangle$ denote the mean degree and the mean excess degree of the $\sigma^{\prime}$ nodes. For simplicity one often assumes $\left\langle q_{\sigma^{\prime}}\right\rangle \approx\langle q\rangle$ and $\left\langle k_{\sigma^{\prime}}\right\rangle \approx\langle k\rangle$ instead of using the state-specific quantities, as the mean excess degree $\langle q\rangle$ can be computed from the the state-independent degree distribution $p_{k}$ as $\langle q\rangle=\left\langle k^{2}\right\rangle /\langle k\rangle-1$. The validity of this simplification should, however, be tested for each particular application, as the coupling of topological and node state dynamics can give rise to strong correlations between a node's state and degree in adaptive networks.

Within the PA $3.5-3.7$ become

$$
\begin{aligned}
\frac{\mathrm{d}}{\mathrm{dt}}[\mathrm{I}] & =p[\mathrm{SI}]-r[\mathrm{I}], \\
\frac{\mathrm{d}}{\mathrm{dt}}[\mathrm{SI}] & =2 r[\mathrm{II}]+2 p \kappa \frac{[\mathrm{SS}][\mathrm{SI}]}{[\mathrm{S}]}-r[\mathrm{SI}]-p[\mathrm{SI}]-p \kappa \frac{[\mathrm{SI}]^{2}}{[\mathrm{~S}]}, \\
\frac{\mathrm{d}}{\mathrm{dt}}[\mathrm{SS}] & =r[\mathrm{SI}]-2 p \kappa \frac{[\mathrm{SS}][\mathrm{SI}]}{[\mathrm{S}]},
\end{aligned}
$$

with $\kappa=\langle q\rangle /\langle k\rangle$. This forms a closed ODE system together with the link conservation condition $[\mathrm{SS}]+[\mathrm{II}]+[\mathrm{SI}]=\langle k\rangle / 2$. It can now be (numerically) integrated to obtain the time evolution of the network moments.

The system's long-term behaviour can be characterized by applying the tools from the theory of dynamical systems to analyse the stationary states, their stability, the dependence on parameters, and the phase space structure of the ODE system (e.g., Kuznetsov, 1998, Strogatz, 2001. Guckenheimer and Holmes, 2002). For instance, consider the two stationary solutions of equations 3.14$)-3.16)$ which lie within the physical boundaries $:^{8}$ the trivial healthy state with zero infectious nodes, $[\mathrm{I}]^{*}=0$, and the endemic state characterized by a non-vanishing prevalence,

$$
[\mathrm{I}]^{*}=\frac{2 r(\kappa-1)-p \kappa(1+\langle k\rangle)+\kappa \sqrt{p^{2}(\langle k\rangle-1)^{2}+4 r p(1+\langle k\rangle(\kappa-1))}}{2 r(\kappa-1)^{2}-2 p \kappa} .
$$

\footnotetext{
${ }^{7}$ The state-specific mean excess degree $\left\langle q_{\sigma}\right\rangle$ is computed from the degree distribution of the $\sigma$-nodes, $p_{k}^{\sigma}$, as $\sum_{k} k(k+1) p_{k}^{\sigma} /\left\langle k_{\sigma}\right\rangle[\sigma]$, where $p_{k}^{\sigma}$ is the probability that a randomly selected node is in state $\sigma$ and has exactly $k$ neighbours (cf. section 2.1 .

${ }^{8}$ For simplicity, I do not show the expressions for $[\mathrm{SI}]^{*}$ and $[\mathrm{SS}]^{*}$.
} 


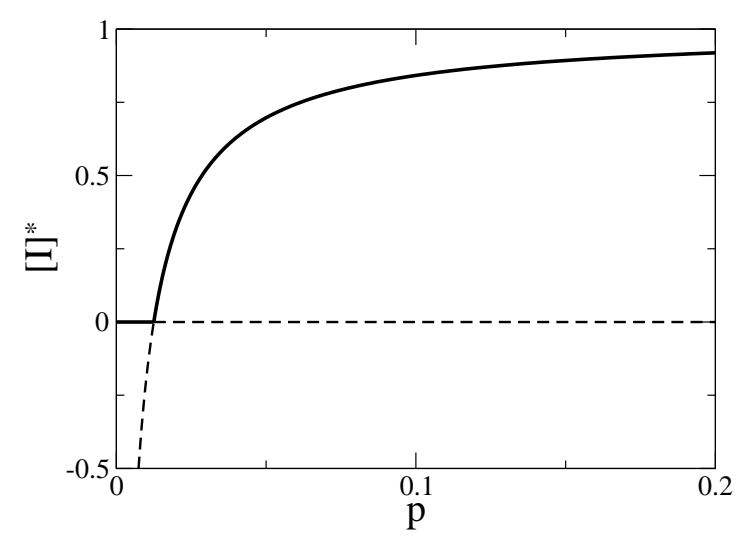

Figure 3.5.: Bifurcation diagram of the non-adaptive SIS model showing the stationary fraction of infectious nodes $[\mathrm{I}]^{*}$ as a function of the infection rate $p$. The healthy state $[\mathrm{I}]^{*}=0$ is stable below a critical infection rate, at which the endemic state $[\mathrm{I}]^{*}>0$ becomes stable in a transcritical bifurcation. The unstable stationary state $[\mathrm{I}]^{*}<0$ is a non-physical solution of the PA equations. Parameters: $r=0.05,\langle k\rangle=4, \kappa=1$.

At low infection rates $p$, the healthy state is stable, whereas the endemic state is stable at high infection rates. Indeed, linear stability analysis reveals a transcritical bifurcation ${ }^{9}$ at an infection rate $p_{c}=r /\langle k\rangle \kappa=r /\langle q\rangle$, in which the two stationary states exchange their stability (Fig. 3.5). This critical infection rate is called the epidemic threshold (Hethcote, 2000, Pastor-Satorras and Vespignani 2001a b).

\section{Limitations and improvements of the PA}

Using the moment closure equations, both the time evolution and the long-term dynamics of an adaptive-network model can be studied within the scope of the underlying assumptions. To assess the accuracy of the moment closure approach, the predictions of the approximate ODE description must be compared with experimental realizations of the model, i.e. with experimental data or computer simulations. In this way, the validity of the underlying assumptions can be tested, and improved approximations can be developed in cases where these assumptions are violated.

The PA relies on two essential assumptions: the independence of next-nearest neighbours and the sufficiently homogeneous distribution of the different link types across the network. The first assumption is often violated when the network is clustered and many closed triangles are present. Assuming that two next-nearest neighbours are independent is clearly not justified in this case because they are likely to be direct neighbours of each other. For clustered networks, the $\mathrm{PA}$ can be modified to include a heuristic accounting for the correlations induced by such clustering. Denoting by $\phi$ the average clustering

\footnotetext{
${ }^{9}$ For a brief overview of the basic concepts of dynamical systems theory used in this work, see appendix $B$
} 


\section{Simulation and analysis of adaptive networks}

coefficient of the network (cf. 2.1), the second-order moments are thus approximated as

$$
\left[\sigma \sigma^{\prime} \sigma^{\prime \prime}\right] \approx \frac{\langle q\rangle}{\langle k\rangle} \frac{\mu_{\sigma \sigma^{\prime}} \mu_{\sigma^{\prime} \sigma^{\prime \prime}}}{\mu_{\sigma \sigma^{\prime \prime}}}\left\{(1-\phi) \frac{\left[\sigma \sigma^{\prime}\right]\left[\sigma^{\prime} \sigma^{\prime \prime}\right]}{\left[\sigma^{\prime}\right]}+\phi \frac{\left[\sigma \sigma^{\prime}\right]\left[\sigma^{\prime} \sigma^{\prime \prime}\right]\left[\sigma^{\prime \prime} \sigma\right]}{[\sigma]\left[\sigma^{\prime}\right]\left[\sigma^{\prime \prime}\right]} \frac{\mu_{\sigma^{\prime \prime} \sigma}}{\langle k\rangle}\right\} .
$$

The second term in this expression is a heuristic to account for the closed triangles in the network, motivated by the so-called Kirkwood approximation for three-point correlation functions in chemical physics (Keeling et al., 1997, Morris, 1997, Rand, 1999, Singer. 2004). In the present work, however, I concentrate on networks with low clustering and transformation rules with no significant bias towards creating clustered topologies, so that the original $\mathrm{PA}$ expression 3.13 is expected to be sufficient.

The second assumption in the $\mathrm{PA}$ approach is homogeneity in the link distributions, so that the local probability $P_{i}\left(\sigma^{\prime} \mid \sigma\right)$ of finding a $\sigma^{\prime}$-node connected to a given node $i$ in state $\sigma_{i}=\sigma$ can be approximated as the global density of $\sigma$ - $\sigma^{\prime}$-links per $\sigma$-node, namely $P\left(\sigma^{\prime} \mid \sigma\right)=\mu_{\sigma \sigma^{\prime}}\left[\sigma \sigma^{\prime}\right] /\left\langle k_{\sigma}\right\rangle[\sigma]$ as in 3.12$)$. This assumption fails if the transformation rules tend to create strongly localized structures. For instance, this is the case in the early stages of disease propagation in a fixed network of susceptible individuals with a single initially infectious node. Clearly, the infection spreads first to the immediate neighbourhood of this node, leading to a localized density of infectious nodes. Then, the probability of finding an infectious node connected to a susceptible node within this initial cluster is much larger than the global link density because most of the S-nodes are connected to other S-nodes in the network. As the number of infectious nodes increases and the disease spreads across the network, this discrepancy often decreases, so that the assumption of homogeneity may become valid again. For this reason, the standard PA approach typically fails to predict the transient dynamics of disease propagation and similar processes, whereas it often gives good estimates for, e.g., the stationary prevalence of infectious nodes.

In order to account for the transient localized effects in spreading processes, the $\mathrm{PA}$ approach can be modified to explicitly describe the link densities within the initial cluster. This method predicts the transient dynamics much better than the PA, but it was found to give less accurate results for the system's long-term behaviour (Bauch. 2005). Improving the $\mathrm{PA}$ is thus also a matter of the particular application and the research questions to be addressed.

A natural way to further improve the accuracy of the moment equations seems to be closing them at second or higher order. Instead of closing at the level of links, for instance, one can also include equations for the triplets and approximate the occurring 4-node motifs in terms of triplets and links. However, although the moment expansion up to infinite order would, in principle, be exact, truncating at a higher order is not guaranteed to reduce the approximation error as compared to a truncation at lower order. Moreover, approximation schemes for moments of order three and greater are generally not unique. A particular choice may be justified by the transformation rules of the model in question (Bauch 2005, Kimura and Hayakawa, 2008. Peyrard et al. 2008, House et al. 2009. Rozhnova and Nunes 2009. Demirel et al. 2011b). 
In most of the present work, I focus on the long-term dynamics of different adaptivenetwork models in which only little clustering is expected. Therefore, I typically use the ordinary $\mathrm{PA}$ given by (3.13) in the analytical description of these models.

\subsubsection{Other approaches}

In addition to the procedure of moment expansion and closure, several other approaches for a macroscopic, approximate description of dynamical processes on networks have been proposed in the recent past. One can broadly identify three paths being pursued: the mesoscopic description in terms of less coarse-grained dynamical variables than the global moments (Pugliese and Castellano, 2009, Marceau et al., 2010, Baronchelli and Pastor-Satorras 2010. House and Keeling 2011), the use of an ensemble picture based on "annealed" adjacency matrices (Guerra and Gómez-Gardeñes. 2010), and the formulation of the model as a message passing problem (Karrer and Newman, 2010, Hébert-Dufresne et al. 2010).

These approaches have been successfully applied to describe spreading dynamics on non-adaptive networks, while their application to adaptive networks is still limited. So far, only the mesoscopic description and the ensemble approach have also been applied to adaptive networks. In both cases, higher accuracy compared to the moment closure procedure is obtained at the expense of losing the low dimensionality of, e.g., the $\mathrm{PA}$ approach.

In the mesoscopic description, for instance, less coarse-grained, degree-dependent dynamical variables are used instead of the global moments to obtain higher precision. In the SIS model on an adaptive network, the long-term dynamics can thus be predicted with high accuracy even for very sparse networks with low $\langle k\rangle$, for which the PA yields less accurate results. This has been shown by Marceau et al. (2010), who used as dynamical variables the quantities $S_{k l}$ and $I_{k l}$, respectively, which count the numbers of S- and I-nodes with exactly $k$ neighbours, of which $l \leq k$ are in the infectious state I. In this approach, a system of $O\left(k_{\max }^{2}\right) \mathrm{ODE}$ must be solved, where $k_{\max }$ is a cut-off at the maximum node degree that is to be taken into account. This approach is thus not easily scalable to models with three or more node states (leading to cubic or higher complexity), or cases where high node-degrees emerge dynamically.

Similar scalability problems arise in the ensemble approach, in which the central dynamical quantity is the so-called annealed adjacency matrix, which describes an ensemble of networks subject to some constraints (Guerra and Gómez-Gardeñes, 2010). It consists of the probabilities $\mathcal{A}_{i j}$ that nodes $i$ and $j$ are connected in a network drawn from this ensemble. The matrix $\mathcal{A}$ can thus be obtained as the ensemble average of all possible adjacency matrices or constructed directly from the network constraints. The probabilities $\mathcal{A}_{i j}$ can then be used in the dynamical equations for, e.g., disease propagation, leading to a computational complexity of $O\left(N^{2}\right)$ equations in the worst case.

Compared to these approaches, the moment expansion approach discussed above and moment closure using, e.g., the $\mathrm{PA}$, have the advantage of yielding simple, low- 


\section{Simulation and analysis of adaptive networks}

dimensional ODE systems, which can be analysed with the tools from dynamical systems theory and thus reveal the mechanisms driving the model dynamics. Depending on the particular application, however, the accuracy of the $\mathrm{PA}$ and similar closures may not be satisfactory, so that more fine-grained methods are required. 


\section{Swarming behaviour}

In this chapter, I develop an adaptive-network approach to the phenomenon of swarming, i.e. collective coherent motion in large groups of social animals. I show how the onset of collective motion can be understood from the adaptive nature of the interaction network among the swarm members.

Among the large number of collective phenomena in nature, animal swarms certainly belong to the most impressive ones. The elegance of bird flocks and fish schools, the power of buffalo herds, or the destructive force of locust swarms have always fascinated humans throughout history.

But swarms ${ }^{1}$ are not only interesting because of their mesmerizing nature. As a swarm, many animals can solve complex tasks far beyond what a single individual would be able to achieve. A single ant, for instance, is a relatively simple insect (if one neglects the complexity of biochemical processes responsible for its every-day life), and yet a colony of hundreds of thousands of them builds a highly sophisticated nest structure containing an intricate system of underground passages and chambers. Furthermore, recent studies show that many animals can often make better choices in groups than individually (Couzin et al., 2005, Couzin, 2007. Cucker and Huepe, 2008). Swarming behaviour seems thus to allow for effective collective action, a phenomenon which has been termed swarm intelligence. As a collective phenomenon, it appears to follow from universal organizing principles (Bonabeau et al. 1999).

In the following, I focus on the basic phenomenon of collective motion in animal swarms, that is, how coherent motion is achieved in a large group of animals without central control or a clear leader. I discuss the onset of collective motion as a dynamical phenomenon, which is traditionally modelled using agent-based simulations of self-propelled particles (SPP). After giving an introduction to a paradigmatic such agent-based model, I present an adaptive-network approach to a specific experiment focusing on the onset of collective motion in desert locusts.

\subsection{Collective motion in swarms}

Depending on the particular species, insect swarms may comprise from a few hundred up to millions of individuals. A small number of insects, however, runs about incoherently instead of forming a cohesive swarm. 'A hundred army ants put together will walk in meaningless circles until they die' (Franks, 1989, Mitchell, 2009). Yet, half a million of them can form an intimidating "super-organism" raiding rainforest grounds and devouring

${ }^{1}$ In the following, I refer to flocks, herds, schools, etc. by the general term swarm. 
everything edible on its way. This is not only true for insect swarms. Coherent collective behaviour seems to emerge in general only in sufficiently large animal groups. Understanding the onset of swarming behaviour, i.e. the transition from incoherent, disordered animal groups to coherent swarms of collectively moving animals, is a key challenge in the research on animal swarming and a prerequisite for the development of efficient approaches to controlling animal swarms.

\subsubsection{Models of collective motion}

In the research on collective motion, essentially two different modelling approaches have been pursued so far. In the agent-based approach, the focus is on the motion of the individual swarm members, which are often modelled as self-propelled particles (SPP $)$ (Vicsek et al., 1995, Mach and Schweitzer. 2007, Aldana et al., 2007, Chaté et al. 2008, Huepe and Aldana, 2008. Sumpter et al. 2008. Romanczuk et al. 2009). By contrast, in fluid-like models, one considers the whole swarm as a cohesive fluid that can be studied using concepts from hydrodynamics (Toner and Tu, 1995, 1998: Topaz and Bertozzi 2004). In the present chapter, I present a complementary approach focusing on the adaptive interaction network among the individual swarm members. It is developed starting from an agent-based viewpoint. Therefore, I briefly discuss the most influential agent-based model, the Vicsek model, but I pay no further attention to the hydrodynamic approach.

The most famous agent-based model, which has become the paradigm of agent-based models for collective motion, was proposed by Vicsek et al. (1995). In this simple twodimensional model, a swarm of SPP, is considered, all of which move at the same constant speed, i.e. absolute value of their velocity. They seek to align their direction of motion to the average direction of the neighbouring particles within an interaction radius $r$. Specifically, the position $\mathbf{x}_{i}$ and direction of motion $\theta_{i}$ of the $i$ th particle are updated simultaneously according to

$$
\begin{aligned}
& \mathbf{x}_{i}(t+\Delta t)=\mathbf{x}_{i}(t)+\mathbf{v}_{i}(t) \Delta t, \\
& \theta_{i}(t+\Delta t)=\left\langle\theta_{i}(t)\right\rangle_{r}+\xi_{i},
\end{aligned}
$$

where $\left\langle\theta_{i}(t)\right\rangle_{r}$ denotes the average direction of the velocities of the $i$ th particle and all neighbouring particles within the radius $r$, and $\xi_{i}$ is a noise term drawn uniformly at random from $[-\eta / 2, \eta / 2]$ for each particle. Only the particles' direction of motion is updated, while the absolute value of their velocity is held fixed, so that $\left\|\mathbf{v}_{i}\right\|=v$ for all $i$. Hence, the particles tend to align to their neighbours but at the same time are subject to fluctuations, whose intensity range can be controlled by varying $\eta \in[0,2 \pi]$. For $\eta=0$, the particles always align to the average direction of motion within their interaction radius, which gives rise to purely deterministic dynamics. For $\eta=2 \pi$, on the other hand, the particles do not interact with their neighbours but choose a random direction on each update, which gives rise to purely random motion. The parameter $\eta$ thus plays a similar role in this system as temperature in equilibrium many-particle systems. 
In simulations of this simple model, a non-equilibrium phase transition is observed between a disordered phase and an ordered phase when the noise intensity $\eta$ is decreased. At high noise intensity or, equivalently, low particle density, the particles move in an uncorrelated fashion without any preferred direction. At low noise intensity, the particles align and tend to move in the same, spontaneously selected direction, thus breaking the rotational symmetry. This transition is clearly visible in the behaviour of a characteristic order parameter, namely the normalized average velocity $\bar{v}=\left\|\sum_{i} \mathbf{v}_{i}\right\| / N v$. In the disordered phase $\bar{v}=0$, whereas $\bar{v}>0$ in the ordered phase.

The observed phase transition from a disordered, incoherent state to ordered, collective motion is often referred to as the swarming transition. Although a non-equilibrium phase transition, it shows similar characteristics as well-known equilibrium phase transitions, such as the order-disorder transition in the two-dimensional Ising model, for instance. In particular, characteristic scaling behaviour of the order parameter $\bar{v}$ is observed close to the critical point, indicating that the transition is of second order.

Several other SPP models have been considered, ranging from simple variations of the Vicsek model to more sophisticated models based on, e.g., phenomenological particle interaction potentials (Grégoire and Chaté, 2004, Mach and Schweitzer. 2007, Chaté et al. 2008: Cucker and Huepe. 2008. Strefler et al. 2008. Dossetti et al. 2009). In all of them, a swarming transition from disordered to ordered collective motion is observed, indicating that this is a robust emergent phenomenon. In so-called zonal models, for instance, three layered interaction zones are defined either explicitly or by an appropriate choice of an interaction potential: to avoid collisions, agents try to move away from agents within a short-range repulsion zone, they align to agents within an intermediaterange alignment zone, and they move towards agents within a long-range cohesion zone (Lukeman et al. 2010). Thus, cohesive swarms are obtained in simulations, whose visual appearance resembles real bird flocks, fish shoals, or insect swarms. The three basic ingredients-separation, alignment, and cohesion - were already introduced in the first SPP computer simulation of swarms (Reynolds 1987), and have since then been used in animation algorithms for films and computer games.

Coherent collective motion is achieved in the SPP models, because the local interactions among the particles give rise to emergent long-range order. This is made possible by the motion of the particles, which, over time, builds up long-range correlations in their moving directions as they enter and leave their respective interaction ranges. Thus, the particle motility generates effective long-range interactions, although the microscopic rules are purely local (Toner and Tu, 1995).

In fact, Aldana and Huepe (2003) suggested that the existence of the phase transition does not depend on the details of the kinematic updating rule as long as it generates longrange interactions. To show this, they used a network approach in which they considered non-moving particles placed on a regular lattice and aligning to their lattice neighbours, similar to the two-dimensional XY-model. There is no transition to an ordered regime in the regular lattice, but the transition from disorder to order is observed as soon as the lattice is transformed into a small-world network by randomly introducing a small 


\section{Swarming behaviour}

number of long-range links.

While the existence of the phase transition seems to depend only on the presence of long-range interactions and not on the model details, its order appears to be determined by the specific kinematic interaction rules. In particular, it has been shown that whether the transition is continuous or discontinuous depends crucially on how noise is incorporated in the system (Grégoire and Chaté 2004, Aldana et al., 2007, Eftimie et al. 2007, Nagy et al. 2007. Chaté et al., 2008 Aldana et al. 2009).

Aldana and Huepe (2003) and Aldana et al. (2007) already suggested to use complex networks to model the interactions among SPP. Their studies focused on the effect of long-range connections in static interaction networks and did not consider the changes in the interaction network that would be caused by the particle motion. In the following, I present an adaptive-network model of swarm dynamics, in which these motion-driven network changes are taken into account explicitly. This model is based on a recent experiment investigating the swarming behaviour of juvenile desert locusts.

\subsubsection{A swarming experiment}

In a recent experiment, the swarming behaviour of the desert locust, Schistocerca gregaria, has been investigated (Buhl et al. 2006). This species is known to exhibit densitydependent polyphenism. When many locusts are kept in a crowd, their repeated, accidental touching each other's back legs stimulates them to switch from a "solitarious" phase to a "gregarious" phase, which differs in behaviour and physiology (Simpson et al. 1999. 2001. Anstey et al. 2009). In the solitarious phase, the insects are shy and non-social, whereas they are inclined to aggregate in large numbers in the gregarious phase. Before taking flight as adults, wingless juvenile insects (nymphs) in the gregarious phase form large marching groups that can extend over kilometres. The swarming transition is thus related to the change from the solitarious to the gregarious phase in the locust nymphs. Buhl et al. studied its dependence on the insect density.

In the experiment, up to 120 locust nymphs were placed in a ring-shaped arena and left to march freely for eight hours, while their positions and orientations were tracked using a digital camera. The insects walk around in the arena and react on the encounters with others by changes in direction and/or walking speed. These local interactions seem to be driven by the insects' tendency towards cannibalism (Simpson et al. 2006, Bazazi et al. 2008), leading to dynamics of "pursuit and escape" among the individuals (Romanczuk et al. 2009). Buhl et al. observed how this gives rise to ordered collective motion.

From the orientations of the locusts, an order parameter was computed, namely the locusts' instantaneous alignment,

$$
\Phi(t)=\frac{2}{m \pi} \sum_{i}^{m} \chi_{i}(t)
$$

Here, $m$ is the number of moving locusts, and $\chi_{i}(t)$ is the orientation of the $i$ th insect, defined as the smallest angle between two consecutive insect positions and a line drawn 

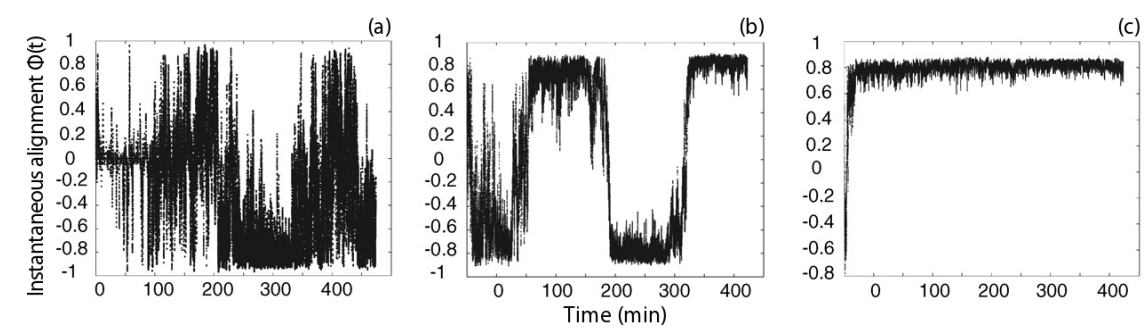

Figure 4.1.: Instantaneous alignment $\Phi(t)$ for 7 (a), 20 (b), and 60 (c) locusts in the arena. At low insect number, no ordered collective motion is observed. Persistent coherent motion arises at high insect number, while an intermittent switching regime is observed at intermediate numbers. Figure adapted from (Buhl et al., 2006).

from the centre of the arena to the first position. The order parameter takes its extreme value \pm 1 when all insects are aligned in the same direction, whereas a value of $\Phi(t)=0$ indicates a disordered state.

In Fig. 4.1. time series of the measured order parameter are shown for different numbers of insects, which correspond to insect densities due to the fixed geometry. At a low density, $\Phi(t)$ fluctuates strongly but no ordered collective motion arises. The locusts walk around incoherently in the arena. At an intermediate density, the locusts start aligning, and periods of collective rotational motion are observed, in which most of the insects march in the same direction. These periods are interrupted by rapid spontaneous direction changes, switching the collective heading from clockwise to counter-clockwise or vice versa. At a high density, no such spontaneous direction switches are observed, and the locusts adopt a persistent common marching direction.

With increasing insect density, a swarming transition is thus observed in the experiment, going from disordered to ordered collective motion via an intermediate region of intermittent switching between the two marching directions. Buhl et al. were able to reproduce these experimental results qualitatively in simulations of a modified onedimensional Vicsek model similar to (4.1). Subsequent studies concentrated on the role of noise in this system, suggesting that inherent noise plays a key role in establishing and maintaining coherent motion (Yates et al. 2009: Escudero et al. 2010).

In the next section, I present an alternative modelling approach for the locust experiment, which focuses on the adaptive interaction network among the insects instead of considering explicitly the motion of each insect in a system of SPP. Inspired by previous work on opinion formation in populations of interacting agents, it reveals the similarities between swarming and collective decision-making. 


\subsection{Adaptive-network approach to swarming ${ }^{2}$}

Swarming behaviour and collective motion have many similarities with collective decisionmaking processes, as for instance collective opinion formation in humans (Conradt and List. 2009). Indeed, cohesive swarms, in which all individuals move in the same direction, can only form if some consensus about this direction is reached among the individuals. In a swarm, such consensus seems to be achieved by an effective long-range communication mediated by the local interactions.

It is interesting to note that, although regarded as collective decision-making processes, swarming behaviour and collective motion are modelled differently from decision making in human populations. Theoretical studies of collective motion have mostly focused on agent-based simulations of $\mathrm{SPP} \beta$ and fluid-like models that treat the whole swarm as a continuous medium. By contrast, studies of decision making and opinion formation in social systems typically represent the system as a network, emphasizing the discrete nature of interactions (Holley and Liggett, 1975, Liggett 1999 . Sood and Redner. 2005. Castellano et al. 2009). Although a network approach was used to investigate the role of long-range interactions in the swarming transition (Aldana and Huepe, 2003: Aldana et al., 2007), the relation to network models of opinion formation has not yet been considered. One of the reasons for this difference in modelling approaches is that for swarm systems, spatial embedding is assumed to be of central importance, whereas social interactions are felt to be less constrained by physical space.

Furthermore, in collective motion, it cannot be neglected that an agent's decision to move in a certain direction determines the agents with whom it will be interacting next. This feedback of individual decisions on future interaction partners is, however, reminiscent of the feedback of the local dynamics on the topological evolution in adaptive networks. Indeed, the moving agents can be considered as network nodes, whose node states correspond to the agents' moving directions. Two nodes are linked if the corresponding agents can interact with each other, i.e. if they are close enough to each other in the underlying space. As the agents move, links are created and destroyed depending on the agents' direction of motion, i.e. the node states. Thus, the topological evolution of the agents' interaction network is affected by their local dynamics, rendering the interaction network an adaptive network.

Starting from the insight that the spatial embedding leads to an adaptive interaction network, we propose in the following a non-spatial adaptive-network model of swarming behaviour, in which spatial information is used only as a rationale for the network adaptivity, while any explicit spatial embedding is neglected. Reproducing characteristic observations for swarm systems, we find that spatial geometry might play a less central role than has been assumed. Our approach highlights the analogy between swarming and opinion formation on adaptive networks (Holme and Newman, 2006: Gil and Zanette,

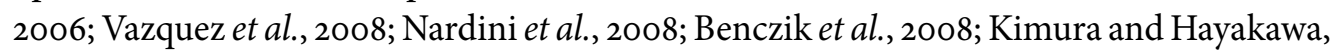

${ }^{2}$ This section is based on the author's article (Huepe et al. 2011) published in the New Journal of Physics. 


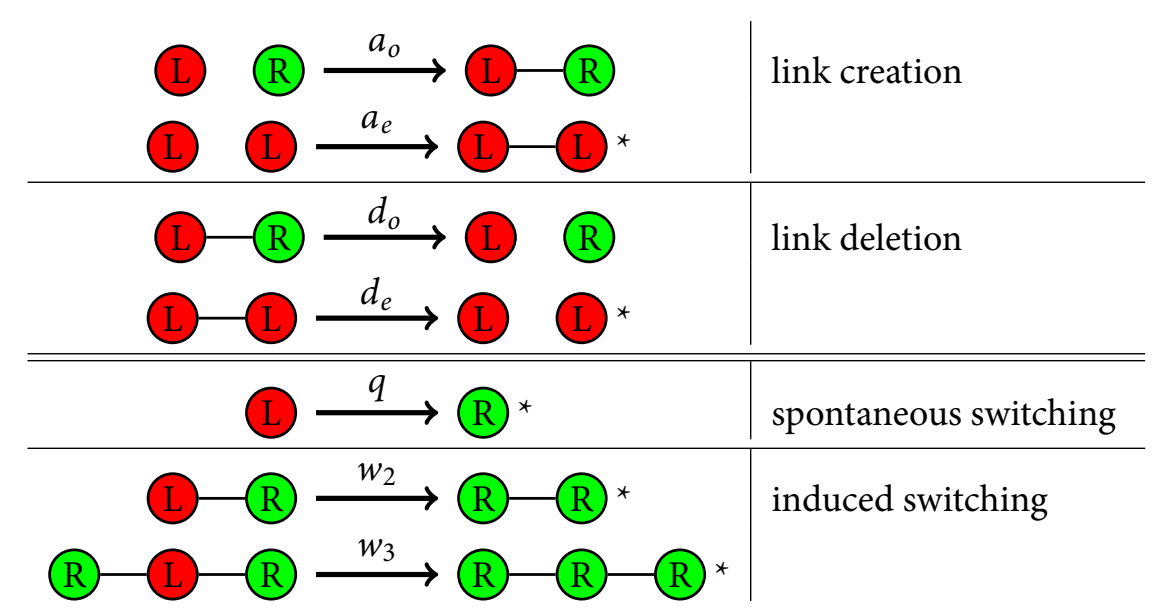

Table 4.1.: Transformation rules used to define the adaptive-network model of the swarming experiment. For starred $\left(^{*}\right)$ rules, the rule obtained by swapping $\mathrm{R}$ and $\mathrm{L}$ is also present in the model definition.

2008 Böhme and Gross 2011), thus building a bridge between two areas of research that have been so far considered separately.

\subsubsection{Model}

We focus on the experiment by Buhl et al. (2006) discussed in section 4.1.2. Instead of recreating this experiment in silico by using a Vicsek-type model of SPP using a different approach. We try to address the mechanism leading to the observed collective dynamics with the help of a simple low-dimensional description that lends itself to analytical treatment. To that end, we consider the system of interacting agents as an adaptive network of $N$ nodes, representing the locusts. Nodes are linked if the corresponding locusts are mutually aware of each other through any interaction mechanism. As in (Buhl et al. 2006), we distinguish only two directions of motion, so that the possible node states are chosen from the set $\mathcal{S}=\{\mathrm{R}, \mathrm{L}\}$, where a node in state $\mathrm{R}$ or $\mathrm{L}$ represents an agent that marches clockwise (a right-goer) or counter-clockwise (a left-goer), respectively. We refer below to pairs of nodes in the same state as equal-goers and to those in different states as opposite-goers.

The proposed model only takes into account the agents' headings and contact network while neglecting all other information, including insect positions. The evolution of the network is modelled by the transformation rules in Tab. 4.1 As locusts advance in the experimental system, non-interacting opposite-goers eventually meet and start sensing each other, as schematically shown in Fig. 4.2 a. We model this by randomly introducing R-L-links at a rate $a_{o}$ per node. In addition, interacting opposite-goers will eventually lose contact, which is modelled by the random deletion of R-L-links at a rate $d_{o}$ per link (Fig. 4.2 d). Likewise, equal-goers can start or stop interacting as they approach 


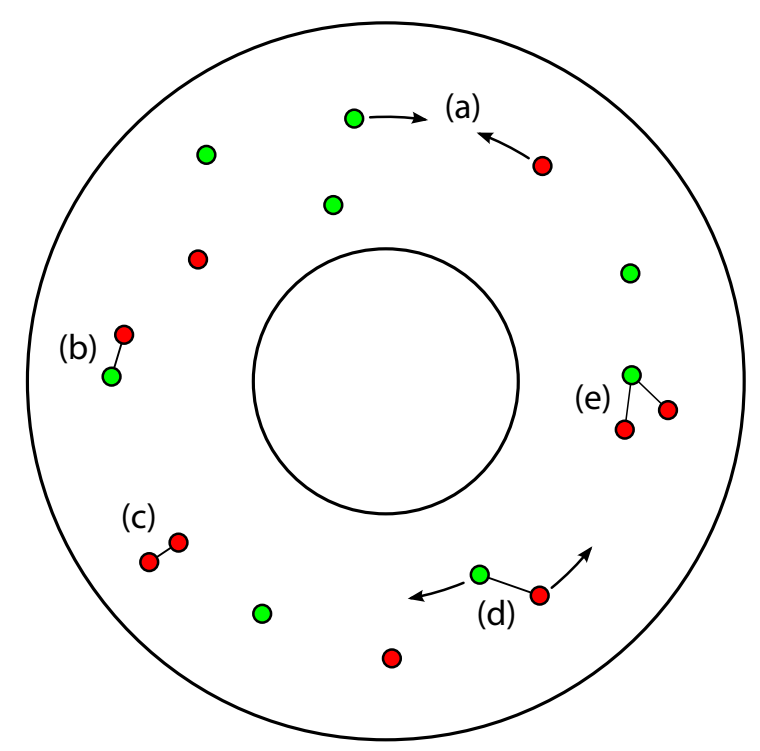

Figure 4.2.: Schematic illustration of the interaction processes in the adaptive-network model, depicted in the arena geometry of the original experiment: (a) upon encounter, left-going (red) and right-going (green) agents create a link between them; (b) linked opposite-goers can interact; (c) linked equal-goers may also interact; (d) linked agents can dissociate; (e) agents can interact with more than one other agent.

or separate from each other due to marching speed differences or lateral displacements (Fig. $4.2 \mathrm{c}$ ). This is represented by also introducing for equal-goers the attachment rate per node $a_{e}$ and deletion rate per link $d_{e}$. Using these conventions, all rates are defined as intensive quantities.

The state dynamics of each node is given by a stochastic process that depends on its topological neighbours. It is reasonable to assume that the probability for an agent to switch direction is some non-linear function of its number of adjacent opposite-goers. Instead of explicitly modelling this in terms of the general transformation rule

$$
\mathrm{L}_{i} \stackrel{g\left(\mathcal{N}_{i}\right)}{\longrightarrow} \mathrm{R}_{i}
$$

with a non-linear transition rate $g\left(\mathcal{N}_{i}\right)$, we consider three linear transformation rules acting on different $\mathrm{LHS}$ motifs. Taken together, these are expected to generate the same macroscopic behaviour as the corresponding non-linear rule (cf. appendix A). We assume that each node switches direction with probability $w_{2}$ for every R-L-link it has to an opposite-goer (Fig. 4.2 b). This corresponds to a symmetric CP. in which L-nodes can "infect" R-nodes, and vice versa. Additionally, we introduce a probability $w_{3}$ of the central node switching direction for every L-R-L- and R-L-R-chain (Fig. 4.2 e), which accounts for non-linear three-agent interactions. Finally, noise is represented by a constant probability $q$ of an agent spontaneously switching direction. 
All transformation rules are implemented using the motif update scheme: when applying a rule, the LHS subgraph is selected uniformly at random from all the corresponding subgraphs in the network rather than being "discovered" by successively selecting a random node and its neighbours (cf. section 3.1.2).

\subsubsection{Analytical description}

Following the moment expansion approach in 3.3 . we derive a system of ODE; for the network moments. The zeroth-order equations for the densities of left and right-goers can be straightforwardly derived as

$$
\begin{aligned}
\frac{\mathrm{d}}{\mathrm{dt}}[\mathrm{R}] & =\sum_{\sigma_{i}=\mathrm{L}} \frac{q}{N}-\sum_{\sigma_{i}=\mathrm{R}} \frac{q}{N}+\sum_{\sigma_{i j}=\mathrm{LR}} \frac{w_{2}}{N}-\sum_{\sigma_{i j}=\mathrm{LR}} \frac{w_{2}}{N}+\sum_{\sigma_{i j k}=\mathrm{RLR}} \frac{w_{3}}{N}-\sum_{\sigma_{i j k}=\mathrm{LRL}} \frac{w_{3}}{N} \\
& =q([\mathrm{~L}]-[\mathrm{R}])+w_{3}([\mathrm{RLR}]-[\mathrm{LRL}]),
\end{aligned}
$$

and the symmetric expression for $[\mathrm{L}]$, obtained by interchanging $\mathrm{R}$ and $\mathrm{L}$. Due to the symmetry between left- and right-goers in our model, the $w_{2}$-process does not appear in the zeroth-order equations. The first-order equations are

$$
\begin{aligned}
\frac{\mathrm{d}}{\mathrm{dt}}[\mathrm{RR}]=q([\mathrm{LR}]-2[\mathrm{RR}])+w_{2}([\mathrm{LR}]+2[\mathrm{RLR}]-[\mathrm{RRL}]) \\
\\
+w_{3}\left(2[\mathrm{RLR}]+3\left[{ }^{\mathrm{R}} \mathrm{L}_{\mathrm{R}}^{\mathrm{R}}\right]-\left[{ }^{\mathrm{R}} \mathrm{R}_{\mathrm{L}}^{\mathrm{L}}\right]\right)+a_{e}[\mathrm{R}]{ }^{2}-d_{e}[\mathrm{RR}]
\end{aligned}
$$

and the symmetric expression for $[\mathrm{LL}]$. Here we use $\left[{ }^{A} B_{D}^{C}\right]$ to denote the density of third-order motifs with a central node in state $B \in\{\mathrm{L}, \mathrm{R}\}$ linked to three nodes in states $A, C, D \in\{\mathrm{L}, \mathrm{R}\}$. Such third-order motifs appear in the equations due to the three-body $w_{3}$-process. Similarly to the $\mathrm{CP}$, this process leads to a cascade of moment equations, in which moments of order $n+1$ and $n+2$ are involved in the evolution equations for moments of order $n$.

Finally, rather than writing an equation for [LR], we note that the total first-order moment dynamics depends only on the link creation and deletion processes through

$$
\begin{aligned}
\frac{\mathrm{d}}{\mathrm{dt}}([\mathrm{LR}]+[\mathrm{RR}]+[\mathrm{LL}])=a_{o}[\mathrm{~L}][\mathrm{R}] & -d_{o}[\mathrm{LR}] \\
& +a_{e}\left([\mathrm{R}]^{2}+[\mathrm{L}]^{2}\right)-d_{e}([\mathrm{LL}]+[\mathrm{RR}])
\end{aligned}
$$

This system of ODE for the zeroth-order and first-order moments is not yet closed, as it involves higher-order moments. In order to close it, we approximate the triplets and quadruplets using the $\mathrm{PA}$ as

$$
[\mathrm{RLR}] \approx \kappa \frac{[\mathrm{LR}]^{2}}{2[\mathrm{~L}]}, \quad[\mathrm{RRL}] \approx 2 \kappa \frac{[\mathrm{LR}][\mathrm{RR}]}{[\mathrm{R}]},
$$




$$
\left[{ }^{\mathrm{R}} \mathrm{L}_{\mathrm{R}}^{\mathrm{R}}\right] \approx \kappa^{2} \frac{[\mathrm{LR}]^{3}}{6[\mathrm{~L}]^{2}}, \quad\left[{ }^{\mathrm{R}} \mathrm{R}_{\mathrm{L}}^{\mathrm{L}}\right] \approx \kappa^{2} \frac{[\mathrm{LR}]^{2}[\mathrm{RR}]}{[\mathrm{R}]^{2}},
$$

and symmetric expressions. As for the second-order moments, we assume that the $A$-, $C$-, and $D$-nodes in $\left[{ }^{A} B_{D}^{C}\right]$ are uncorrelated in this approximation.

The factor $\kappa=\langle q\rangle /\langle k\rangle$ relates the second and first moments of the degree distribution. Because links are randomly created and destroyed in our model all the time, we expect not too large deviations from the initial Poisson distribution in the degree distribution of the unknown, randomly evolving network topology. We thus set $\kappa=1$, which is its exact value for the Poisson distribution (cf.2.2). This approximation has been routinely used in related models of opinion formation and epidemic spreading in (adaptive) networks (Keeling et al. 1997 Gross et al. 2006. Vazquez et al. 2008 Kimura and Hayakawa 2008).

For $a_{e}=d_{e}=0$, the stationary solutions of the ODE system (4.3)-(4.5) decouple, with Eqs. (4.3) and (4.5) solved independently. In this case, we obtain analytically a mixed-phase solution branch

$$
[\mathrm{R}]^{*}=[\mathrm{L}]^{*}=\frac{1}{2}
$$

which becomes unstable in a supercritical pitchfork bifurcation (cf. appendix B) at $a_{o}^{*}=$ $2 d_{o} \sqrt{2 q /\left(\kappa w_{3}\right)}$. At this point, two stable solution branches appear,

$$
[\mathrm{R}]_{ \pm}=\frac{1}{2} \pm \frac{1}{2} \sqrt{1-\frac{8 q d_{o}^{2}}{\kappa w_{3} a_{o}^{2}}} .
$$

These stationary states are asymmetric states, in which a majority of the nodes is in the same state (either $\mathrm{L}$ or $\mathrm{R}$ ).

For $a_{e} \neq 0$ and $d_{e} \neq 0$, the stationary solutions can be computed numerically by solving the corresponding system of algebraic equations. In this case, we also find a supercritical pitchfork bifurcation for small $a_{e}$, as shown in Fig. 4.3 (top). For higher values of $a_{e}$, however, the transition occurs through a subcritical pitchfork bifurcation (Fig. 4.4, rightcolumn insets). This yields a bistable phase where ordered and disordered states coexist, highlighted in Fig. 4.3 (bottom).

Note that the pitchfork bifurcation is only obtained if non-linear interactions involving triple motifs are present in the system, i.e. $w_{3}>0$. This is true both for $a_{e}=d_{e}=0$ and $a_{e}, d_{e} \neq 0$. Non-linearity, as introduced by the three-body interactions in our model, is thus an essential ingredient to break the symmetry and allow for ordered states.

We note that a supercritical pitchfork bifurcation, in which the stable solution branches are given by

$$
[\mathrm{R}]_{ \pm}^{0}=\frac{1}{2} \pm \frac{1}{2} \sqrt{1-\frac{4 q}{w_{3}}},
$$

could be already observed in a mean-field approximation, in which the system is closed at the zeroth order by approximating the third-order moments in equation 4.3 in terms of the node densities. However, the PA is more accurate when compared with stochastic 


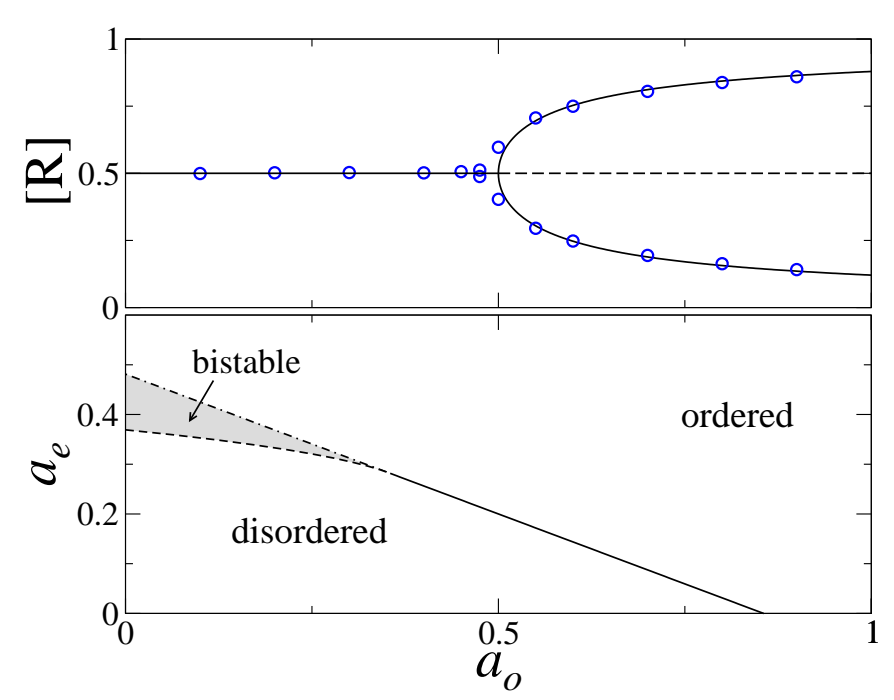

Figure 4.3.: Top: Bifurcation diagram of the density of right-goers [R] vs. link creation rate $a_{o}$. Solutions of the ODE system (solid line) yield a supercritical pitchfork bifurcation in excellent agreement with results from network simulations (circles). Bottom: Phase diagram showing the bifurcation point as a function of the link creation rates $a_{o}$ and $a_{e}$. In the bistable region (grey), the pitchfork bifurcation becomes subcritical. Parameters: $N=10^{4}, d_{o}=0.25, d_{e}=0.1$, $w_{2}=w_{3}=0.2, q=0.1$, and (top only) $a_{e}=0.2$.

simulations of the network. Moreover, it allows for the new class of subcritical solutions, which we discuss below.

\subsubsection{Results}

In Fig. 4.3 (top), we show that the $\mathrm{ODE}$ system solutions are in excellent agreement with individual-based stochastic simulations of the network dynamics. Varying the linkcreation rate $a_{o}$, we observe a pitchfork bifurcation according to 4.7 from a mixed state, where $[\mathrm{R}]^{*}=1 / 2$, to an asymmetric state, where one of the two node states $\mathrm{L}$ or $\mathrm{R}$ is in the majority. We verified that the small remaining discrepancy is due to the assumption that $\kappa=1$, and not other factors such as finite-size effects.

We now compare the results of our adaptive-network model to the locust experiments by Buhl et al. (2006) (Fig. 4.1). The bifurcation diagram in Fig. 4.3 uses the link-creation rate between opposite-goers $a_{o}$ as control parameter, which is proportional to the experimental agent density in our current framework ${ }^{3}$ We can thus compare our results directly to the behaviour observed experimentally at different agent numbers.

For a low association rate $a_{o}$, the network is found in a mixed state with $[\mathrm{R}]^{*}=1 / 2$,

${ }^{3}$ Assuming a homogeneous insect distribution in the arena and an equal, constant angular velocity $\omega$ for each insect (clockwise or counter-clockwise), it is straightforward to derive $a_{o}=(\omega / \pi) N$ from the average encounter rates between left- and right-going individuals. 


\section{Swarming behaviour}

exhibiting no order or symmetry breaking. This corresponds to the disordered mix of left- and right-goers obtained in experiments at low insect number, in which no coherent collective motion develops.

For high $a_{o}$, the system is driven towards one of the two stable solution branches, with a majority of nodes in either state ( $\mathrm{R}$ or L). This is the ordered collective marching state found at high insect number, in which the majority of the insects walks collectively either clockwise or counter-clockwise. A similar pitchfork bifurcation is also observed when using the noise intensity, $q$, instead of $a_{o}$ as control parameter, as done in most previous numerical works (Vicsek et al. 1995. Chaté et al. 2008. Huepe and Aldana 2008). In this case, the disordered state is observed for large $q$, and the ordered states for small $q$.

Let us emphasize that the presence of this transition in the adaptive-network model implies that very few elements of the agent dynamics are required to obtain such swarming behaviour. In particular, we did not choose any specific interaction rule but only required that it drives agents to head in the same direction. By contrast, we find that three-body, i.e. non-linear, interaction processes are required to break the symmetry and obtain swarming solutions. Furthermore, a subcritical bifurcation, giving rise to hysteresis or sudden polarization, is only possible in the adaptive-network model if $a_{e}$ and $d_{e}$ are non-zero. This qualitative result could shed light on the current controversy over the order of the swarming transition (Vicsek et al. 1995. Aldana et al. 2007. Nagy et al. 2007, Chaté et al. 2008 Pimentel et al. 2008). Indeed, a first-order transition (stemming from the subcritical pitchfork bifurcation) is only possible here if groups of equal-goers can associate or dissociate while heading in the same direction. It would be very interesting to explore if a similar effect is present in agent-based simulations and experiments.

Fig. 4.4 shows the degree distributions obtained in the ordered and disordered phases for supercritical and subcritical cases. Both display similar connectivities. In the disordered states (top), most agents have very few links to equal-goers and no links to opposite-goers. This is also observed in agent-based simulations and experiments (Buhl et al. 2006. Huepe and Aldana 2008), where the disordered regime develops no large clusters and, therefore, small connectivity.

In the ordered (right-going) state (bottom), R-R links are strongly favoured. This corresponds to the formation of large right-going groups in the agent-based dynamics. The number of L-R links also increases, which corresponds to encounters between a few left-goers and these large right-going clusters. The typical number of all other links decreases.

In previous experiments and simulations it was observed that there is an intermittent regime at intermediate insect densities, where the swarm is polarized but can switch the marching direction spontaneously (Fig. $4.1 \mathrm{~b}$ ). This behaviour is also captured by the adaptive-network model. The intermittent regime occurs in simulations of the network at $a_{o}$-values in the ordered phase close to the bifurcation, where a low nucleation barrier allows for stochastic switching between the two branches.

Fig. 4.5 shows the survival probability of ordered states, i.e. the cumulative distribution of residence times (lasting $\tau$ or longer) in which the network resides in a majority $\mathrm{R}$ 

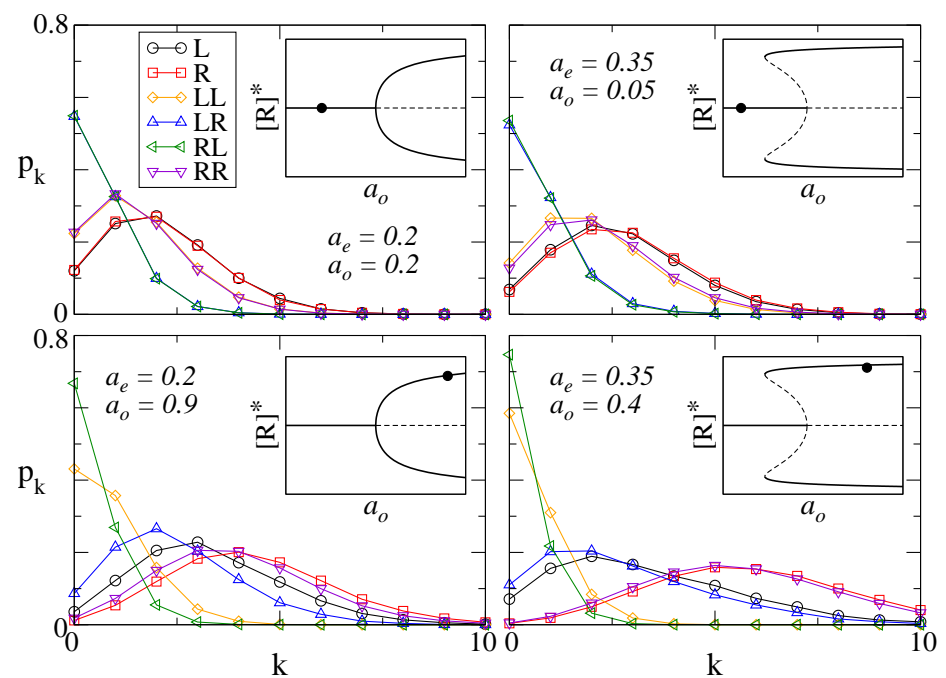

Figure 4.4.: Degree distributions of four different stationary solutions obtained through adaptive network simulations. The top (bottom) row shows cases in the disordered (ordered) phase, with insets displaying their location in the bifurcation diagram. The left (right) column shows cases with a supercritical (subcritical) pitchfork bifurcation. Different curves display the connectivity of left-goers (L), of right-goers (R), of left-goers only to left-goers (LL) or only to right-goers (LR), etc. Parameters: Same as in Fig. 4.3 except when noted on plots.

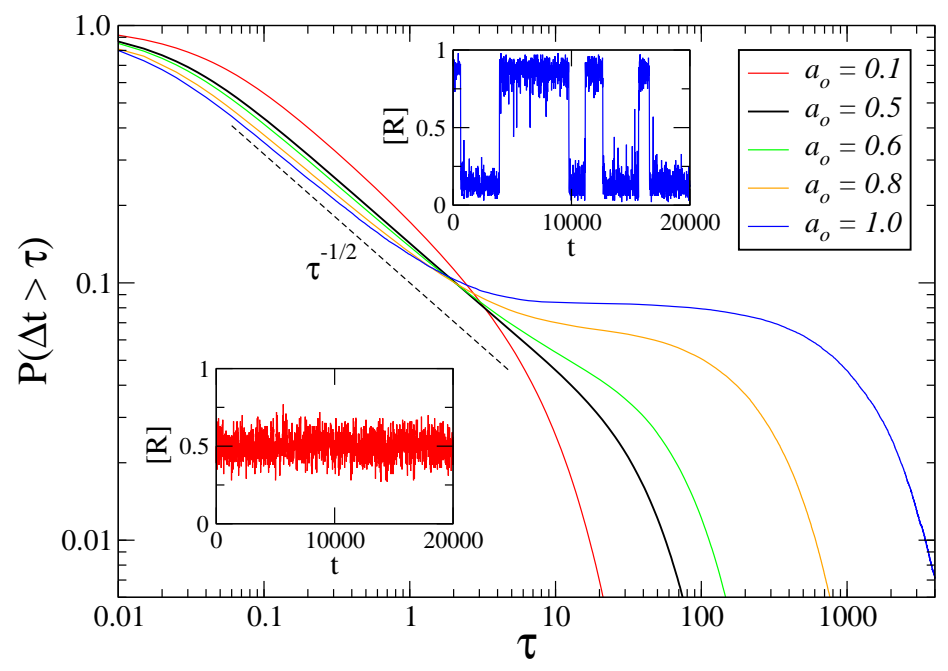

Figure 4.5.: Cumulative distribution of residence-times in a majority $\mathrm{L}$ or majority $\mathrm{R}$ state for the adaptive network dynamics at various values of $a_{0}$. As $a_{o}$ is increased, the system becomes more ordered, switching direction less often, and the distribution becomes broader. For $a_{0}>0.6$, a preferred residence time appears at large $\tau \sim 10^{3}$ due to finite-size effects. Insets show the density of right-goers [R] vs. time for $a_{o}=0.1$ (bottom-left) and $a_{o}=1.0$ (top-centre). Parameters: Same as in Fig. $4 \cdot 3$ (top) but for $N=100$ nodes. 


\section{Swarming behaviour}

state before switching to a majority L state or vice-versa. In the disordered phase, this distribution decays exponentially as expected, since it results from memoryless stochastic fluctuations about the stationary state (van Kampen, 1997). As $a_{o}$ approaches its critical value, the distribution develops a long tail approximating a power law with exponent $-1 / 2$, providing evidence for a switching process with memory similar to the one observed in intermittent turbulent hydrodynamic flows (Bramwell et al. 1998 , Portelli et al. 2003). We note that a power-law distribution of switching times with the same exponent was observed in previous spatial SPP models (Huepe and Aldana 2004, 2008). The experimental locust dynamics also display intermittency at intermediate densities, but the available time series are not long enough to characterize its statistics.

For $a_{o} \geq 0.8$ finite-size effects produce a preferred residence time at large $\tau \sim 10^{3}$ that grows with the system size, appearing as a plateau in the cumulative distributions. This corresponds to the typical escape time from highly polarized states, where the system gets trapped when system-wide connectivity is reached within the finite network.

\subsubsection{Conclusions}

In summary, we have proposed an adaptive-network model of a swarm experiment that captures much of its characteristic collective behaviour and likens it to an opinionformation process. In particular, our model displays a transition from a disordered to an ordered phase with increasing insect density. Furthermore, an intermittent regime is observed close to the transition point, where a fat-tailed distribution of residence-times emerges.

We emphasize that in the proposed model, these characteristics of swarming systems are recovered without an explicit spatial representation of the system. This suggests that the spatial context of swarming may not be of central importance for many phenomena. By contrast, non-linear interactions (as implemented by the three-body processes) and an increased probability of interaction between agents with intersecting trajectories are found to be essential in our model.

Further work is certainly necessary to test whether the experimentally observed transition is caused by the same mechanism that is at work in our non-spatial model. In order to improve our understanding of the role of spatial dynamics in swarms, detailed network- and agent-based simulations should therefore be compared. We postulate that the same dynamics will be followed by point-like agents, which are well described by the nodes in our model, but not by spatially extended agents, where jamming must play an important role.

In the present work, we used a modelling approach originally proposed for social networks. We believe that the analogies we have drawn between swarming phenomena and opinion formation processes could be fruitfully exploited in further studies, thus building a bridge between the two fields. 


\subsection{Summary}

In this chapter, we have applied the concept of adaptive networks to the modelling of swarming behaviour in animals. In particular, a novel modelling approach for the collective motion in swarms was proposed, in which the network formed by the interactions among the swarm members is considered instead of modelling the spatial dynamics. We have seen that the characteristic swarming transition from disordered to ordered collective motion as observed in a recent experiment with desert locusts can be captured by a minimal adaptive-network model without an explicit spatial representation of the moving agents. In our model, the agents interact in an abstract adaptive network, where the effects of spatial embedding, insect motion, and interaction are mimicked by stochastic transformation rules.

Using this approach, it is possible to run simulations of very large systems at relatively low computational cost, which is often difficult with traditional agent-based simulations of SPP: Moreover, we have derived from the microscopic transformation rules a macroscopic description in terms of a low-dimensional system of ODE, which allows for the detailed analysis of the emergent behaviour in the framework of dynamical systems theory.

In our model, the swarming transition is reproduced as a sub- or supercritical pitchfork bifurcation, in which the disordered stationary state becomes unstable. We have seen that this bifurcation is caused by the adaptive nature of the network, as it is only present when the coupling between local and topological degrees of freedom is switched on. In particular, we have identified non-linear three-body interactions as essential ingredients for the transition to appear. It is thus an example of how complex system-level behaviour can emerge from the interplay between the node dynamics and the topological dynamics in adaptive networks.

Furthermore, the microscopic interactions determining the order of the swarming transition could be identified in the adaptive-network model. From the emergent-level description we have seen that a subcritical pitchfork bifurcation is only obtained if there are also interactions between agents heading in the same direction, in addition to interactions between agents heading in opposite directions. Therefore, a discontinuous transition from a disordered to an ordered state is possible only in this scenario.

We have reproduced the experimental results qualitatively using the non-spatial adaptivenetwork model. This includes also the intermediate regime, in which the insects switch intermittently between the two collective marching directions. Our results suggest that spatial embedding plays a less central role for the understanding of collective motion than previously assumed. Instead, the adaptive nature of the interaction network among the agents (which is a consequence of spatial embedding, of course) appears to be of crucial importance. Nevertheless, our model connects to agent-based spatial models via the survival probability statistics of ordered states in the intermittent regime. At the transition point, the same power-law exponent as in corresponding SPP models is obtained.

Focusing on the adaptive interaction network among the agents allowed us to draw an analogy between swarming and opinion formation processes in social networks, two fields 


\section{Swarming behaviour}

which have been so far considered separately. This is an example of how the "network perspective" can provide an abstract and reduced view on complex systems, thereby revealing similarities between seemingly disparate research fields and phenomena. 


\section{Opinion formation}

In the previous chapter, we have seen how the phenomenon of collective motion can be modelled in terms of a non-spatial adaptive network. This approach was inspired by the similarities of collective motion with the process of opinion formation and the emergence of consensus within groups of interacting individuals. In this chapter, I further discuss the dynamics of opinion formation in social networks and present an adaptive-network model of opinion formation with asymmetric interactions.

In the research on opinion formation in groups of social individuals, one investigates how groups form and organize, how consensus is achieved in society, or what prevents such consensus. Simple, conceptual models of interacting agents are considered for that purpose, in which an agent's opinion is represented by an abstract state variable, and the agents interact with each other in well-defined ways (Conradt and List, 2009. Castellano et al. 2009). The interactions among the agents represent the social influence individuals exert on each other. As a result of the interactions with other agents, for instance, an agent's state (its opinion) can change. If the states of all agents in the population are equal, consensus has been reached.

One of the central models of opinion formation is the paradigmatic voter model (VM) (Holley and Liggett, 1975). In this model, the agents' state can take any value from a discrete set of opinions. The agents' social influence on each other is represented in the following way: an agent that interacts with another one holding a different opinion adopts this opinion with some probability, i.e. "copies" the other agent's state. As the agents adopt each other's opinions, consensus may thus be achieved in the population from an initially inhomogeneous distribution of opinions. The $\mathrm{VM}$ is usually considered on regular lattices or networks, in which the agents interact with their direct neighbours only.

Several other models of opinion formation have been studied, including extensions of the $\mathrm{VM}$ and continuous models (Deffuant et al., 2000), in which the agents' state can take any value from some interval of real numbers (cf. Castellano et al. 2009, and references therein). In this chapter, I focus on the $\mathrm{VM}$ on heterogeneous and adaptive networks. In the $\mathrm{VM}$ on adaptive networks, the agents can not only adopt the opinions of their neighbours but they may also discard their links to dissenting neighbours (which hold a different opinion) and establish new connections to agents holding the same opinion. Instead of reaching global consensus, the network may thus split into disconnected components, within each of which all agents hold the same opinion.

In the following, I discuss the VM on networks and adaptive networks in more detail. Then I present its extension to directed adaptive networks. We will see that the adaptive nature of the network combined with the directionality of the links can give rise to a novel phenomenon that accelerates the network fragmentation and prevents global consensus. 


\subsection{The voter model}

The voter model $(\mathrm{VM})$ is a caricature model of opinion formation in a population of agents, in which each agent's choice of one of two possible opinions is influenced by the opinions of other agents. It was originally introduced as 'a model of spatial conflict' (Clifford and Sudbury, 1973) and later on named "voter model" by Holley and Liggett (1975). In economics and sociology, it is known under the name of "binary choice with externalities" (e.g. Schelling, 1978), and has become one of the most important conceptual models for opinion formation in populations of intelligent agents (Conradt and List, 2009. Castellano et al. 2009).

\subsubsection{Non-adaptive case}

The original version of the $\mathrm{VM}$ consist of $N$ sites on a $d$-dimensional hypercubic lattice, each of which can be in one of two states (opinions) A or B. In each update step, a random site is chosen and assigned the opinion of a random nearest neighbour. Thus, the two homogeneous configurations, in which all sites have the same opinion A or B, are absorbing states. Once the system has reached one of these consensus configurations, no subsequent update step can change the system any more, and it remains in this state.

Due to its similarity with interacting spin systems and its equivalence to certain branching processes (Holley and Liggett, 1975), the VM has attracted considerable attention in the physics literature. There, the main interest lies in the question whether or not an ordered state, i.e. consensus, is achieved in infinite systems, and in which way such an ordered state is approached. In particular, it has been shown that the VM only orders in $d \leq 2$ dimensions. For $d=2$, consensus is achieved by a slow coarsening process that is sharply distinct from the well-known curvature-driven coarsening in, e.g., the Ising model below the critical temperature. It has been argued that the distinct coarsening behaviour of the $\mathrm{VM}$ is found generically at non-equilibrium order-disorder transitions driven by interfacial noise in models containing two dynamically symmetric absorbing states (Dornic et al. 2001). This defines a non-equilibrium universality class, namely the VM universality class (Hinrichsen 2006).

Being a conceptual model for opinion formation among social agents, the VM has also been investigated using more realistic, complex networks as underlying interaction topologies, rather than regular lattices. In the physics literature, the focus is on the effect of different topologies on the ordering dynamics of finite systems. ${ }^{1}$ which has been studied in complex networks (Sood and Redner. 2005 Suchecki et al. 2005a b Castellano, 2005: Ángeles Serrano et al.|2009: Sood et al. |2008: Vazquez and Eguíluz, 2008), including weighted (Baronchelli et al. 2011) and directed networks (Sánchez et al. 2002, jiang et al. 2008). It has been shown that the average time to reach consensus is strongly influenced

${ }^{1}$ The VM does not reach consensus in complex networks of infinite size, as they generally correspond to $d \rightarrow \infty$ (Albert and Barabási. 2002. Dorogovtsev et al. 2008). What is of interest here is how finite networks approach consensus, which is always achieved due to finite size fluctuations. 

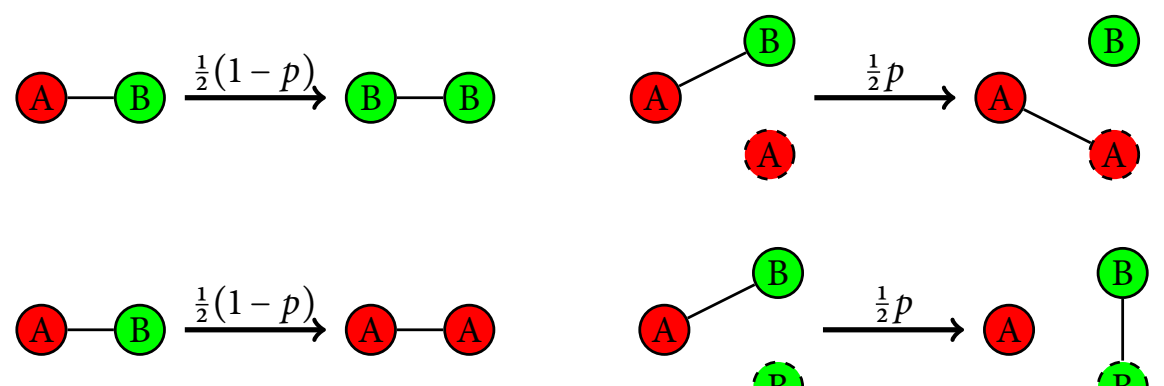

Figure 5.1.: Transformation rules for the adaptive VM Dashed circles denote A- or B-nodes picked at random from the whole population.

by the degree distribution in finite heterogeneous networks (Sood and Redner. 2005. Suchecki et al. 2005b).

\subsubsection{Adaptive voter model}

In order to improve the realism of the $\mathrm{VM}$ as a metaphor for opinion formation in social networks, Holme and Newman (2006) considered an extension of the model, in which they introduced homophily among the agents. Homophily is the tendency of social individuals to prefer interactions with like-minded or otherwise similar others (McPherson et al. 2001). If the individuals in a population are free to choose with whom to interact, homophily leads to the segregation of different groups in the population (Schelling, 1969).

In the model of Holme and Newman, the agents adopt the opinion of random neighbours as in the original $\mathrm{VM}$. In addition to that, the agents can discard links to neighbours of different opinion and rewire them to random agents holding the same opinion as themselves. Thus, the interaction network does not remain fixed but evolves in time. Moreover, the interaction network is an adaptive network because the topological evolution is tightly coupled to the local opinion dynamics.

The adaptive VM is generally studied under the direct node update scheme, in which a random agent is selected in each update step to adopt the opinion of a randomly selected neighbour or rewire the link between them (Holme and Newman, 2006, Vazquez et al. 2008). In the following, however, I consider this model under the link update scheme, in which a random active link is selected in the first place. We will see that this does not change the model behaviour qualitatively but allows for a somewhat simpler analysis. Moreover, it facilitates the comparison with the subsequent extension of the model to directed networks.

The adaptive $\mathrm{VM}$ evolves according to the transformation rules in Fig. 5.1, which are applied using the link update scheme. Every node $i$ holds an opinion $\sigma_{i} \in \mathcal{S}$. In each update step, a random link $(i, j)$ is selected from the network. If $\sigma_{i}=\sigma_{j}$, the link is said 


\section{Opinion formation}

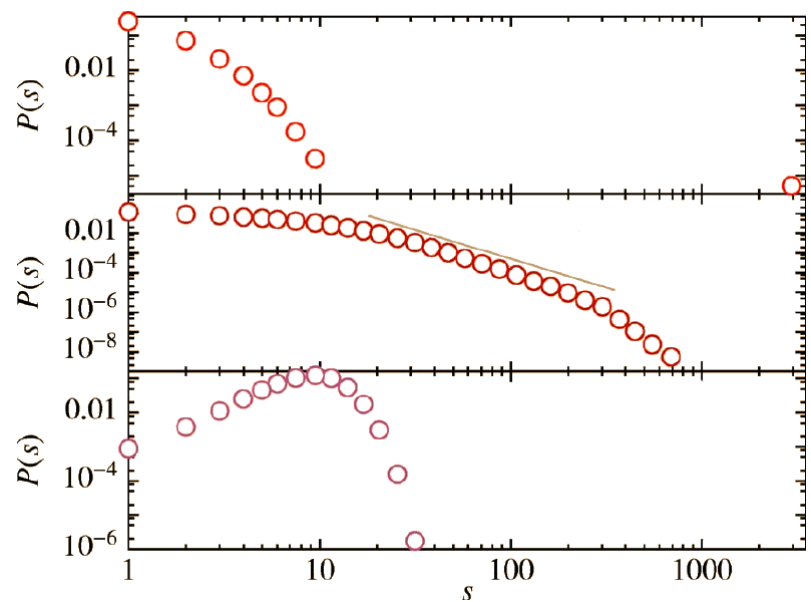

Figure 5.2.: Size distribution of consensus components in the adaptive VM with $g$ opinions for rewiring rates below ( $p=0.04$, top), at $(p=0.458$, center), and above the critical point ( $p=0.96$, bottom). Circles denote averages over $10^{4}$ simulation runs using the direct node update scheme. Parameters: $N=3200,\langle k\rangle=4, g=320$. Figure taken from (Holme and Newman. 2006).

to be inert, and nothing happens. If $\sigma_{i} \neq \sigma_{j}$, the link is said to be active. In this case, it is either rewired, or one of the nodes adopts the opinion of its neighbour. More precisely, the link $(i, j)$ is rewired to become a link $(i, k)$ with probability $p / 2$ or to become a link $(l, j)$ with the same probability $p / 2$, where the node $k(l)$ is randomly selected from all nodes holding the same opinion as $i(j)$. Otherwise, with probability $(1-p) / 2$, node $i$ updates its opinion $\sigma_{i}$ to $j$ 's opinion $\sigma_{j}$, or node $j$ updates its opinion $\sigma_{j}$ to $i$ 's opinion $\sigma_{i}$ with the same probability. The rewiring rate $p$ thus parametrizes the relative time scales of social adjustment (adopting the opinion of a neighbour) and segregation (rewiring). For small $p$, the nodes tend to revise their opinions, while they tend to change their interaction partners for $p \rightarrow 1$. Holme and Newman (2006) investigated numerically the general case where each agent could initially adopt one of $a b s \mathcal{S}=g$ possible opinions, similar to a $g$-state Potts model. Subsequently, Vazquez et al. (2008) focused on the analytical treatment of the case $g=2$, which corresponds to the situation in the original non-adaptive VM

Note that all transformation rules require the presence of an active link, i.e. an A-B-link, on the LHS. If the network reaches a configuration in which there remain no active links, the dynamics stops. Hence, the states with zero active links are absorbing states of the system. In these states, all nodes in the same connected component hold the same opinion. The size distribution of these consensus components depends crucially on the rewiring rate $p$, as is apparent in the simulation results shown in Fig. 5.2 If $p=0$, no links are rewired and the consensus components correspond to the connected components of the initial ER network, i.e. one giant component whose size is $O(N)$ and $O(N)$ small components whose size is $O(1)$ (Albert and Barabási 2002). If $p=1$, on the other hand, 
the nodes do not change their opinions and only rewire their links. In this case, the network splits into many small components whose size distribution equals the initial distribution of opinions. Between these two limits, a continuous phase transition takes place at a critical rewiring rate $p_{c}$, at which the component size distribution follows a power law. As this transition occurs between a global consensus phase, in which most of the network holds the same opinion, and a fragmented phase, in which the network is split into different consensus components, it is called the fragmentation transition. It has also been observed in other variants of the adaptive VM (Gil and Zanette, 2006, Nardini et al. 2008. Kimura and Hayakawa, 2008).

If there are only two possible opinions $(\mathcal{S}=\{\mathrm{A}, \mathrm{B}\})$ initially distributed homogeneously with equal probabilities, the network splits into two disconnected components of approximately the same size for $p>p_{c}$. In this case, the dynamics below and above the transition can be further understood from the approximate analytical description of the system in terms of moment equations (Vazquez et al. 2008, Kimura and Hayakawa. 2008).

To prepare the discussion of the VM in directed adaptive networks, I briefly present the moment equations for the undirected case. We consider the basic network moments [A], the density of nodes in state $\mathrm{A}$, and $[\mathrm{AB}]$, the per-capita density of A-B-links. Following 3.3. we obtain a set of coupled ODE for these moments. The change in the density of A-nodes is given by the sum over all opinion adoption events as

$$
\frac{\mathrm{d}}{\mathrm{dt}}[\mathrm{A}]=\sum_{\sigma_{i j}=\mathrm{AB}} \frac{1-p}{2} N^{-1}-\sum_{\sigma_{i j}=\mathrm{AB}} \frac{1-p}{2} N^{-1}=0 .
$$

Here, the summation runs over all active links because the node state changes are link events under the link update scheme. The first term represents the gain in A-nodes when the B-node in an A-B-link adopts the opinion of its A-neighbour, and the second term represents the loss when the A-node adopts the opinion of its B-neighbour. These events change [A] by $\pm N^{-1}$. As the transformation rules are symmetric with respect to the two possible opinions, i.e. they are invariant under the exchange $\mathrm{A} \leftrightarrow \mathrm{B}$, the two contributions cancel. Hence, any initial density of A-nodes is conserved in infinite systems. This is a direct consequence of using the link update scheme, whereas the same is only true under the direct node update scheme if one additionally assumes the average degrees of A- and B-nodes to be equal (Vazquez et al. 2008).

The density of active links, $[\mathrm{AB}]$, evolves according to

$$
\begin{aligned}
\frac{\mathrm{d}}{\mathrm{dt}}[\mathrm{AB}]= & -\sum_{\sigma_{i j}=\mathrm{AB}} \frac{p}{2} N^{-1}-\sum_{\sigma_{i j}=\mathrm{AB}} \frac{p}{2} N^{-1} \\
& +\sum_{\sigma_{i j}=\mathrm{AB}} \frac{1-p}{2}\left\{n_{i}(A)-n_{i}(B)\right\} N^{-1} \\
& +\sum_{\sigma_{i j}=\mathrm{AB}} \frac{1-p}{2}\left\{n_{j}(B)-n_{j}(A)\right\} N^{-1},
\end{aligned}
$$




\section{Opinion formation}

where the first line corresponds to rewiring events, which always reduce the number of active links, and the second and third line correspond to opinion adoption events. Here, $n_{i}(\sigma)$ denotes the number of $\sigma$-neighbours of the node $i$. Carrying out the sums in the same way as in section 3.3 , we obtain

$$
\begin{aligned}
\frac{\mathrm{d}}{\mathrm{dt}}[\mathrm{AB}]=-p[\mathrm{AB}] & +\frac{1-p}{2}\{[\mathrm{AAB}]-2[\mathrm{BAB}]-[\mathrm{AB}]\} \\
& +\frac{1-p}{2}\{[\mathrm{BBA}]-2[\mathrm{ABA}]-[\mathrm{AB}]\} .
\end{aligned}
$$

As the adoption of a neighbour's opinion is, in essence, $\mathrm{a} C \mathrm{CP}$ this equation depends again on triplet moments. Therefore, we could either derive further equations for the time evolution of the latter or close the ODE system by means of a suitable approximation. We express the triplet moments in terms of link and node densities using the $\mathrm{PA}$ according to Eq. 3.13 and obtain

$$
\begin{aligned}
\frac{\mathrm{d}}{\mathrm{dt}}[\mathrm{AB}]=-p[\mathrm{AB}]+(1-p)[\mathrm{AB}] & +\frac{1-p}{2}\left\{\kappa_{\mathrm{A}} \frac{2[\mathrm{AA}][\mathrm{AB}]}{[\mathrm{A}]}-\kappa_{\mathrm{A}} \frac{[\mathrm{AB}]^{2}}{[\mathrm{~A}]}\right\} \\
+ & \frac{1-p}{2}\left\{\kappa_{\mathrm{B}} \frac{2[\mathrm{BB}][\mathrm{AB}]}{[\mathrm{B}]}-\kappa_{\mathrm{B}} \frac{[\mathrm{AB}]^{2}}{[\mathrm{~B}]}\right\},
\end{aligned}
$$

where $\kappa_{\sigma}=\left\langle q_{\sigma}\right\rangle /\left\langle k_{\sigma}\right\rangle$.

For further simplification we assume that the mean degrees and mean excess degrees of the A- and B-nodes are equal, $\left\langle k_{\mathrm{A}}\right\rangle=\left\langle k_{\mathrm{B}}\right\rangle=\langle k\rangle$ and $\left\langle q_{\mathrm{A}}\right\rangle=\left\langle q_{\mathrm{B}}\right\rangle=\langle q\rangle$, which is a valid assumption as long as both states are abundant in the system (Vazquez et al. 2008). Thus writing $[\mathrm{AA}]=(\langle k\rangle[\mathrm{A}]-[\mathrm{AB}]) / 2$ and $[\mathrm{BB}]=(\langle k\rangle[\mathrm{B}]-[\mathrm{AB}]) / 2$, we obtain the closed ODE system

$$
\begin{aligned}
\frac{\mathrm{d}}{\mathrm{dt}}[\mathrm{A}] & =0, \\
\frac{\mathrm{d}}{\mathrm{dt}}[\mathrm{AB}] & =[\mathrm{AB}]\left\{(1-p) \kappa\left(\langle k\rangle-\frac{[\mathrm{AB}]}{[\mathrm{A}](1-[\mathrm{A}])}\right)-1\right\},
\end{aligned}
$$

with $\kappa=\langle q\rangle /\langle k\rangle$. Any initial density of A-nodes is conserved while the density of active links evolves according to (5.6). The node states A and B are, in fact, arbitrary labels and completely interchangeable. Therefore, meaningful variables are the magnetization $m=[\mathrm{A}]-[\mathrm{B}]=1-2[\mathrm{~A}]$ and the (conveniently normalized) active link density $\rho=$ $2[\mathrm{AB}] /\langle k\rangle$.

The ODE system has two stationary states, which we write in terms of $m$ and $\rho$ as

$$
\begin{array}{ll}
\rho_{1}^{*}=0, & m_{1}^{*} \in[-1,1], \\
\rho_{2}^{*}=\frac{1-\left(m_{2}^{*}\right)^{2}}{2}\left\{1-\frac{1}{(1-p) \kappa\langle k\rangle}\right\}, & m_{2}^{*} \in[-1,1] .
\end{array}
$$


In both cases, $m^{*}$ is arbitrary, so that $\rho_{1}^{*}$ and $\rho_{2}^{*}$ actually describe two families of stationary states in the $m-\rho$-plane. The first family corresponds to the absorbing states in the system, in which there are no active links $\left(\rho_{1}^{*}=0\right)$. In these states, either global consensus is achieved $(m= \pm 1)$, or the network is fragmented into two disconnected components $(-1<m<1)$. The second family corresponds to active states, i.e. dynamical equilibria in which a constant fraction of links is active.

It is instructive to compare equations (5.7) 5.8 with the analogous results obtained for the original adaptive $\mathrm{VM}$ under the direct node update scheme (Vazquez et al., 2008),

$$
\begin{array}{ll}
\rho_{1}^{\mathrm{NU}}=0, & m_{1}^{*} \in[-1,1], \\
\rho_{2}^{\mathrm{NU}}=\frac{1-\left(m_{2}^{*}\right)^{2}}{2}\left\{1-\frac{1}{(1-p)(\langle k\rangle-1)}\right\}, & m_{2}^{*} \in[-1,1] .
\end{array}
$$

The main difference between the link update and the node update results is the appearance of the factor $\kappa$ in the link update equation 5.8. In particular, the factor $(\langle k\rangle-1)$ in 5.10 is replaced by a factor $\kappa\langle k\rangle=\langle q\rangle$ in (5.8), which reflects the influence of the network's degree distribution on the update procedure (cf. 3.1.2).

The factor $\kappa$, however, is unknown because the rewiring dynamics is continually changing the degree distribution. Although the mean degree $\langle k\rangle$ is not affected by the rewiring of links (the total number of links is fixed), the second moment of the degree distribution, and hence $\langle q\rangle$, does not remain constant. Therefore, $\kappa$ must be treated as an additional parameter that can be fitted to simulation data or approximated using suitable assumptions. As the rewiring dynamics creates and destroys links largely at random (active links are updated randomly and links are rewired to random nodes), we expect the emerging degree distribution to remain relatively close to the Poisson distribution of the initial ER network. Therefore, a reasonable and practical approximation is $\kappa=1$, which is exact for a Poissonian degree distribution (cf.2.1.).

The stability of the two families of stationary states depends on the rewiring rate $p$. At small $p$, the active states $\rho_{2}^{*}$ are stable, whereas the absorbing fragmented states $\rho_{1}^{*}$ are stable for large $p$ because fast rewiring can efficiently separate the network into two components. The linear stability analysis of the system reveals a transcritical bifurcation ${ }^{2}$ at

$$
p_{c}=1-\frac{1}{\kappa\langle k\rangle}=1-\frac{1}{\langle q\rangle}
$$

at which the active and absorbing states exchange stability (Fig. 5.3). Again, this can be compared to the analogous result for the direct node update scheme,

$$
p_{c}^{\mathrm{NU}}=1-\frac{1}{\langle k\rangle-1}
$$

where the term $\langle k\rangle-1$ appears again instead of $\langle q\rangle$.

\footnotetext{
${ }^{2}$ See appendix $\sqrt{B}$ for an overview of the bifurcation types that play a role in the present work.
} 


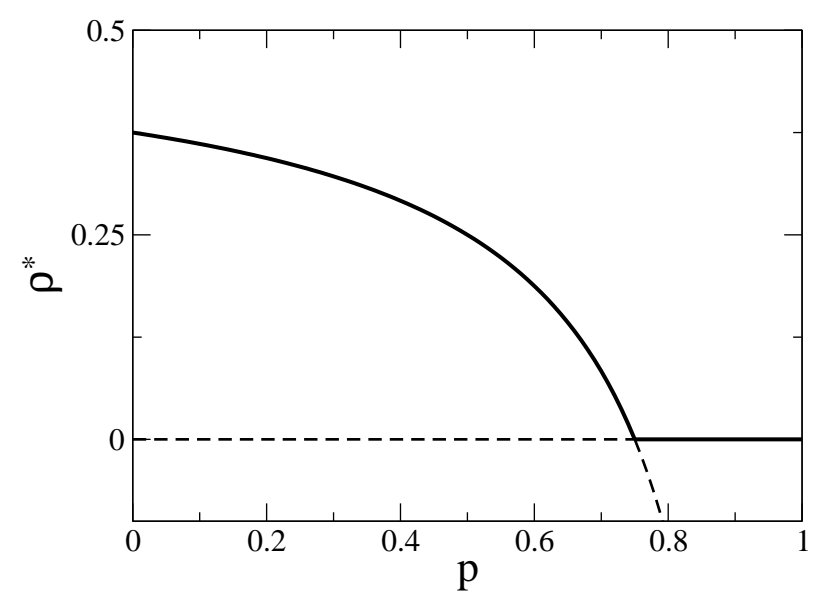

Figure 5.3.: Bifurcation diagram of the adaptive $\operatorname{VM}$ for $\langle k\rangle=4, m^{*}=0$, and $\kappa=1$. The active state $\rho^{*}>0$ and the absorbing state $\rho^{*}=0$ exchange stability in a transcritical bifurcation at $p_{c}=3 / 4$. The unstable stationary state $\rho^{*}<0$ is a non-physical solution of the moment equations.

The parabola of active stationary states becomes unstable at the critical rewiring rate $p_{c}$, where it coincides with the line of absorbing states with $\rho=0$. Moreover, for $p>p_{c}$ the now unstable active states predicted by (5.8) are non-physical solutions of the ODE with $\rho<0$ and hence cannot be accessed by the system.

In simulations of the adaptive $\mathrm{VM}$, the system always reaches one of the absorbing states due to the finite network size. Below the fragmentation transition the typical trajectories in the $m-\rho$-plane follow a characteristic parabola corresponding to the stable stationary states in the infinite system, as shown in Fig. 5.4 Starting from an ER network with randomly assigned, equiprobable node states, the system quickly approaches the parabola and then randomly drifts along this line of stationary states until it reaches one of the absorbing consensus states with $m= \pm 1$. Nevertheless, the predicted parabola $\rho_{2}^{*}\left(m^{*}\right)$ according to $(5.8)$ consistently overestimates the observed active link density if the Poisson approximation $\kappa=1$ is used. A better agreement with the simulation results is obtained for lower values of $\kappa$, indicating that the rewiring dynamics tends to generate a narrower degree distribution than the original Poisson distribution. This is indeed observed in simulations. However, although a parameter $\kappa<1$ is measured, its deviation from the Poisson approximation is in general much smaller than what would be needed to fit Eq. 5.8 to the experimental data. Therefore, the mismatch between the $\mathrm{PA}$ result and the simulation data must be additionally attributed to emerging correlations not taken into account in the approximations (Demirel et al. 2011b).

At large rewiring rates, the typical trajectories quickly reach a fragmented absorbing state, in which the network has split into two disconnected components of roughly the same size. As shown in Fig. 5.5, the fragmentation transition is clearly visible in the absolute value of the magnetization $m$ in the final state in simulations. However, the 
5.1. The voter model

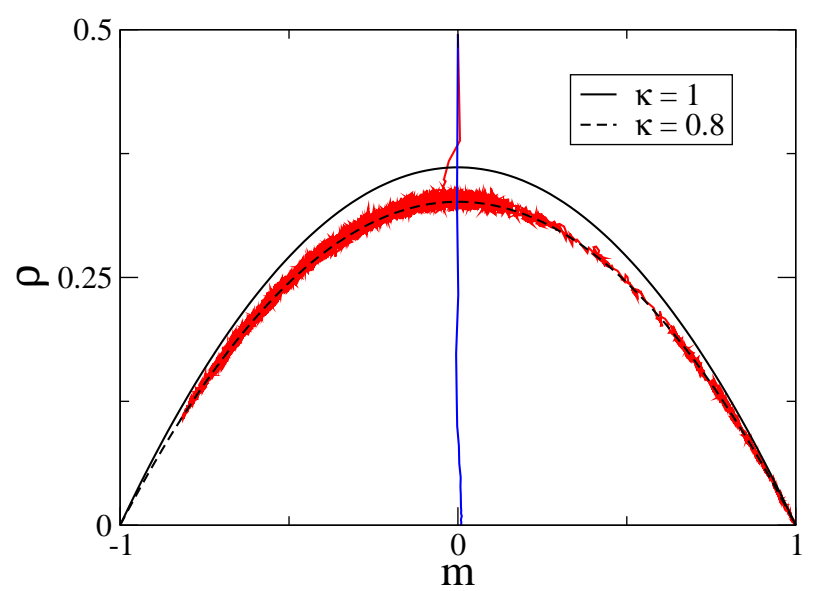

Figure 5.4.: Typical trajectories of the adaptive $\mathrm{VM}$ in the $m$ - $\rho$-plane. The network is initialized as an ER network with equiprobable node states $(m=0, \rho=0.5)$. For $p<p_{c}$ (red), the trajectory drifts along the parabola of active stationary states until a consensus state $(m= \pm 1, \rho=0)$ is reached. For $p>p_{c}$ (blue), the trajectory quickly reaches a fragmented absorbing state. Note that the Poisson approximation for the unknown parameter $\kappa, \kappa=1$, consistently overestimates the density of active links. Parameters: $N=10^{4},\langle k\rangle=4, p=0.1$ (red), $p=0.8$ (blue).

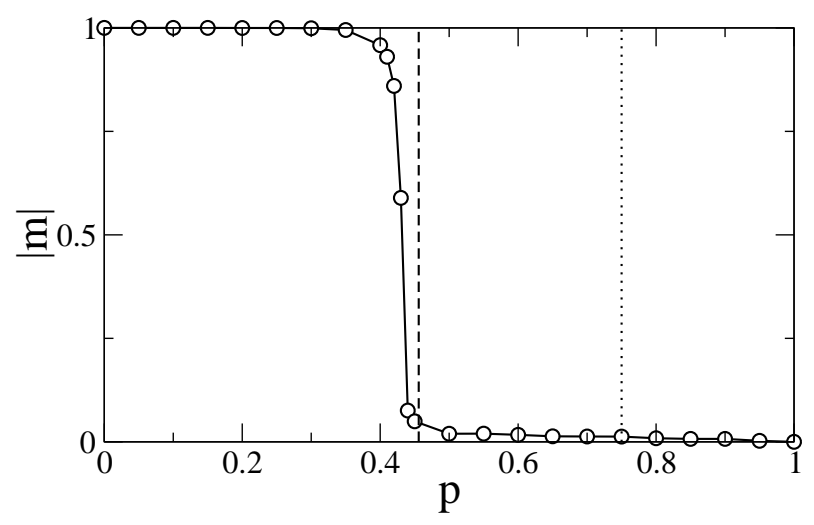

Figure 5.5.: Fragmentation transition in simulations of the adaptive VM Shown is the absolute value of the magnetization $m$ in the final state for different rewiring rates $p$. The critical point computed from the moment equations (dotted, for $\kappa=1$ ) overestimates the critical rewiring rate, whereas the motif expansion yields a better estimate of the fragmentation point (dashed). Parameters: $N=10^{4},\langle k\rangle=4$. 


\section{Opinion formation}

critical rewiring rate (5.11) overestimates the transition point significantly.

Although the moment equations fail to predict the model behaviour quantitatively, they provide a qualitative understanding of the voter dynamics. The characteristic parabolic shape of the system trajectories in the $m$ - $\rho$-plane is readily explained by the family of stable, active stationary states in 5.8 . Moreover, the fragmentation transition can be understood from the destabilization of these states at a critical rewiring rate $p_{c}$. For $p>p_{c}$, the active states are inaccessible to the system (and unstable). Therefore, the system trajectories are attracted by the now stable absorbing states. As the magnetization is conserved, fragmentation is observed for any initial magnetization with $|m| \neq 1$.

A more accurate estimate for the critical rewiring rate can be obtained using a different analytical approach that was proposed by Böhme and Gross (2011). Instead of tracking the time evolution of simple network moments starting from a random, connected network, the dynamics of the last remaining connections shortly before fragmentation is analysed in this approach. To that end, an almost fragmented network is considered, consisting of two almost isolated components which are internally in consensus, but are still connected by a low density of "active motifs". An active motif is a star-like subgraph containing at least one active link.

In the so-called motif expansion, a series of balance equations is derived for the time evolution of the densities of active motifs. Fragmentation is achieved if the active motifs cannot proliferate and invade the two consensus components but die out instead. From this condition, the critical fragmentation point can be obtained. This is done by analysing the linear stability of the absorbing stationary state that contains zero active motifs. The critical rewiring rate $p_{c}$ is the value of $p$ at which this state becomes a stable stationary state of the motif expansion equations. Using this approach, the fragmentation point observed in simulations is well approximated (Fig. 5.5).

The adaptive VM has been studied so far on undirected networks, assuming the social interactions through which the agents adjust their opinions to be symmetric. In the context of opinion formation in social groups, however, the interaction network represents "who can influence whom" or "who listens to whom", which are inherently asymmetric inter-agent relations. It is therefore reasonable to study opinion formation and voter dynamics on directed networks, encoding "who pays attention to whom" as directed links among the agents.

While opinion formation on static directed networks has been investigated in various models, including several variants of the non-adaptive VM (Sánchez et al. 2002. Park and Kim, 2006; Jiang et al., 2008. Ángeles Serrano et al. 2009. Masuda and Ohtsuki, 2009), little insight has been gained in the dynamics of directed adaptive networks. A generic threshold model for directed Boolean networks that bears some similarity with the adaptive VM has been investigated by Lambiotte and González-Avella (2011). They observe a similar fragmentation transition as in the adaptive VM in their model, indicating that the characteristic dynamics in undirected networks may carry over to the directed case. To the author's knowledge, the original adaptive VM has not yet been studied on 
directed networks.

Directed links representing the possible directions of opinion transfer introduce an asymmetry in the local update rules, as they assign distinct roles to the nodes they connect. Which of the two nodes adopts the opinion of the other in a directed active link, for instance, is determined by the link direction, whereas either node can copy from the other in an undirected link. This symmetry breaking can be expected to give rise to novel phenomena in directed adaptive networks, which are not observed in their undirected counterparts. In the next section, I present an extension of the adaptive VM to directed networks, in which the interplay between the link directionality and the adaptive rewiring accelerates the network fragmentation in comparison with the undirected case. We will see that this is achieved by a novel mechanism that is enabled by the asymmetry of the interaction network.

\subsection{Early fragmentation in directed adaptive networks $3^{3}$}

In this section, we investigate voter dynamics in a directed adaptive network, in which both the opinion dynamics and the topological change are influenced by the directionality of the interactions. In our model, the agents' relationships are represented by directed links, which encode "who listens to whom" in the system. An agent may adopt the opinion of a neighbour it pays attention to, i.e. to which it has an outgoing link. The agents can avoid disagreeing with neighbours by rewiring their outgoing links, whereas they cannot affect their incoming links (which represent the influence they have on other agents). The overall network topology thus changes adaptively, while the out-degree of each agent, i.e. its number of outgoing links remains unchanged. This enables us to study the influence of different realistic out-degree distributions. We find that for sufficiently dense Poissonian out-degree distributions, fragmentation occurs if the rewiring of network connections exceeds a critical rate, which is consistent with previous results on undirected networks. However, for scale-free out-degree distributions and Poissonian distributions with small mean degree, fragmentation can already be observed at much lower rewiring rates than in undirected networks. We show that this behaviour is due to the nodes of low out-degree, which can form self-stabilizing topological structures that nucleate fragmentation.

\subsubsection{Model}

We consider a network of $N$ nodes representing agents and $K$ directed links representing social interactions, so that the average in- and out-degree of the nodes are $\langle k\rangle_{\mathrm{i}}=\langle k\rangle_{\mathrm{o}}=$ $\langle k\rangle=K / N]^{\mid}$Each node $i$ holds a binary opinion $\sigma_{i} \in\{\mathrm{A}, \mathrm{B}\}$. The direction of links

${ }^{3}$ This section is based on the author's article (Zschaler et al. 2012) published in Physical Review E.

${ }^{4}$ Note that we write $\langle k\rangle=K / N$ for the average in-degree and the average out-degree of the nodes here, which must necessarily be equal in any well-formed directed network. In an undirected network, the average degree is $\langle k\rangle=2 \mathrm{~K} / \mathrm{N}$ because every link contributes to the average degree of both nodes it connects. 

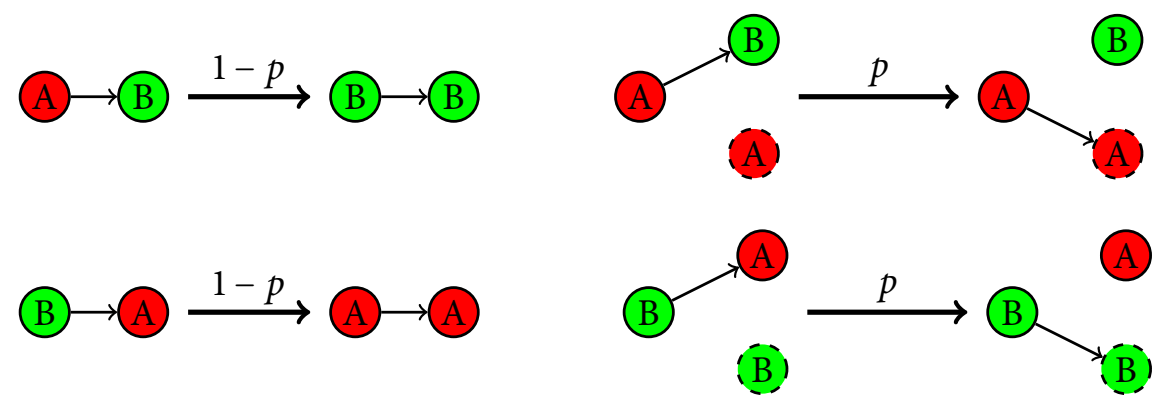

Figure 5.6.: Transformation rules for the directed adaptive VM Dashed circles denote A- or $\mathrm{B}$-nodes picked at random from the whole population.

indicates the flow of attention between the agents. In other words, in our notation we draw links in the direction that one would draw the "follows"-links in the micro-blogging service Twitter, for instance.

We initialize the network as a random directed graph ${ }^{5}$ with randomly assigned equiprobable strategies. The node states and the network topology are then left to evolve according to the transformation rules shown in Fig. 5.6 These rules are analogous to the transformation rules in the undirected $\mathrm{VM}$ (cf. 5.1.2), except that an additional asymmetry is introduced by the link directionality. Using the link update scheme, a link $i \rightarrow j$ is picked at random from the network. If it is an inert link $\left(\sigma_{i}=\sigma_{j}\right)$, nothing happens. Otherwise, the active link is either rewired (probability $p$ ), or an opinion update takes place (probability $1-p$ ). In the former case, the node $i$ cuts the link and reconnects to a random node $k$ with $\sigma_{k}=\sigma_{i}$. In the latter case, node $i$ switches its opinion $\sigma_{i}$ to $\sigma_{j}$. In contrast to the undirected $\mathrm{VM}$, it is always the node $i$ in an $i \rightarrow j$-link that changes its opinion or rewires the link. Therefore, the rewiring of links only changes the in-degree distribution, whereas the out-degree distribution and the average degree $\langle k\rangle=K / N$ of the network remain fixed.

In contrast to previous studies of the VM on static directed networks, we do not need to restrict our model to networks consisting of a single strongly connected component ${ }^{6}$ (Dorogovtsev et al. 2001: Sánchez et al. 2002, Ángeles Serrano et al., 2009), because the network's component structure is affected by the ongoing rewiring of links, which continuously forms and re-routes paths between different strongly connected components.

Below, we study the proposed model in terms of the density $n$ of A-nodes (corresponding to agents holding opinion A) and the per-capita densities $f$ and $h$ of active links, $f \equiv[\mathrm{A} \rightarrow \mathrm{B}]$ and $h \equiv[\mathrm{B} \rightarrow \mathrm{A}]$. To be able to compare our results with the undirected model, we characterize the state of the network by the magnetization $m=1-2 n$ and the active link density $\rho=(f+h) /\langle k\rangle$.

\footnotetext{
${ }^{5} \mathrm{~A}$ random directed graph is constructed from an undirected ER graph by assigning to each link a random direction.

${ }^{6} \mathrm{~A}$ strongly connected component of a directed network is a maximal subgraph in which there is a directed path from each node to all other nodes in this subgraph (Newman 2010).
} 


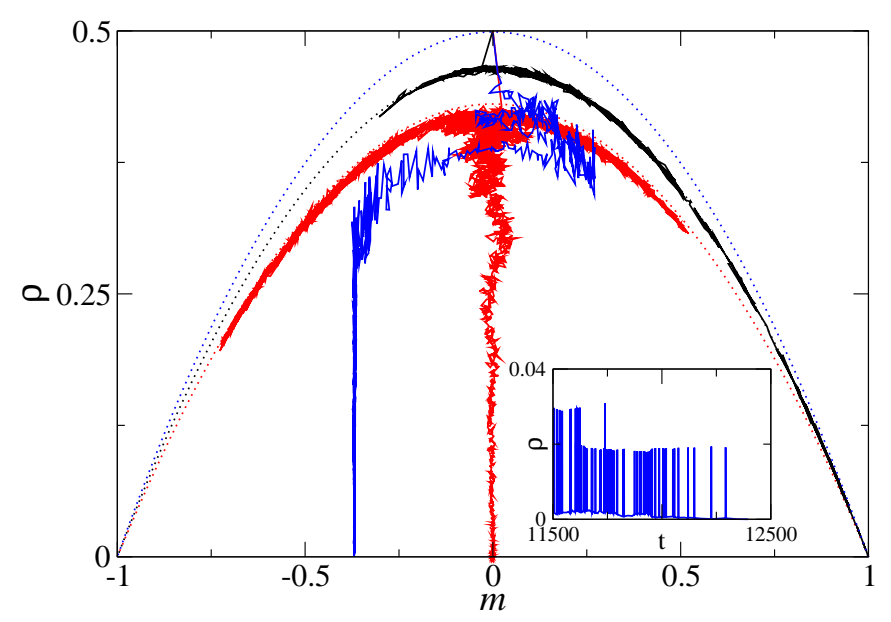

Figure 5.7.: Typical trajectories of the directed adaptive VM in the $m$ - $\rho$-plane below the estimated fragmentation point. Shown are trajectories corresponding to networks with Poissonian outdegree distributions with $\langle k\rangle=4$ (red), $\langle k\rangle=8$ (black), and an out-degree distribution following $p_{k} \propto k^{-2}$ (blue). The trajectories initially drift along a parabola of active states (dotted lines in matching colours, analytical results from Eq. (5.22)). However, only the black trajectory reaches a consensus state, whereas the others eventually collapse to a fragmented state. The inset shows a time series of $\rho$ from the scale-free network shortly before fragmentation. $N=10^{4}, p=0.1$.

In network simulations of the directed adaptive $\mathrm{VM}$, one observes qualitatively different types of trajectories: First at sufficiently high rewiring rates, the network rapidly approaches a fragmented state $(|m|>0, \rho=0)$, in which the network breaks into at least two components, which are internally in consensus. Second, for sufficiently low rewiring rates the network first approaches a stationary active state $(|m| \ll 1, \rho>0)$, in which the opinions and the topology change continually. Because such active states form a parabola in the $m$ - $\rho$-plane, the system can drift randomly along the parabola until an absorbing consensus state $(m= \pm 1, \rho=0)$ is reached. These dynamics are closely reminiscent of the dynamics observed in the undirected adaptive VM discussed in 5.1.2 and can be explained by an analogous analytical approach, as we will see in the next section.

In addition to the trajectories described above, the directed model can show a third type of behaviour not observed in the undirected case. Here, the systems drifts along the parabola of active states for some time and then collapses slowly to the fragmented state (Fig. 5.7). This can lead to fragmentation significantly below the critical rewiring rate found in undirected networks. The delayed fragmentation after the drift along the parabola of active states suggests that in the active state the network undergoes some slow reorganization that eventually destabilizes the active states. In the following, we investigate the nature of this reorganization and its implications for the network fragmentation. 


\section{Opinion formation}

\subsubsection{Analytical approach}

To explore the impact the directionality of attention has on the opinion formation process, we compare the dynamics of the directed adaptive $\mathrm{VM}$ to the undirected adaptive VM In the following we refer to these two models simply as the directed model and the undirected model, respectively. We compare simulation results of the directed model with the analytical approximations that are known to capture the dynamics of the undirected model in different limits, as discussed in 5.1.2 In this comparison an agreement between analytical and numerical results indicates that the assumptions made for the undirected model are still valid in the directed model, whereas a disagreement points to new physics in the directed model that is not observed in the undirected model.

\section{Moment expansion}

Following the discussion in 5.1.2 we derive differential equations for the time evolution of the network moments, namely the densities $n, f$, and $h$ defined above. The change in the density of A-nodes, $n$, is given by the balance between opinion adoption in $\mathrm{B} \rightarrow \mathrm{A}$ - and $\mathrm{A} \rightarrow \mathrm{B}$-links,

$$
\frac{\mathrm{d}}{\mathrm{dt}} n=(1-p)(h-f),
$$

which does not vanish as in the undirected case due to the asymmetry between the two different active link types.

The density of $\mathrm{A} \rightarrow \mathrm{B}$-links, $f$, changes according to

$$
\frac{\mathrm{d}}{\mathrm{dt}} f=-p f+(1-p)\{[\mathrm{A} \rightarrow \mathrm{A} \rightarrow \mathrm{B}]-2[\mathrm{~B} \leftarrow \mathrm{A} \rightarrow \mathrm{B}]+[\mathrm{B} \leftarrow \mathrm{B} \rightarrow \mathrm{A}]-[\mathrm{A} \rightarrow \mathrm{B} \rightarrow \mathrm{A}]-f\},
$$

where the two types of per-capita triplet densities $[X \rightarrow Y \rightarrow Z]$ and $[X \leftarrow Y \rightarrow Z]$ enter the equations $(X, Y, Z \in\{\mathrm{A}, \mathrm{B}\})$. Here, the first term corresponds to the gain in $f$ due to rewiring, whereas the remaining terms correspond to gains and losses due to opinion adoption. In an opinion adoption event, a node copies a neighbour's opinion via one of its outgoing active links, transforming it into an inert link. As in the undirected model, this also affects all other links connected to the focal node so that active links are transformed into inert ones and vice versa. The resulting indirect change in the density of active links is accounted for by the triplet variables.

The time evolution of the density of $\mathrm{B} \rightarrow \mathrm{A}$-links, $h$, is determined by the analogous equation

$$
\frac{\mathrm{d}}{\mathrm{dt}} h=-p h+(1-p)\{[\mathrm{B} \rightarrow \mathrm{B} \rightarrow \mathrm{A}]-2[\mathrm{~A} \leftarrow \mathrm{B} \rightarrow \mathrm{A}]+[\mathrm{A} \leftarrow \mathrm{A} \rightarrow \mathrm{B}]-[\mathrm{B} \rightarrow \mathrm{A} \rightarrow \mathrm{B}]-h\} .
$$

Equations (5.13)-5.15) do not constitute a closed ODE system, as they involve the triplet moments $[X \rightarrow Y \rightarrow Z]$ and $[X \leftarrow Y \rightarrow Z]$. In principle, the equation system could be complemented by similar equations for the triplet moments. These would, however, 
depend on higher moments, such as four-node motifs. In order to obtain a closed system of equations, we use the PA to approximate the triplet densities in terms of node and link densities.

Following the argument in section 3.3.2, we express the triplet densities as

$$
\begin{array}{r}
{[X \rightarrow Y \rightarrow Z] \approx[X \rightarrow Y]\left\langle k_{Y}\right\rangle \frac{[Y \rightarrow Z]}{\left\langle k_{Y}\right\rangle[Y]},} \\
\mu_{X Z}[X \leftarrow Y \rightarrow Z] \approx[Y \rightarrow X]\left\langle q_{Y}\right\rangle_{\mathrm{o}} \frac{[Y \rightarrow Z]}{\left\langle k_{Y}\right\rangle[Y]},
\end{array}
$$

where $\mu_{X Z}=1+\delta_{X Z}$ accounts for the double-counting of symmetric triplets. These equations are the equivalent of Eq. (3.13) for directed networks. We approximate the numbers of triplets as the number of $X \rightarrow Y$ - or $Y \rightarrow X$-links times the average number of $Y \rightarrow Z$-links connected to a $Y$-node, assuming that the probability of finding an outgoing $Z$-neighbour of a $Y$-node is independent of the presence of an $X$-neighbour of the $Y$ node. Thus the probability of finding a given triplet depends on the global link density $[Y \rightarrow Z] /\left\langle k_{Y}\right\rangle[Y]$. In (5.16), each of the $\left\langle k_{Y}\right\rangle$ outgoing links of the $Y$-node is a $Y \rightarrow Z$-link with this probability. In (5.17), on the other hand, the $Y$-node has already been selected by following one of its outgoing links. In this case, each of its remaining $\left\langle q_{Y}\right\rangle_{\mathrm{O}}$ outgoing links is a $Y \rightarrow Z$ link with this probability. The quantity $\left\langle q_{Y}\right\rangle_{0}$ denotes the mean excess out-degree of the $Y$-nodes in the network (cf. 2.1).

As a further simplification we assume again that the average degree and excess degree in the network are independent of the node states, so that $\left\langle k_{\mathrm{A}}\right\rangle=\left\langle k_{\mathrm{B}}\right\rangle=\langle k\rangle$ and $\left\langle q_{\mathrm{A}}\right\rangle_{\mathrm{O}}=\left\langle q_{\mathrm{B}}\right\rangle_{\mathrm{O}}=\langle q\rangle_{\mathrm{o}}$. Simulation results suggest that this is a valid assumption as long as both node states are abundant in the system, similar to the undirected model. With this assumption we can write $[\mathrm{A} \rightarrow \mathrm{A}]=\langle k\rangle n-f$ and $[\mathrm{B} \rightarrow \mathrm{B}]=\langle k\rangle(1-n)-h$ to obtain a closed set of ODE;,

$$
\begin{aligned}
& \frac{\mathrm{d}}{\mathrm{dt}} n=(1-p)(h-f), \\
& \frac{\mathrm{d}}{\mathrm{dt}} f=-p f+(1-p)\left\{\frac{(\langle k\rangle n-f)-\kappa_{\mathrm{o}} f}{n} f+\frac{\kappa_{\mathrm{o}}(\langle k\rangle(1-n)-h)-f}{1-n} h-f\right\}, \\
& \frac{\mathrm{d}}{\mathrm{dt}} h=-p h+(1-p)\left\{\frac{(\langle k\rangle(1-n)-h)-\kappa_{\mathrm{o}} h}{1-n} h+\frac{\kappa_{\mathrm{o}}(\langle k\rangle n-f)-h}{n} f-h\right\},
\end{aligned}
$$

where $\kappa_{\mathrm{o}}=\langle q\rangle_{\mathrm{o}} /\langle k\rangle$.

This system of differential equations has a trivial solution,

$$
h^{*}=f^{*}=0 \text {, }
$$

which corresponds to the absorbing states in which there are no active links. These states can be either fragmented states $\left(0<n^{*}<1\right)$ or consensus states $\left(n^{*}=0\right.$ or $\left.n^{*}=1\right)$. 


\section{Opinion formation}

Additionally, there is a continuum of non-absorbing, active stationary states,

$$
f^{*}=h^{*}=n^{*}\left(1-n^{*}\right)\left(\langle k\rangle-\frac{1}{(1-p)\left(1+\kappa_{\mathrm{o}}\right)}\right),
$$

in which neither consensus nor fragmentation is achieved. These states form the parabola in the $m$ - $\rho$-plane discussed above (Fig. 5.7),

$$
\rho^{*}=\frac{1-\left(m^{*}\right)^{2}}{2}\left\{1-\frac{1}{(1-p)\left(1+\kappa_{\mathrm{o}}\right)\langle k\rangle}\right\} .
$$

This equation closely resembles Eq. (5.8) for the undirected model. The only difference is that the mean degree $\langle k\rangle$ appears with a factor $\kappa_{\mathrm{o}}+1$ instead of $\kappa$. In the limiting case of a Poissonian (out-)degree distribution where $\kappa_{0}=\kappa=1$, the expressions for the undirected and the directed model coincide (taking into account that the 'total' average degree neglecting the link directions is $2\langle k\rangle$ in the directed network).

Comparing the results of the moment expansion for the directed model with the undirected model, we see that the same analytical approach can be used in both cases and predicts qualitatively the same behaviour. In particular, the parabola of active stationary states in the $m-\rho$-plane is again observed in simulations as the low-dimensional manifold along which the trajectories drift randomly for rewiring rates far below fragmentation.

We have seen that the moment expansion becomes less reliable close to the fragmentation point and overestimates $p_{c}$ in the undirected model, which can be predicted with higher accuracy using a motif expansion approach. To compute a better estimate of the fragmentation point in the directed model, we therefore extend this approach to the case of directed networks. It is nevertheless instructive to consider the critical rewiring rate obtained from the ODE system,

$$
\tilde{p}_{c}=1-\frac{1}{\left(\kappa_{\mathrm{o}}+1\right)\langle k\rangle},
$$

which is computed by a linear stability analysis of the active states. For $p<\tilde{p}_{c}$ the active states are stable, whereas the absorbing states are stable for $p>\tilde{p}_{c}$. This estimate of the critical point can be compared with the analogous result (5.11) for the undirected model. The main difference is that in the undirected case, the parameter $\kappa_{\mathrm{o}}$ is unknown and therefore usually set to unity or fitted from simulation data. By contrast, the outgoing degree distribution remains fixed in the directed model, and $\kappa_{\mathrm{o}}$ has to be considered explicitly. In the results presented here, we use values of $\kappa_{\mathrm{o}}$ that are explicitly measured in realizations of the respective out-degree distributions.

\section{Motif expansion}

In the undirected model, a good estimate for the fragmentation point is obtained using the motif expansion approach proposed by Böhme and Gross (2011). This approach can 


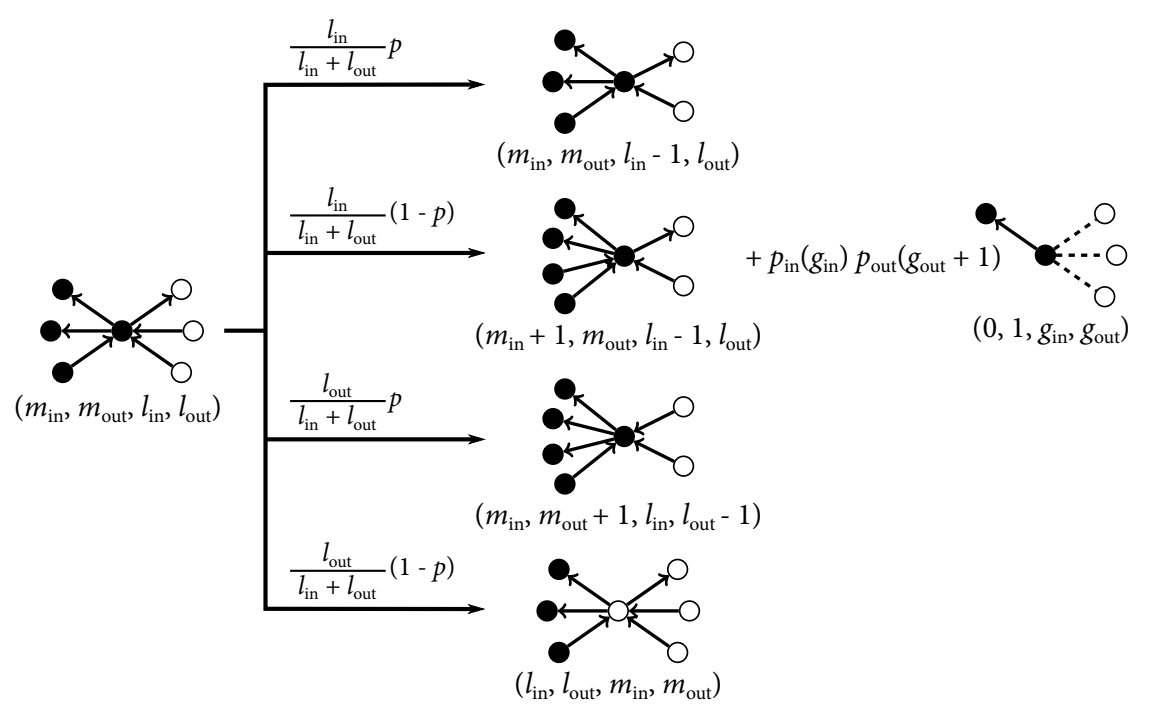

Figure 5.8.: Transitions of a general active motif $\left(m_{\mathrm{in}}, m_{\mathrm{out}}, l_{\mathrm{in}}, l_{\mathrm{out}}\right)$. The transition probabilities are a consequence of the link update rule. New active motifs are only created when an opinion update occurs on an incoming active link (second row). In this case the number of incoming and outgoing active links (dashed) of the new motif is estimated based on the in-degree distribution $p_{\text {in }}$ and out-degree distribution $p_{\text {out }}$ of the network.

be extended straightforwardly to the case of directed networks. In directed networks the active motifs are characterized by their numbers of inert incoming and outgoing links $\left(m_{\mathrm{in}}, m_{\text {out }}\right)$ and active incoming and outgoing links $\left(l_{\text {in }}, l_{\text {out }}\right)$. Following the procedure in (Böhme and Gross, 2011), we derive a set of balance equations capturing the effect of all possible update processes on the densities of active motifs,

$$
\begin{aligned}
\dot{\eta}\left(m_{\text {in }}, m_{\text {out }}, l_{\text {in }}, l_{\text {out }}\right)= & -\eta\left(m_{\text {in }}, m_{\text {out }}, l_{\text {in }}, l_{\text {out }}\right) \\
& +\frac{l_{\text {in }}+1}{l_{\text {in }}+1+l_{\text {out }}} p \eta\left(m_{\text {in }}, m_{\text {out }}, l_{\text {in }}+1, l_{\text {out }}\right) \\
& +\frac{l_{\text {in }}+1}{l_{\text {in }}+1+l_{\text {out }}}(1-p) \eta\left(m_{\text {in }}-1, m_{\text {out }}, l_{\text {in }}+1, l_{\text {out }}\right) \\
& +\frac{l_{\text {out }}+1}{l_{\text {in }}+l_{\text {out }}+1} p \eta\left(m_{\text {in }}, m_{\text {out }}-1, l_{\text {in }}, l_{\text {out }}+1\right) \\
& +\frac{m_{\text {out }}}{m_{\text {in }}+m_{\text {out }}}(1-p) \eta\left(l_{\text {in }}, l_{\text {out }}, m_{\text {in }}, m_{\text {out }}\right)
\end{aligned}
$$

for $m_{\text {in }}>0$ and $m_{\text {out }}>1$, and

$$
\begin{aligned}
\dot{\eta}\left(0,1, l_{\text {in }}, l_{\text {out }}\right) & =-\eta\left(0,1, l_{\text {in }}, l_{\text {out }}\right) \\
& +(1-p) p_{\text {in }}\left(l_{\text {in }}\right) p_{\text {out }}\left(l_{\text {out }}+1\right) \sum \frac{n_{\text {in }}}{n_{\text {in }}+n_{\text {out }}} \eta\left(m_{\text {in }}, m_{\text {out }}, n_{\text {in }}, n_{\text {out }}\right)
\end{aligned}
$$




\section{Opinion formation}

for $m_{\mathrm{in}}=0, m_{\mathrm{out}}=1$. The summation runs over all active motifs $\left(m_{\mathrm{in}}, m_{\mathrm{out}}, n_{\mathrm{in}}, n_{\mathrm{out}}\right)$ up to a maximum in- and out-degree, i.e. over all possible 4-tuples with $m_{\text {in }}+n_{\text {in }} \leq \hat{k}_{\text {in }}$ and $m_{\text {out }}+n_{\text {out }} \leq \hat{k}_{\text {out }}$, where $\hat{k}_{\text {in }}$ and $\hat{k}_{\text {out }}$ denote the cut-offs. Note that the dimension of the transition matrix grows with the cut-off faster than $\hat{k}^{3}$, so that the largest usable cut-offs are strongly limited by the available computation time.

A schematic representation of the transition probabilities is shown in Fig. 5.8 We account for heterogeneous in- and out-degree distributions ( $\left.p_{\text {in }}, p_{\text {out }}\right)$, but assume that the in- and out-degree of a node are uncorrelated. In the balance equations, the fragmented state is obtained as the stationary solution containing zero active motifs. The critical rewiring rate $p_{c}$ is then extracted from the linear stability analysis of this state as the rewiring rate at which the fragmented state becomes stable.

\subsubsection{Numerical exploration of early fragmentation}

In the following, we compare the estimated fragmentation points obtained from the approximations above with results from agent-based simulation of the networks. We first consider the case of a network with Poissonian out-degree distribution with $\langle k\rangle=8$. As a second example we study a network with a scale-free out-degree distribution, in which the fragmentation is observed much earlier. We conjecture that this early fragmentation occurs due to the presence of a large number of nodes with low out-degree, which is then verified in a network with Poissonian degree distribution with $\langle k\rangle=4$.

\section{Poissonian out-degree distribution with $\langle k\rangle=8$}

We first consider networks with a Poissonian out-degree distribution, because this distribution closely matches the distribution observed in the undirected model (Vazquez et al. 2008). Starting from a random graph with both in- and out-degrees drawn from a Poisson distribution with mean $\langle k\rangle$, we simulate the full network dynamics for systems of up to $N=10^{4}$ nodes until a frozen state is reached or a maximum simulation time $t_{\max }$ is exceeded. Time is measured in units of $1 / K$, so that $K$ update events take place in one simulated time unit.

The results in Fig. 5.9 show a relatively sharp fragmentation transition at a critical rewiring rate $p_{c} \approx 0.79$. For $p<p_{c}$, the network reaches a state of global consensus, in which all nodes have the same state $(|m|=1)$. By contrast, for $p>p_{c}$, it separates into two disconnected components of approximately the same size, which hold opposing opinions but are internally in consensus. These results are strongly reminiscent of the undirected model, in which the same fragmentation behaviour is observed (cf. 5.1.2).

The analogy between the directed and undirected model extends also to the analytical results. As in the undirected model, the moment expansion overestimates the transition point, whereas the motif expansion yields a relatively precise estimate.

The study of the directed model in Poissonian networks with $\langle k\rangle=8$ highlights the similarities between the directed and undirected networks and provides a basic test for 


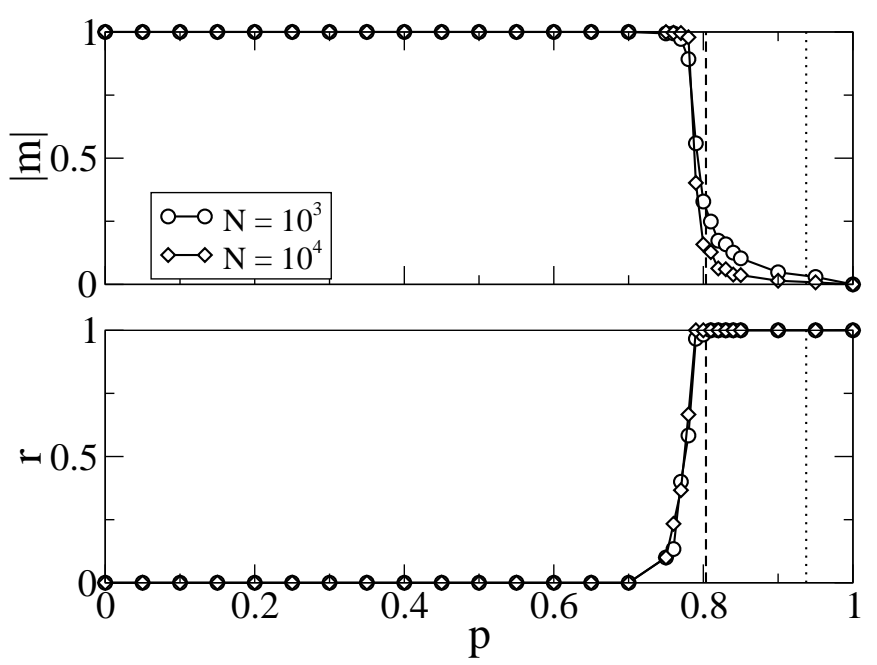

Figure 5.9.: Fragmentation of a network with Poissonian out-degree distribution and $\langle k\rangle=8$. Shown is the absolute value of the magnetization in the final frozen state (top) and the proportion $r$ of simulation runs that reach a fragmented state before $t_{\max }=80000$ (bottom) as a function of the rewiring rate $p$. Each point is an average over 100 simulations. The critical point computed by the moment closure approximation (dotted) overestimates the critical rewiring rate, whereas the motif expansion yields a better estimate of the true fragmentation point. For the motif-expansion a cut-off of $\hat{k}_{\text {in }}=\hat{k}_{\text {out }}=10$ was used. For a higher cut-off the estimated critical rewiring rate is expected to shift to slightly higher values.

our analytical approaches. For these networks, the directed model exhibits the same dynamics as the undirected model, and the analytical approaches capture the dynamics with similar precision as in the undirected case.

\section{Scale-free out-degree distribution}

We now ask how the model behaves for more realistic out-degree distributions which cannot be realized in the previously studied undirected model. In the following, we consider power-law distributions of the form $p_{k} \propto k^{-2}$, which capture the diversity that is observed in a wide variety of social applications (Barabási and Albert, 1999). To generate networks with power-law distributed out-degrees and Poissonian in-degrees, we first draw an out-degree sequence of length $N$ from a power-law distribution. For each out-degree $k_{i}$ in this sequence, we then connect the outgoing links of node $i$ to $k_{i}$ random nodes in the network. We explicitly avoid creating nodes without outgoing links as these would never change their state and act as "zealots", trivially preventing the possibility of global consensus (Mobilia et al., 2007).

The results in Fig. 5.10 show that in scale-free networks the fragmentation occurs much earlier, that is at lower rewiring rates than in the Poissonian case. Moreover, the proportion $r$ of networks reaching fragmentation now increases gradually with increasing 


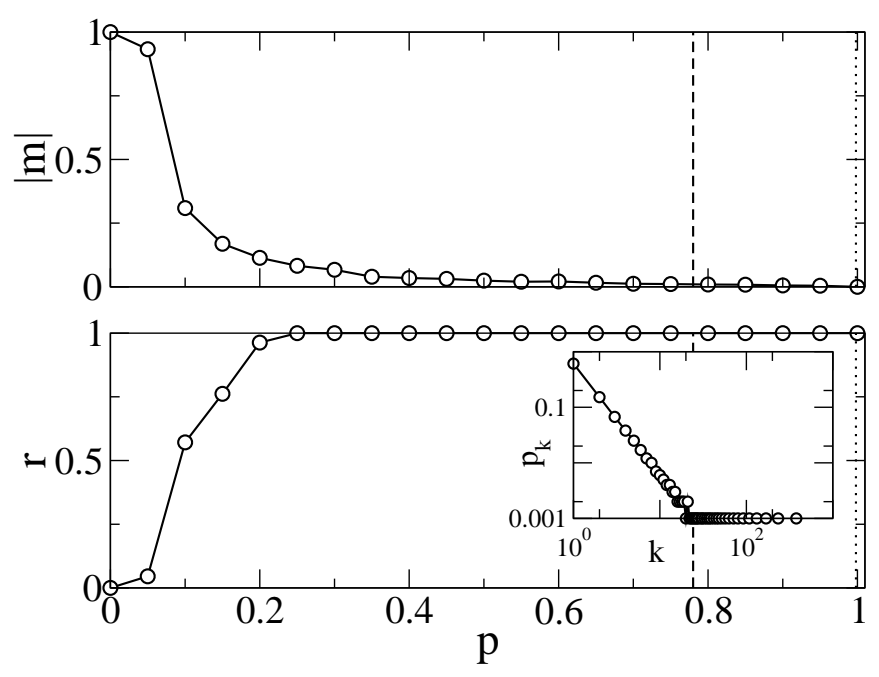

Figure 5.10.: Early fragmentation in scale-free networks. The plots are analogous to Fig. 5.9 but describe networks with the out-degree distribution $p_{k} \propto k^{-2}$ (inset). Fragmentation occurs far below the estimated transition points (dashed, dotted) and extends over a wider range. $N=10^{4}$, $\langle k\rangle=5.5665, \hat{k}_{\text {in }}=\hat{k}_{\text {out }}=10$.

p. Considering individual simulation runs in detail, one finds that the networks remain for some time in an active state before slowly approaching fragmentation-a behaviour not observed in the undirected model or in the directed networks considered in the previous section.

The observation that the networks spend some time in the active state before fragmenting indicates that these states are still feasible at least in the beginning of the simulation runs. The mechanism by which fragmentation is reached must therefore differ from the mechanism observed in the cases studied so far, where fragmentation occurs due to the destabilization of the parabola of active states in a transcritical bifurcation.

Notably both the moment and motif expansion seem not to capture the different mechanism for fragmentation because they significantly overestimate the fragmentation point. The main assumption used in both approximations is the absence of correlations between a node's in- and out-degree and between nearest neighbours. Their failure thus indicates the appearance of correlations that are absent or not substantial in the networks with Poissonian out-degree distribution. In the following we call fragmentation well below the estimated fragmentation point early fragmentation.

Network simulations suggest that early fragmentation is initiated by the formation of self-stabilizing structures among the agents. To understand the process leading to such structures, consider that the networks, even far from fragmentation, are partially ordered. In average, the number of in- and outgoing neighbours of an agent that share the focal agent's opinion will be greater than the number of neighbours that oppose the focal agent's opinion, because the rewiring dynamics transforms active links into inert ones. This 


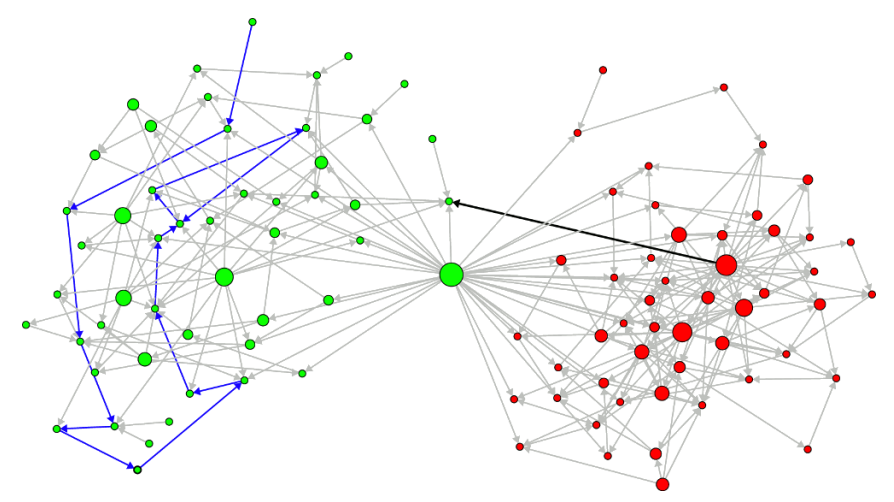

Figure 5.11.: Almost fragmented network of $N=100$ nodes with out-degree distribution $p_{k} \propto k^{-2}$. The two components are only connected by the hub node with the largest out-degree and a single link from the second-to-largest hub (black line). A self-stabilizing triangle of nodes of out-degree one and a subsequently recruited stable "chain" of nodes of out-degree one are marked in blue. Note that the nodes with high out-degree have very low or zero in-degree.

implies that if an agent changes her opinion, she is likely to experience a subsequent loss of incoming links because the majority of her neighbours now oppose her opinion and rewire their links with some probability. In the long run, agents that frequently change their opinion have lower in-degree than those who change their opinion rarely. Therefore, the attention, measured in terms of incoming links, focuses on the agents that have a low out-degree and thus rarely change their opinion.

Focusing the attention on agents of low out-degree impedes the propagation of opinions across the network. In particular, it can lead to the formation of small clusters which have few outgoing links and hence have a very high resistance to invasion of the opposing opinion. In an extreme case, small subgraphs can form in which all nodes are in consensus and all outgoing links starting within the subgraph lead to other nodes in the same subgraph. Because of this lack of outgoing links, such subgraphs can never be invaded by the opposing opinion. Furthermore, no outgoing links leaving the subgraph can be formed because none of the nodes in the subgraph will ever rewire an outgoing link.

We call subgraphs that are hard or impossible to invade self-stabilizing structures. The initial formation of such a structure is a stochastic event that occurs with a small probability. However, once such a structure has formed, it can grow as other nodes rewire their outgoing links into the structure. Nodes of low out-degree can be recruited rapidly because only few rewiring events are necessary to rewire all of their outgoing links into the self-stabilizing structure. Recruitment of nodes with more outgoing links takes longer because more rewiring events are required for this. In simulations, networks observed shortly before fragmentation are often found to consist of two almost disconnected clusters, which are only connected by a few nodes of high out-degree. Because of their frequent changes of state, these connecting hubs have very few or no incoming links.

For illustration of the mechanism described above, an embedding of a small network 


\section{Opinion formation}

shortly before fragmentation is shown in Fig. 5.11. The network has broken into two almost disconnected clusters. The remaining connections are formed by a single hub and one additional link. The fragmentation has been nucleated by the formation of a self-referential cycle consisting of three nodes of out-degree one. Subsequently, almost half of the network has been recruited into this self-stabilizing structure.

Given the observations above, we can explain the shape of the trajectories shown in Fig. 5.7. Because the formation of a self-stabilizing structure is a rare event, they are generally not present in the initial network. The system therefore approaches the parabola of active states, which is in agreement with results from the undirected model and the analytical approximations for the directed model. However, while the system drifts along the parabola of active states, self-stabilizing structures are eventually formed due to the ongoing rewiring. As the self-stabilizing structures grow, the permissible range for the magnetization shrinks, effectively arresting $m$ as almost all nodes are recruited into the self-stabilizing structures. Because the last nodes to join the structures are "hub" nodes with high out-degree, a relatively high density of active links can be maintained for some time. Because the hub nodes undergo rapid opinion switches, rewiring can only slowly separate them from opposing neighbours, which explains the slow fragmentation. The switching and rewiring of the hub nodes are clearly visible in the time series of the active link density $\rho$. In the inset in Fig. 5.7 this is shown for the last $10^{3}$ time units before fragmentation. Here, one of the two remaining connecting hubs detaches from one of the components and the final hub still switches several times before eventually also separating.

Summarizing the observations above, we conjecture that early fragmentation is initiated by the formation of self-stabilizing structures among nodes of low out degree. We emphasize that contrary to most dynamical phenomena observed in scale-free networks, the dynamics of interest is generated primarily in the nodes of low degree. Nodes of high degree still play an important role as they are the last nodes to connect the separating components and thus determine the time of fragmentation. This mechanism is not captured by current analytical approaches, because it relies on the build-up of negative correlations between the in-degree and out-degree of nodes that is neglected in the approximation schemes.

The important role of the nodes with low out-degree becomes obvious if one considers networks in which a lower bound on the out-degree is enforced. We construct networks with a minimum out-degree of $k_{\text {out }}=2$ from scale-free networks by randomly redistributing outgoing links to the nodes with out-degree $k=1$ until every node has at least two outgoing links. Clearly, the resulting networks cannot be called scale-free. Nevertheless, they still exhibit a broad out-degree distribution and contain a few hub nodes of very large out-degree. The results in Fig. 5.12 show that in such networks a relatively sharp fragmentation transition is observed again. This is because self-stabilizing structures are formed less frequently in these networks, as they are mostly created by rewiring among the nodes with out-degree two now, which must rewire both their outgoing links into a forming structure to stabilize it. 


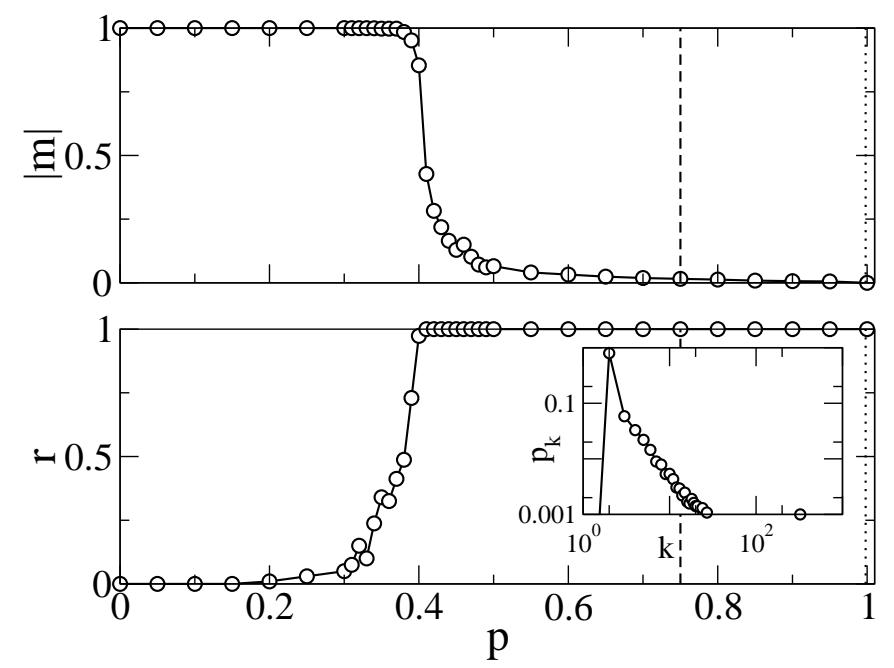

Figure 5.12.: Fragmentation in networks with an out-degree distribution roughly following $p_{k} \propto$ $k^{-2}$, in which a minimum out-degree of $k_{\min }=2$ is enforced by redistributing outgoing links to the nodes with $k_{\text {out }}=1$ (inset). Compared to scale-free networks, a relatively sharp transition is again observed at a larger critical rewiring rate, which is still overestimated by the analytical predictions. $N=10^{4},\langle k\rangle=5.5665, \hat{k}_{\text {in }}=\hat{k}_{\text {out }}=10$.

Although the nodes with out-degree one play an important role for early fragmentation, they alone cannot explain this phenomenon. The motif expansion still overestimates the fragmentation point significantly (Fig. 5.12), even though the modified out-degree distribution can be taken into account in this approach. Therefore, the emerging correlations between the nodes' in- and out-degrees, which are neglected in the approximations, are of similar importance.

\section{Poissonian out-degree distribution with low $\langle k\rangle$}

Because the mechanism postulated above relies on the formation of correlations, one can perform a simple test by considering a system in which these correlations are removed by an additional rewiring process. However, such a test is for two reasons difficult in scalefree networks: First, because of the constraints in scale-free topology it is well-known that it is difficult to remove correlations in scale-free networks completely, and second, because of the presence of nodes of very high degree, fragmentation takes a long time, making numerical studies of fragmentation tedious.

Our reasoning above predicts that early fragmentation should be observed also in directed Poissonian networks with sufficiently low mean degree. In the present section we therefore consider a Poissonian network with a mean degree of 4 , which avoids the difficulties encountered in scale-free networks. In this section we show a) that this network exhibits early fragmentation and $b$ ) that the early fragmentation can be avoided 

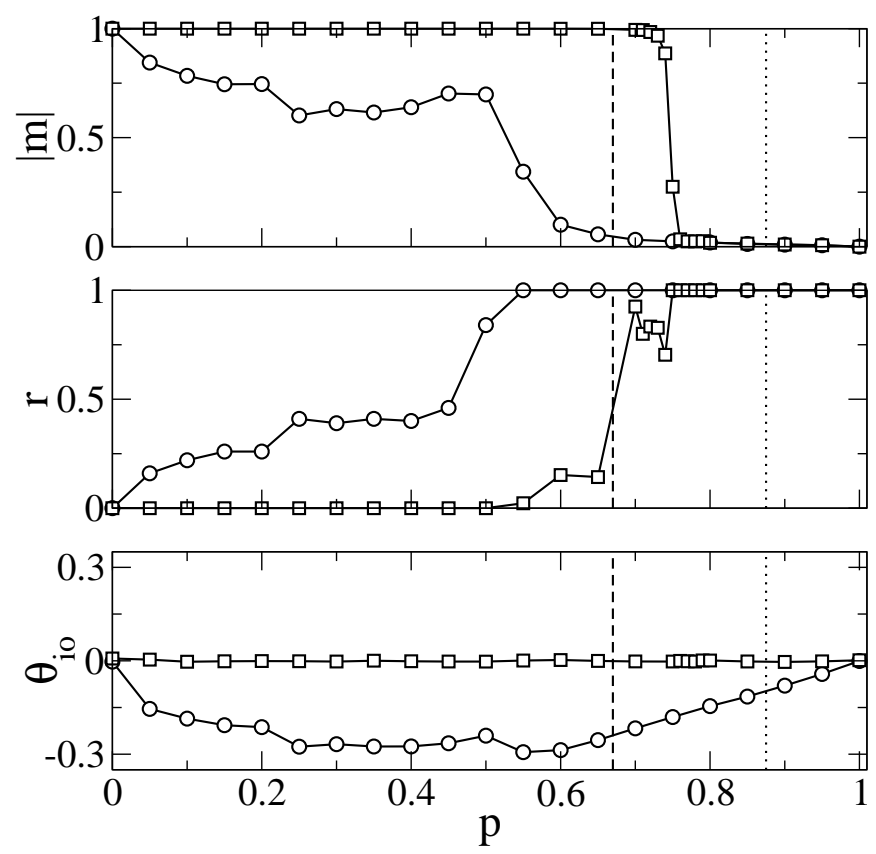

Figure 5.13.: Fragmentation of networks with Poissonian out-degree distribution and low $\langle k\rangle$, in which the inert links are also rewired (squares) or are not rewired (circles). Early fragmentation is clearly visible, although the shown averages over 100 simulation runs are still rather noisy due to the highly stochastic nature of early fragmentation. Shown are the absolute value of the magnetization (top), the proportion of fragmenting simulation runs (centre), and the correlation coefficient between the in- and out-degree of the nodes in the final state (bottom). $N=10^{4}$, $\langle k\rangle=4, \hat{k}_{\text {in }}=\hat{k}_{\text {out }}=10$.

by an additional rewiring mechanism that destroys the correlations implicated in the formation of self-stabilizing structures.

Simulation results for the network described above are shown in Fig. 5.13. The figure shows clear evidence of fragmentation well below the estimated fragmentation point. Further, this early fragmentation is accompanied by the build-up of negative correlations between the in- and out- degrees of the nodes, which is measured by their correlation coefficient 7 This confirms our previous observation that attention focuses on those nodes who pay little attention to others themselves.

To verify that the correlation described above is the cause and not a symptom of the early fragmentation, we now consider a different variant of the model. This variant is identical to the model used so far, except that when an inert link is chosen, this link is also rewired to a randomly chosen target node that is in the same state as the source.

${ }^{7}$ The linear correlation coefficient, or Pearson product-moment correlation coefficient, between the in- and out-degree of the nodes is defined as $\theta_{\text {io }}=\left\langle\left(k_{\text {in }}-\langle k\rangle\right)\left(k_{\text {out }}-\langle k\rangle\right)\right\rangle / \sigma_{\text {in }} \sigma_{\text {out }}$, where $\sigma_{\text {in }}$ and $\sigma_{\text {out }}$ denote the standard deviations of the in- and out-degrees, respectively. 
The model variant in which the rewiring of inert links is switched on shows no evidence for early fragmentation (see Fig. 5.13). Fragmentation occurs in a relatively sharp transition at a critical rewiring rate $p_{c}$ that is consistent with the estimate from the motif expansion. We emphasize that the rewiring of inert links neither introduces nor destroys active links. It therefore has no direct impact on fragmentation. However, rewiring inert links prevents the build-up of correlation between the in-degree and the out-degree of nodes and thereby inhibits the formation of self-stabilizing structures. The absence of early fragmentation in a model where these correlations are removed confirms the causal relationships postulated above. We therefore conclude that in directed adaptive networks, the slow build-up of negative correlations between in-degree and out-degree can initiate early fragmentation by leading to the formation of self-stabilizing structures.

\subsubsection{Conclusions}

In this section, we have investigated an extension of the $\mathrm{VM}$ on adaptive networks that takes the directionality of inter-agent interactions into account. We found that our model can transition to a fractionated state for rewiring rates that lie much below the critical value estimated using analytical approaches that are known to work well in the undirected case. We discovered that fragmentation occurs due to a novel mechanism that depends inherently on the directed nature of the links. This early fragmentation occurs when agents focus their attention on those who are steady in their opinion because they pay attention only to few sources of information. In this case, self-stabilizing structures can form that nucleate fragmentation.

The mechanism described here might constitute a threat to one of the central functions of real-world opinion formation processes, namely the debunking of counter-factual opinions. The formation of self-stabilizing structures in our model would correspond to a situation where a given subset of the society only pays attention to information sources with the same belief system, thus reinforcing and perpetuating opinions that are never confronted with opposing views. Recent media reports have indeed pointed out the existence of similar self-stabilizing structures in political media networks in the U.S. (Maddow, 2010).

Strong homophily, i.e. the tendency of humans to favour discussing with others who share similar beliefs, seems to be facilitated by current sociological and technological developments. With the increasing diversity of offline and online media (Webb, 2006. Flaounas et al. 2010) and new media technologies (Hensinger et al. 2010), it is becoming easier to avoid opposing opinions altogether. In particular, the Internet enables people not only to access but also to publish information easily. One of the best examples is perhaps the micro-blogging service Twitter, which hosts more than a billion user posts per week $\left.\right|^{8}$ Among this flood of information, it is easy to find sources supporting almost every conceivable opinion, while avoiding contradicting evidence.

\footnotetext{
${ }^{8}$ as reported at http://blog.twitter.com/2011/06/200-million-tweets-per-day.html
} 


\section{Opinion formation}

In this light, the potential existence of an early fragmentation mechanism may be both problematic and an opportunity. Early fragmentation maintains the diversity of opinions: it may thus aid the survival of counter-factual myths, but also of legitimate and well-founded views of minorities.

On more general grounds, our results illustrate that directed networks can exhibit new physics not observed in their undirected counterparts. Especially in the investigation of opinion formation processes, the often directed flow of attention should therefore be taken into account in models.

The adaptive directed VM still paints a highly simplified picture of real-world opinion formation processes and thus must be considered as a toy model. Therefore, investigation of more realistic models is an important goal for the future. Based on the results and analysis presented here, we believe that the mechanism of early fragmentation will be observed whenever directed attention is focused preferentially on agents that change their opinions at less than average rate. We therefore expect that early fragmentation should be robust to future refinements of the model.

A key ingredient that is missing in our present model is novelty. Here we considered only the exchange of opinions regarding a single, well-defined question, whereas in reality many discussions are enriched by the constant inflow of new ideas. We have shown that homophily favours connecting to poorly informed agents and thereby promotes early fragmentation, whereas curiosity would favour connecting to well informed agents and thereby hinder early fragmentation. In this light, novelty, whether in the form of true innovation or arbitrarily changing fashions may play an important role in preventing social fragmentation.

\subsection{Summary}

In this chapter, we have studied opinion and consensus formation in populations of interacting agents. In the paradigmatic adaptive VM, agents holding a binary opinion interact with other agents in an adaptive network, in which they can adopt their neighbour's opinions or rewire their connections to other agents. We have seen that the coupling of the topological dynamics to the node dynamics can lead to a global reorganization of the topology in this model, ultimately causing the fragmentation of the network into at least two disconnected components. Fragmentation occurs if the time scale ratio of opinion adoption and rewiring exceeds a critical threshold, which is well approximated by a motif expansion method.

Although the approximate analytical description of the adaptive $\mathrm{VM}$ in terms of moment equations overestimates the critical rewiring rate, it allows to understand qualitatively the mechanism leading to fragmentation. At the critical rewiring rate, above which fragmentation is observed, the active, non-fragmented stationary states become unstable in a transcritical bifurcation. Above this point, the fragmented states are the only accessible and stable stationary states, so that fragmentation is always observed. Fragmentation is 
thus enabled by the destabilization of the active stationary states in the adaptive VM in undirected networks.

We proposed a novel extension of the adaptive $\mathrm{VM}$ to directed networks. This directed adaptive VM allows to consider the effect of asymmetric inter-agent interactions on opinion formation and study the influence of different out-degree distributions. The fragmentation transition known from the undirected case is also observed in directed networks with Poissonian out-degree distributions and a sufficiently large average degree. The analytical approaches used for undirected networks can also be applied in this case and yield similar results.

In networks where a significant number of nodes has low out-degree, however, a novel phenomenon is observed. In such networks, the rewiring dynamics can lead to the formation of self-stabilizing structures in our model. These structures nucleate the formation of stable consensus groups and thus promote the fragmentation of the network. As we have observed this type of fragmentation at rewiring rates far below the fragmentation point estimated by the motif expansion approach, we call it early fragmentation. In contrast to "ordinary" fragmentation that depends on the destabilization of the active stationary states at large rewiring rates, early fragmentation is initiated by a different mechanism, namely the formation of self-stabilizing structures. This mechanism cannot be accounted for in the macroscopic ODE description, because the formation of such structures is an inherently microscopic, i.e. localized phenomenon.

The topological transition from a connected network of agents holding different opinions to a fragmented network consisting of disconnected, state-homogeneous components is an important example of the phenomena genuinely associated with adaptive networks. Here, a complex topology is achieved from an initially random configuration through the tight coupling between local and topological degrees of freedom. For ordinary fragmentation, this can be interpreted as a dynamical phase transition in which the fragmented network state becomes stable. Early fragmentation, on the other hand, is possible because the adaptive nature of the network allows for the formation of self-stabilizing structures. 



\section{Evolution of cooperation}

In this chapter, the concept of adaptive networks is applied in the field of evolutionary game theory, which is concerned with the question how cooperative behaviour can evolve and persist in a population of inherently selfish individuals. We will see how the dynamical self-organization in adaptive networks can act as a mechanism promoting cooperation. Specifically, I discuss an adaptive-network model of interacting agents in which the topological response to the agents' local dynamics gives rise to oscillatory behaviour and allows for asymptotically full cooperation. I show how this novel dynamical mechanism can be understood from the emergent-level ODE description.

\subsection{Cooperation in nature and society}

The phenomenon of cooperation is ubiquitous in nature. It can be found on a wide range of scales: in genes that cooperate in forming and controlling cells; bacteria and other microbes that show cooperative behaviour; cells that cooperate in multicellular organisms; and animals that cooperate in social groups (Kappeler and van Schaik, 2006. West et al. 2007. Pennisi 2009). Clearly, cooperation is at the heart of human society. Humans cooperate in families, neighbourhoods, and cities forming countries and states. States cooperate in global politics. Firms cooperate in joint ventures and global markets. Thus, to a large extent, human behaviour is cooperative behaviour.

The evolutionary success of the human species is built on cooperation, and the stability of human society hinges on its maintenance. Yet, how cooperative behaviour evolves and is maintained among inherently selfish individuals is still not fully understood, nor are the mechanisms behind the sometimes catastrophic failures of cooperation in human society (Turchin 2003).

Cooperative behaviour is a behaviour of an individual that provides a benefit to others and comes at a cost to the individual. In many situations, the cooperating individual itself (the cooperator) will also benefit from its behaviour, although this does not have to be the case. By providing a benefit to others, a cooperator faces the risk of being exploited by other individuals who only use the benefit but do not contribute anything themselves. Thus, these individuals (the defectors) do not have to spend resources on the costly cooperative act, which allows them to enjoy the full benefit.

A specific example found in social animals is alarm call behaviour. In many animals living in groups, the group members take turns in watching out for predators and giving alarm calls while the others are foraging or resting. Thus, these sentinels put themselves at 


\section{Evolution of cooperation}

risk for the benefit of the rest of the group ${ }_{1}^{1}$ Group members that never stand sentinel can avoid this risk and enjoy the benefit of being warned of potential danger, thus exploiting the sentinel individuals.

This example illustrates the key question in the evolution of cooperation: If cooperation is costly and prone to exploitation, why is it so common in nature and society? How does cooperation evolve among self-interested individuals? This is a long-standing question in evolutionary biology, where it can be phrased in rather well-defined words: If costly cooperation can be exploited by defectors so that they can outcompete cooperators, how can it evolve under natural selection? This central question has been addressed in numerous works using the framework of evolutionary game theory.

Evolutionary game theory uses concepts from economics and game theory to describe the evolutionary dynamics of populations of interacting agents, such as genes, microbes, and animals. It has contributed many valuable insights to our current understanding of the genetic evolution of cooperation. This term refers to the evolution of biological species, whose key ingredients are reproduction, mutation, and natural selection. Furthermore, evolutionary game theory may also be used to study the cultural evolution of social behaviour, which refers to the evolution of cultural traits in a population of social agents, e.g. humans, that can imitate each other.

Clearly, applying an abstract and highly simplified theory to as complex a system as human society needs to be done with delicate caution. Still, keeping in mind the assumptions that determine the scope of its results, evolutionary game theory is a valuable tool to understand the basic principles behind the evolution and possible failure of cooperation in social systems (Frank, 2009).

In this chapter, I give a brief introduction to the basic concepts of evolutionary game theory and its application to cultural evolution in social systems. After discussing heterogeneous interactions as a key mechanism promoting cooperation, I present a model for the evolution of cooperation in adaptive networks. In this model, the coupling between evolutionary and topological dynamics gives rise to a novel dynamical mechanism leading to asymptotic full cooperation.

\subsection{Evolutionary game theory}

The phenomenon of cooperation, its emergence and survival not only in biological systems but also in a social and economic context, can be investigated in the framework of evolutionary game theory. Since its beginnings (Maynard Smith and Price, 1973. Axelrod and Hamilton, 1981: Maynard Smith, 1982, Axelrod, 1984), it has attracted considerable attention from scientists in a variety of fields. It uses concepts from mathematical game

${ }^{1}$ It has been argued that sentinel behaviour is not an altruistic (i.e. selfless) act but rather a selfish behaviour (Bednekoff 1997. Blumstein 1999). As considered in the present work, however, cooperative behaviour is not required to contain an altruistic element. Standing sentinel and giving alarm calls therefore is an example of cooperation in animal groups, whether it is altruistic or not. 
theory to model the interactions between individuals. This allows to define the evolutionary success of a behavioural type, which results from these interactions, in terms of the outcome, or payoff, of a well-defined game (Weibull, 1997).

In order to discuss evolutionary game theory, some basic concepts from mathematical game theory are needed. Game theory was originally invented by von Neumann and Morgenstern (1944) as a "theory of economic behaviour" that deals with decision making of interacting rational individuals. A concise characterization of game theory is given by Ross, who defines it as

[...] the study of the ways in which strategic interactions among economic agents produce outcomes with respect to the preferences (or utilities) of those agents, where the outcomes in question might have been intended by none of the agents. (Ross, 2010)

Game theory thus is a theory of decision making in the interactions with others. It assumes the game players to act rationally, using all the information they can access to maximize their utility, which is an abstract measure for the agents' preferences. In a bargaining situation, for instance, the seller's utility corresponds to the money he makes by selling an item, whereas the buyer's utility could be the money the buyer saves. Placing strategic offers, both try to maximize their utility. The seller wants to achieve the highest possible price that sells the item. The buyer, on the other hand, wants to pay the smallest possible price that buys the item, i.e. save the highest possible amount of money. In this situation, whether the item is sold and at what price depends on the bargaining skills of both participants, and so does the utility of both players.

To provide some insight into the type of strategic situations considered in game theory, I discuss the famous Prisoner's Dilemma in the next section.

\subsubsection{The Prisoner's Dilemma}

The basic ideas of game theory are best explained using the well-known Prisoner's Dilemma (PD) game, which is typically illustrated by the following narrative. Imagine two suspects being arrested for having jointly committed some crime. Evidence, however, is insufficient for a conviction, so the police speak separately to the prisoners, offering both the same deal: If one testifies against his accomplice and the other remains silent, the former goes free while the latter receives the full sentence of ten years in prison. If both remain silent, each of them is sentenced to two years only, whereas both receive five years in prison if they testify against each other. The prisoners are not allowed to confer, and they must choose either to testify or to remain silent.

The situation can be summarized in a table of possible outcomes (years in prison) for each prisoner:

\begin{tabular}{l|ll} 
& B silent & B testifies \\
\hline A silent & 2 years each & B free, A 10 years \\
A testifies & A free, B 10 years & 5 years each
\end{tabular}




\section{Evolution of cooperation}

Assuming that both prisoners decide rationally and want to minimize their own time in prison, it is immediately clear that they should always testify, no matter what the other does. If B remains silent, A should testify in order to be set free. Conversely, if B testifies, A should again testify, because he will then serve only five years in prison, which is preferable to serving ten years when remaining silent. The same reasoning can be applied for prisoner B. Thus, rational prisoners will always choose to testify against their accomplice in this situation, which, however, results in five years in prison for each of them.

The crucial point in the $\mathrm{PD}$ game is that the prisoners could minimize the total time they spend in prison if both remained silent, in which case they would serve only two years each. Yet, the individual prospect of going free by testifying against the other prevents this outcome and leads to the suboptimal result of a five-year sentence. Therefore, this game is called a dilemma ${ }^{2}$

In the language of game theory, the $\mathrm{PD}$ can be formalized as a strategic game played by two players choosing simultaneously from two strategies. ${ }^{3}$ namely cooperation (C) and defection (D). Both players receive a (possibly abstract) payoff that depends on their strategy and the strategy of their opponent. In the situation described before, remaining silent corresponds to cooperation and testifying to defection. The payoff (counted positive) of one player corresponds to the number of years not spent in prison compared to the maximum sentence of ten years. The game is then fully described by its payoff matrix,

$$
\begin{gathered}
C \\
D \\
D
\end{gathered}\left(\begin{array}{cc}
8 & 0 \\
10 & 5
\end{array}\right),
$$

giving the payoff received by the row player in dependence of the strategy chosen by the column player. Again, a rational row player trying to maximize his payoff should always choose $\mathrm{D}$, because a payoff of 10 is preferable to 8 and a payoff of 5 is preferable to 0 . Both players choosing to defect is the only Nash equilibrium in the PD game. This means that none of the players can increase their payoff by choosing to cooperate, given that the other defects (Osborne and Rubinstein, 1994). By contrast, if the other cooperates, defecting always increases a player's payoff.

In general, the $\mathrm{PD}$ game is defined in its normal form by a payoff matrix

$$
\begin{gathered}
C \\
C \\
D
\end{gathered}\left(\begin{array}{ll}
R & S \\
T & P
\end{array}\right),
$$

where the matrix entries are traditionally named "reward" $R$ for mutual cooperation,

\footnotetext{
${ }^{2}$ Much can be said about the philosophical and ethical implications of the $\mathrm{PD}$ and the assumption of rational individuals, which is beyond the scope of the present work. I refer the reader to, e.g., Lucas (1959) or Cunningham (1967).

${ }^{3}$ Two-player games with two possible strategies are called $2 \times 2$-games.
} 
"sucker's payoff" $S$, “temptation to defect" $T$, and "punishment for mutual defection" $P$. The ordering $T>R>P>S$ defines the $\mathrm{PD}$ game, ensuring that unconditional defection is always the best strategy. Because of this ordering, the $\mathrm{PD}$ game constitutes a "social dilemma," as the defecting players receive a total payoff that is lower than if they had both cooperated. Different situations can be described by (6.1) by choosing different relative orderings of the matrix entries.

In order to reduce the number of free parameters, alternative parametrizations of the payoff matrix are often considered. A common parametrization for the $\mathrm{PD}$ game is the benefit-cost-matrix

$$
\begin{gathered}
C \\
C \\
D
\end{gathered}\left(\begin{array}{cc}
b-c & -c \\
b & 0
\end{array}\right),
$$

which has a very intuitive interpretation: cooperators invest a cost $c$ in the cooperative act providing a benefit $b$ to others, whereas defectors do not. For $b>c>0$, this is a special case of (6.1) that implies the restriction $R+P=S+T$.

\subsubsection{Strategic games and evolution}

Evolutionary game theory combines formal games like the $\mathrm{PD}$ game with population dynamics, i.e. dynamics of reproduction. In a population of agents, the agents have different behavioural strategies or traits (phenotypes) that determine their performance in the interaction with others and their environment. The performance of an agent in turn determines its ability to reproduce. In other words, agents with a behavioural strategy or trait that performs well in the interaction with others have a higher probability to reproduce. Hence, the behavioural success of an agent defines its evolutionary fitness, i.e. its reproductive success.

In evolutionary game theory, the interactions between competing agents are represented by formal games, where the agents' different behavioural strategies are mapped to the possible game strategies. The success of an agent is then simply the payoff it obtains from the games played with other agents, and the fitness of an agent is a function of its success. Agents whose strategy performs well in the game interaction can thus proliferate, possibly outcompeting the other agents.

The key concepts of the theory of genetic evolution can be carried over to cultural evolution, if behavioural traits are replaced by behavioural strategies, and reproduction is replaced by imitation. In genetic evolution, the frequency of a particular phenotype changes due to the reproduction of successful agents. In cultural evolution, on the other hand, the frequency of a behavioural strategy changes due to the imitation of successful agents. Although essentially different, genetic and cultural evolution are rather similar from the modeller's point of view. Both can be considered as the reproduction and spread of traits (strategies) in a population of agents striving for success. For this reason, genetic and cultural evolution can often be studied in technically equivalent models, paying due attention to the precise interpretation of the results. 


\section{Evolution of cooperation}

As an example, consider a large population of cooperators and defectors who interact in random pairs in a $\mathrm{PD}$ game according to the payoff matrix 6.1). The population is assumed to be well-mixed, so that every agent interacts with every other agent with the same probability. Thus, all agents following the same strategy obtain the same payoff. If we consider reproduction dynamics (genetic evolution), we assume that the reproduction rate of an agent is given directly by its payoff. For imitation dynamics (cultural evolution), we assume that an agent's rate of being imitated is given by its payoff. In both cases, the proportion $x$ of cooperators in the population changes in time according to ${ }^{4}$

$$
\dot{x}=x\left(f_{C}-\bar{f}\right),
$$

where $f_{C}$ is the total payoff of a cooperator and $\bar{f}=x f_{C}+(1-x) f_{D}$, the average payoff of the population. With the notation of (6.1), $f_{C}=x R+(1-x) S$ and $f_{D}=x T+(1-x) P$. Equation (6.3) is exact in the limit of infinite population size and well-mixed populations. It is a special case of the replicator equation,

$$
\dot{x}_{i}=x_{i}\left(f_{i}(\mathbf{x})-\bar{f}(\mathbf{x})\right),
$$

which describes the evolution of the relative abundance of strategy $i$ in a well-mixed population of agents with $i=1, \ldots, n$ strategies under frequency-dependent selection (Hofbauer and Sigmund 1998). Here, $f_{i}(\mathbf{x})$ denotes the fitness of agents following strategy $i$, which is a function of the frequencies of all strategies in the population, and $\bar{f}(\mathbf{x})$ is its population average. From (6.4) it is clear that agents whose fitness is above average will thrive, which formalizes Darwinian survival of the fittest.

If the payoff matrix satisfies the ordering $T>R>P>S$ of the $\mathrm{PD}$ game, the only stable stationary state of (6.3) within the physical bounds $0 \leq x \leq 1$ is the state containing zero cooperators, $x^{*}=0$. Hence, assuming that the agent interactions can be modelled as $\mathrm{PD}$ games, evolutionary game theory predicts that cooperation should not evolve and survive under natural selection in well-mixed populations. Therefore, additional mechanisms must exist that allow cooperators to thrive despite their evolutionary disadvantage of being prone to exploitation. I discuss examples of such mechanisms in section 6.3 specifically focusing on network reciprocity.

\section{Other 2×2-games}

The payoff matrix (6.1) does not only describe the $\mathrm{PD}$ game. Depending on the relative ordering of the matrix entries, it also captures three other paradigmatic games, namely the Snowdrift game, the stag-hunt game, and the harmony game. Depending on the underlying game, the replicator equation (6.3) has different stable and unstable fixed points (Fig. 6.1).

The ordering $T>R>S>P$ describes the Snowdrift (SD) game, in which mutual defection is no longer the only outcome. It models a situation in which it is the best to

\footnotetext{
${ }^{4}$ This equation is derived in appendix $\mathrm{C}$ for both reproduction and imitation dynamics.
} 


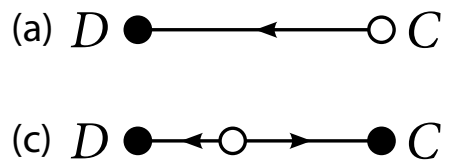

(a) $D$

(c) $D$

Figure 6.1.: The four different $2 \times 2$-games in well-mixed populations: (a) only full defection is stable in the $\mathrm{PD}$ game, (b) only coexistence of cooperators and defectors is stable in the $\mathrm{SD}$ game, (c) full defection and full cooperation are stable in the stag-hunt game, and (d) only full cooperation is stable in the harmony game.

do the opposite of what one's opponent does. As the $\mathrm{PD}$ game, it is typically illustrated using a short narrative: A car with two passengers is stuck in a snowdrift. To be able to drive on, the passengers need to remove the snowdrift blocking their way. Now, each passenger has two options: get out of the car and start shovelling (i.e. cooperate), or stay in and wait for the other to do all the work (i.e. defect). If both shovel, they can share the work and be on their way again in a short time. If only one shovels, they can still go on eventually, whereas they will not be able to go on if both stay in the car. The best choice for each player in the SD game thus depends on the action of the other. If the opponent cooperates (shovels), it is best to defect (stay in the car and wait). If the opponent defects (stays in), it is best to cooperate (shovel).

The $\mathrm{SD}$ game is also called hawk-dove game or chicken game, depending on the situation it is used as a metaphor for (Sugden, 1986. Doebeli and Hauert, 2005). Similar to the PD game, the $\mathrm{SD}$ game is often studied in a reduced form using the benefit-cost-matrix

$$
\begin{gathered}
C \\
C \\
D
\end{gathered}\left(\begin{array}{cc}
b-\frac{c}{2} & b-c \\
b & 0
\end{array}\right),
$$

which emphasizes that the players share the cost $c$ if both cooperate.

In contrast to the $\mathrm{PD}$ game, cooperators in a well-mixed population interacting in the $\mathrm{SD}$ game have an advantage when rare. Therefore, cooperation does not die out under $\mathrm{SD}$ dynamics. Rather, equation (6.3) has an interior stable fixed point at $x^{*}=1-c /(2 b-c)$ in this case $:^{5}$ so that an intermediate level of cooperation is maintained (Fig. 6.1 b). It is important to note, however, that the $\mathrm{SD}$ game still constitutes a social dilemma, because the total payoff of the population would be greater if everybody cooperated. Nevertheless, in evolutionary game theory, the $\mathrm{SD}$ has received only little attention, because it does not present such a difficult situation for cooperators as the $\mathrm{PD}$ game and already allows for a moderate level of cooperation without any additional mechanisms. Still, it is considerably difficult to measure the payoff matrix for, e.g., the interaction of different species, so that it is not clear whether real-world interactions are best modelled using the $\mathrm{PD}$ or the $\mathrm{SD}$ game (Doebeli and Hauert, 2005). In fact, the SD game might be more appropriate for

\footnotetext{
${ }^{5}$ For the more general payoff matrix 6.1 with SD game ordering, $x^{*}=(P-S) /(P-S+R-T)$.
} 


\section{Evolution of cooperation}

modelling the competition between different microbial strains (Gore et al. 2009), and in particular for modelling the cooperation among humans (Kümmerli et al. 2007).

The ordering $R>T>P>S$ describes the stag-hunt, or coordination game. In this game, it is the best for each player to choose the same strategy as the other. Only mutual cooperation provides the highest payoff for both, but a player also faces the highest risk when choosing to cooperate, namely the least payoff $S$ if the other defects. This game is named after the following narrative: Two hunters go on a hunt together, each choosing to hunt for a stag (cooperate) or a hare (defect) without telling the other. A single hunter can get a hare by himself, but they can only get a stag if working together. As a stag is worth more than a hare, choosing to defect minimizes a players risk but choosing to cooperate opens the possibility of obtaining a bigger reward, namely the stag. For the stag-hunt game, the replicator equation has two stable fixed points, $x_{0}^{*}=0$ and $x_{1}^{*}=1$, whereas the single interior fixed point $x^{*}$ is unstable (Fig. 6.1 c). The dilemma in this game is that cooperation can only be maintained if at least a fraction $x>x^{*}$ of the population cooperates, which then leads to full cooperation. If most of the players prefer to minimize their risk by initially choosing to defect, however, cooperation will die out.

For the sake of completeness, let us consider the ordering $R>T>P \wedge R>S>P$. It describes the harmony game, in which cooperation is always the best choice. Full cooperation is the only stable fixed point of the replicator equation in this case (Fig.6.1 d). Therefore, this game does not present a social dilemma, as its outcome is always mutual cooperation.

\subsection{Mechanisms promoting cooperation}

A first and fundamental prediction of evolutionary game theory is that under PD-type interactions, cooperation should not evolve, unless some additional mechanism works in favour of cooperators. Many different such mechanisms have been identified in the last decades (Hofbauer and Sigmund, 1998 Hammerstein, 2003 Levin, 2009). I briefly summarize some of them in the next paragraphs, roughly following Nowak's 'five rules for the evolution of cooperation' (Nowak, 2006).

One important mechanism promoting cooperation is kin selection, which is particularly relevant for genetic evolution. It refers to the idea that cooperation can be promoted by natural selection if the interacting agents are genetic relatives. This is formalized in Hamilton's rule stating that cooperation is favoured if $r>c / b$, where $r$ measures the relatedness of the agents in terms of the probability of sharing a gene, and $c / b$ is the cost-to-benefit ratio of the $\mathrm{PD}$ according to (6.2) (Hamilton, 1964. West et al. 2002). Kin selection provides an explanation for many examples of cooperation observed in biology, for instance in social insects (Bourke and Franks, 1995. Ratnieks et al. 2001).

Cooperation among humans, however, goes far beyond the scope of relatives. In fact, in many situations we are even inclined to help complete strangers without any immediate benefit to ourselves. Thus, while kin selection is highly relevant in the evolution of 
biological species, there must be other mechanisms at work in cultural evolution that do not necessarily rely on relatedness. The most relevant such mechanisms depend on some form of reciprocity.

Direct reciprocity emerges if the same agents interact more than once and remember the previous interactions. Cooperation can thus prevail, because "if I help you, you might help me later." Indeed, when the $\mathrm{PD}$ game is played repeatedly between the same two players, always defecting is no longer the strategy that ensures the highest average payoff. Instead, this is achieved by tit-for-tat, a strategy that cooperates in the first round and then always does whatever the opponent did in the previous round (Axelrod and Hamilton, 1981). An even more successful and robust strategy is win-stay, lose-shift, in which agents play $\mathrm{C}$ or $\mathrm{D}$ as long as they "win", i.e. obtain $R$ or $T$ in the PD game, and shift to the other strategy as soon as they "lose" (obtaining $S$ or $P$ ) (Nowak and Sigmund, 1993). In contrast to tit-for-tat, this strategy does not break down in the presence of noise, but can compensate for irrational decisions or mistakes.

As a further mechanism that is mostly relevant for humans, indirect reciprocity captures the idea that "I help you because you helped somebody else before." It thus relies on the reputation of agents. Cooperating with an agent is relatively safe if the agent has a reputation of cooperating often. Conversely, an agent deciding whether to cooperate may take into account the consequences for its reputation. A good reputation will be rewarded by others by choosing to cooperate. In turn, cooperating achieves a good reputation. Hence, indirect reciprocity can promote cooperation among social agents (Nowak and Sigmund, 1998, 2005).

More complex interactions may also help cooperation to evolve. For instance, if the agents can choose to punish defecting interaction partners and/or reward cooperating ones, cooperation may thrive (Sigmund et al. 2001: Sigmund, 2007). Furthermore, dropping the requirement for agents to always participate in the game has a similar effect. If the agents can volunteer for playing the game, still obtaining a fixed baseline payoff if they do not participate, cooperation does not die out (Hauert et al. 2002).

In the example in 6.2.2. we found that cooperation cannot survive under $\mathrm{PD}$ dynamics in a well-mixed population, in which everybody interacts with everybody else with the same probability. But the mixing assumption is a strong over-simplification and does not hold in most real-world populations. Agents are more likely to interact if they are spatially close than far apart. We interact more often with friends and colleagues than with strangers. Hence, real-world populations are not well-mixed but structured by spatial and social relations. The resulting heterogeneity in the interaction patterns among the agents has a strong impact on the evolution of cooperation. The influence of this interaction structure can be studied in evolutionary games on lattices and complex networks, in which the agents are represented by the nodes of the network and interact with their direct neighbours only. Thus, the agents collect payoffs from the local interactions on the network, which define their fitness in the local competition with their neighbours.

The competition among the agents in the network can be modelled by many different evolutionary rules defining the transformation rules and the update scheme for the 


\section{Evolution of cooperation}

specific model. Popular choices are the so-called birth-death rule, the death-birth rule, the imitation rule, and pairwise comparison (Ohtsuki and Nowak, 2006, Traulsen et al. 2006). According to the birth-death rule, an agent is randomly selected from the population with a probability proportional to its fitness and reproduces to a random neighbouring site, i.e. its offspring replaces a random neighbour. Following the death-birth rule, a random agent is removed from the population, leaving its neighbours to compete for the empty site according to their fitness. Under the imitation rule, a random agent is selected to imitate the strategy of one of its neighbours according to their fitness. Finally, under the pairwise comparison rule, an agent and one of its neighbours are chosen at random using node or link update (cf. 3.1.2) and one of them imitates the other with a probability proportional to their fitness difference.

For evolutionary games on networks, it has been reported that the results of specific models depend strongly on the model details, such as the particular choice of one of these update rules (Szabó and Fáth, 2007, Roca et al., 2009). One reason for this is the strong degree bias associated with the different node update rules, as discussed in 3.1.2 Nevertheless, a rather robust result is that cooperation can prevail in "clusters" of cooperators, in which cooperators interact more often with other cooperators than defectors (Nowak and May 1992 . Szabó and Töke, 1998, Hauert, 2002, Doebeli and Hauert 2005). This effect is called network reciprocity.

For degree-regular networks, in which every agent has exactly $k$ neighbours, the relation $b / c>k$ is a necessary condition for cooperators to survive under imitation dynamics (Ohtsuki et al. 2006). In degree-heterogeneous networks, this condition generalizes to $b / c\rangle\left\langle k_{n n}\right\rangle$, where $\left\langle k_{n n}\right\rangle$ is the average nearest neighbour degree in the network (Konno, 2011). Moreover, a modified replicator equation similar to 6.4) can be derived which takes into account the network structure to describe the evolution of cooperation on regular networks (Ohtsuki and Nowak, 2006).

In real social networks, agents establish new contacts or discard existing ones as they encounter other agents either at random or strategically in order to improve their situation. Interaction networks or social networks therefore do not merely play the role of a fixed substrate for the game dynamics, but they are continuously subject to change. Moreover, they may respond adaptively to the agents' behaviour. Thus, the evolution of cooperation in social networks should be modelled using adaptive networks. In the remaining part of this chapter, I discuss some of the main results of recent adaptive-network models of cooperation and present a new model, in which the adaptivity of the network opens a route to asymptotic full cooperation.

\subsection{Cooperation in adaptive networks}

Network reciprocity is an example of how cooperation can be promoted by an appropriate interaction topology. Such an interaction topology may be achieved dynamically if the agents can choose their interaction partners according to their strategies, that is if the 
agents interact in an adaptive network. Moreover, network adaptivity can lead to a number of less obvious mechanisms allowing for the evolution and persistence of cooperation (a list of various co-evolutionary mechanisms including network adaptivity has been assembled by Perc and Szolnoki, 2010). In this section, I give an overview on several different mechanisms by which cooperation can be promoted in adaptive networks.

\section{Local effects}

In adaptive networks, a node is not constrained to remain within the static interaction topology of its-possibly unfavourable-neighbourhood. It can rather try to increase its fitness, which is determined by the payoff it extracts from this neighbourhood, by discontinuing unprofitable links and seeking to establish new advantageous connections, for instance.

It is generally advantageous for cooperators to connect to other cooperators and form tightly connected clusters, as these produce high payoff and cannot be invaded easily by defectors (network reciprocity). In adaptive networks, cooperator clusters can be created actively by appropriate link dynamics directly or indirectly favouring assortative interactions between cooperating agents. Indeed, cooperation prevails when individuals adjust their social ties,' i.e. prefer links to cooperators over links to defectors (Santos et al. 2006a). Such link dynamics can be quite counter-intuitive. In (Zimmermann et al. 2000, 2001. 2004 Eguíluz et al. 2005), for instance, cooperators cannot alter their connections, but defectors are allowed to rewire, seeking new cooperators to exploit. Nevertheless, the population reaches a highly cooperative stationary state that cannot be invaded by defectors.

\section{Structure}

Not only local clustering of cooperators but also heterogeneity in the number of contacts per agent can significantly promote cooperation. Notably, the positive effect of degree heterogeneity has been observed in a somewhat unifying way for different games on scale-free networks (Santos and Pacheco, 2005, Santos et al. 2006b). Santos and Pacheco (2005) argued that cooperators typically occupy the high-degree hub nodes in static scale-free networks, acting as leaders in the population.

In adaptive networks, such leadership emerges naturally in the hierarchical topologies that can self-organize due to the interplay between the strategy dynamics and the topological dynamics. Starting from some initial configuration, the network may thus be reshaped into a more favourable structure enabling cooperators to survive. This was first observed in (Zimmermann et al. 2000, 2001, 2004, Eguíluz et al. 2005), where an initially random network evolves into a stationary hierarchy of well-connected influential cooperators (leaders), which are imitated by a large number of followers. Consequently, a substantial fraction of the population cooperates.

Similarly, broad-scale topologies have been observed to self-organize in a different 


\section{Evolution of cooperation}

model by Ebel and Bornholdt (2002), which is based on an iterated version of the $\mathrm{PD}$ game. There, in addition to promoting cooperation, the topological dynamics also lead to increased clustering and assortative mixing, features observed in many real-world networks.

\section{Mixing}

Although the topological change in adaptive networks may lead to a high level of heterogeneity, it can also reduce the influence of the network structure on the node dynamics by providing an effective mixing of the population. When the agents are allowed to choose their connections in a sufficiently random way, they effectively sample interactions from the whole network. If this proceeds on a fast time-scale, one expects similar dynamics as in well-mixed populations.

When the linking dynamics proceed totally random, the topological evolution effectively decouples from the strategy dynamics, simply mixing the underlying network structure. In this limit, the network is not an adaptive network any more. This was observed, for instance, in a $\mathrm{PD}$ model with random, state-independent rewiring of the adjacent links after each node update (Kun and Scheuring, 2009). Due to this effective mixing, the probability for cooperators to survive and spread is very low, similar to the case of a well-mixed population.

Active linking, on the other hand, may result in a rather different behaviour. In this case, links are formed and disconnected at state-dependent rates. If these processes are fast compared to the node updates, the situation is transformed into an effective game in a well-mixed population with a rescaled payoff matrix, which depends on the stationary regime of the linking processes (Santos et al. 2006b; Pacheco et al. 2006a b). The $\mathrm{PD}$ game can thus be mapped on an effective coordination game in a well-mixed population, where cooperation is greatly enhanced. Similarly, the $\mathrm{SD}$ game is transformed into an effective harmony game, which even allows for full cooperation of every single agent. The number of cooperators surviving in the stationary state is thus a function of the ratio between the time scales of the strategy dynamics and active linking.

\section{Growth}

So far, we have considered only adaptive networks of a fixed size, i.e. a given number of agents. The complex dynamics during network formation and growth, however, also play an important role for the evolution of cooperation. When the interaction network grows adaptively, i.e. new agents connect to existing ones depending on the strategy dynamics, cooperation can be favoured by a novel mechanism rooted in this adaptive growth process.

Two prominent examples are the growing network models of Ren et al. (2006) and Poncela et al. (2009b). In these models, new agents are added continuously and connect to existing ones according to a preferential attachment rule. In contrast to the $\mathrm{BA}$ algorithm 
for scale-free networks, here the preference is for fitness rather than degree. Thus, the agents receive new connections with a probability proportional to their fitness. With this rule, highly hierarchical networks emerge where the most successful players in terms of payoff are also the most connected ones. For the imitation processes, a probabilistic update rule is used in synchronous updates of all agents, so that they occasionally adopt the worse strategy. Notably, a high level of cooperation is achieved in (Poncela et al. 2009b), which decays once the network stops growing. Hence, a genuinely novel mechanism is at work in this model: It is the preferential growth process itself that facilitates cooperation in this system rather than the resulting heterogeneous hierarchical topology.

In a stricter variant of the model where the agents never adopt a worse strategy, cooperation increases even further after the final system size has been reached (Poncela et al. 2008). Thus, only rather rational agents (never adopting a worse strategy) can achieve and sustain a cooperative state when the network stops growing. Still, the mechanism at work is different from the way cooperation is promoted in static scale-free networks (Santos and Pacheco, 2005). In contrast to the latter, cooperators do not necessarily occupy the most connected hub nodes. In fact, a high level of cooperation is reached 'despite the presence of defector hubs' (Poncela et al. 2009a).

\section{Dynamics}

The central question in evolutionary game theory is how cooperation can evolve and survive in a population of selfish individuals. For this reason, most studies in this field focus on average and therefore quasi-static quantities such as the stationary fraction of cooperators in the population or the probability that an initially rare strategy can spread and fixate in the population (fixation probability). Cooperation on adaptive networks is, however, an inherently dynamic process exhibiting a number of interesting phenomena apart from the fact that it can be promoted substantially by network adaptivity. We have already seen that cooperation may be promoted dynamically as long as the network keeps growing. Furthermore, oscillations in the number of cooperators have been observed in adaptive networks (Hanaki et al. 2007, Suzuki et al. 2008: Szolnoki et al. 2008: Szolnoki and Perc, 2009a b). In (Zimmermann et al. 2000, 2001, 2004:Zimmermann and Eguíluz, 2005. Eguíluz et al. 2005), a perturbation of the highly cooperative stationary state results in large reorganization avalanches until a new stationary regime of high cooperation is reached.

In the remaining part of this chapter, I discuss how cooperation can be promoted dynamically in a specific model where the agents have access to non-local information about the average performance of the strategies. In this model, fast topological change can even achieve asymptotic full cooperation in infinite populations. 


\subsection{A homoclinic route to full cooperation ${ }^{6}$}

As already pointed out, cooperation on adaptive networks is an inherently dynamical process. Still, the role of dynamical phenomena in these systems has not received much attention, as the research traditionally focuses on the stationary (average) level of cooperation or the fixation probability of a strategy invading a homogeneous population, which are both quasi-static quantities.

Dynamical phenomena such as the appearance of oscillations are known, however, to play a role in non-adaptive models of iterated and cyclic games, where they have been investigated thoroughly (e.g., Nowak and Sigmund, 1989. Imhof et al. 2005, Reichenbach et al. 2006). Only recently, oscillations have also been observed in an adaptive-network model, where they were interpreted in terms of a Red Queen mechanism that is also able to promote cooperation (Szolnoki and Perc, 2009b).

In the following, we study an evolutionary game on an adaptive network where the collective dynamics of the agents lead to asymptotic full cooperation. In contrast to previous work that mainly focused on local update rules based on the payoff of neighbouring nodes, the agents use non-local information about the general performance of the strategies in our model. This corresponds to the accessibility of certain global knowledge in social systems through, e.g., the media.

We find oscillations in the number of cooperating players, in which the time-averaged payoff of the latter equals that of the defectors in the limit of infinite population size. Nevertheless, a state of full cooperation is approached asymptotically if the rate of topological change exceeds a finite threshold. This can be understood from the emergent-level description of the model in terms of a low-dimensional ODE system. The analysis of this ODE system reveals the formation of a homoclinic loop in a global bifurcation, which is interpreted as the underlying mechanism for the asymptotic approach to full cooperation.

We show that in finite populations, this mechanism can lead to periods of almost full cooperation interrupted by recurrent collapses to episodes of predominant defection, revealing a possible scenario for the sudden failure of cooperation in real systems (Chu and Lee, 1994, Turchin, 2003).

\subsubsection{Model}

We consider an undirected network of $N$ nodes, representing agents, and $K$ links, representing interactions. Each agent $i$ is assigned a strategy $\sigma_{i}$ from the strategy set $\mathcal{S}=\{\mathrm{C}, \mathrm{D}\}$, which can either be cooperation, $C:=1$, or defection, $\mathrm{D}:=2$. The interactions among the agents are modelled by the $\mathrm{SD}$ game (cf. 6.2.2) using the benefit-cost payoff matrix (6.5),

$$
\mathbf{M}=\left(\begin{array}{cc}
b-\frac{c}{2} & b-c \\
b & 0
\end{array}\right)
$$

\footnotetext{
${ }^{6}$ This section is based on the author's article (Zschaler et al. 2010) published in the New Journal of Physics.
} 
The payoff a player $i$ receives from the interaction with player $j$ can then be written as $M_{\sigma_{i} \sigma_{j}}$. The total payoff player $i$ gains from all interactions is given by $\pi_{i}=\sum_{j \sim i} M_{\sigma_{i} \sigma_{j}}$, where the summation runs over all $j$ linked to $i$.

Starting from a random graph and randomly assigned equiprobable strategies, we evolve the network in a similar way as for the VM in chapter 5 . In every time step, one link is selected at random (link update). With probability $p$, this focal link is rewired. Otherwise, i.e. with probability $q=1-p$, one of the linked players adopts the other player's strategy. For large $p$, the players thus tend to change their interaction partners, whereas for small $p$ they tend to revise their behaviour.

To complete the model, we have to specify which agent copies the other's strategy in a strategy adoption event, and which agent keeps the link when rewiring takes place. Most previous models assume that the agents' access to information is governed by the same network as the underlying games, forcing the agents to base their decisions on information from direct neighbours. The same network topology thus determines three different aspects of the system: the interaction partners of an agent, against whom the game is played, the potential role models, whose strategies can be adopted, and the agents from which information can be obtained. However, for intelligent agents, and especially humans, there is no reason to assume that these three network roles are all fulfilled by coinciding topologies (Ohtsuki et al. 2007). In fact, the use of identical networks for information and state transmission has recently been criticized for the related application of epidemic spreading (Funk et al. 2009, 2010).

Here, we assume that information transfer in the population is not governed exclusively by the interaction network. As a first approximation, we consider the simplest case in which the information transmission network is replaced by an effective global coupling, as information can be rapidly transmitted and is also transported by the mass media in the human population. In the main part of this work, we assume that the agents rely on their perception of a strategy's general performance. This global measure for a strategy $\sigma$ is obtained as the average payoff of all agents currently using $\sigma$,

$$
\phi(\sigma)=\sum_{\sigma_{i}=\sigma} \frac{\pi_{i}}{[\sigma] N}=\sum_{\sigma^{\prime} \in \mathcal{S}}\left(1+\delta_{\sigma \sigma^{\prime}}\right) M_{\sigma \sigma^{\prime}} \frac{\left[\sigma \sigma^{\prime}\right]}{[\sigma]}
$$

where $\delta$ is the Kronecker delta, $[\sigma]$ is the fraction of the population using the strategy, and $\left[\sigma \sigma^{\prime}\right]$ is the number of links between agents using strategies $\sigma$ and $\sigma^{\prime}$ normalized by $N$. The validity of assuming such a measure based on global information is discussed in section 6.5.3. where we consider the effect of a finite "information horizon" within which the agents assess the performance of a strategy.

If strategy adoption occurs on a link connecting the agents $i$ and $j$, then we assign the strategy of agent $i$ to agent $j$ with probability given by the Fermi function (Blume. 1993. Szabó and Tőke, 1998)

$$
f_{\beta}(i, j)=\left(1+e^{-\beta\left[\phi\left(\sigma_{i}\right)-\phi\left(\sigma_{j}\right)\right]}\right)^{-1} .
$$



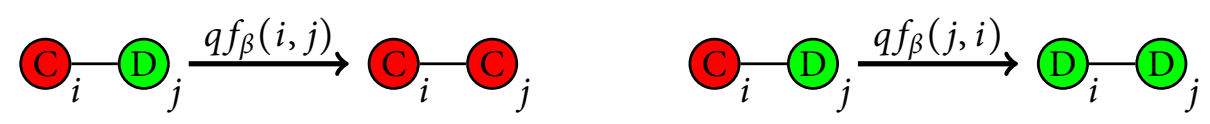

Figure 6.2.: Imitation rules. Whether node $i$ imitates $j$ or vice versa depends on the non-linear rate function $f_{\beta}(i, j)$, which involves the general performances of the strategies as perceived by the agents.
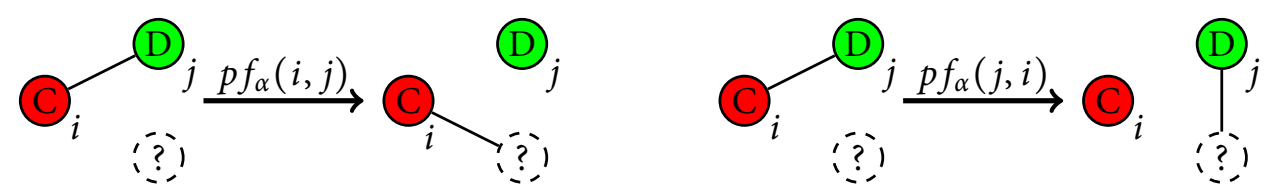

Figure 6.3.: Rewiring rules. Whether node $i$ or $j$ keeps the link in a rewiring event depends on the non-linear rate function $f_{\alpha}(i, j)$. A dashed circle represents a node picked at random from the whole population.

Otherwise, i.e. with probability $f_{\beta}(j, i)=1-f_{\beta}(i, j)$, the strategy of agent $j$ is assigned to agent $i$ (Fig.6.2).

The parameter $\beta$ is the selection intensity and corresponds to an inverse temperature. For small $\beta$, the strategy adoption is almost random, whereas for $\beta \rightarrow \infty$, the more successful strategy is always adopted. Following established practice (Nowak et al., 2004, Ohtsuki et al. 2006, 2007), we mainly focus on the case of weak selection (small $\beta$ ), which is known to be highly relevant for biology and supported by recent evidence for social systems (Traulsen et al. 2010). Weak selection also implies that an agent's local neighbourhood is more important for an agent's strategy than the global information. While the global information (weakly) influences the probability that the agent's strategy changes in a given strategy update, the local neighbourhood governs the rates at which update events involving the focal agent occur.

In a similar fashion, we assume that the players using the more successful strategy are more likely to keep links during rewiring events. If a rewiring event occurs on a link connecting the agents $i$ and $j$, the link is cut and then a new link is established between a randomly selected agent $k$ and agent $i$ (with probability $f_{\alpha}(i, j)$ ) or between $k$ and $j$ (with probability $f_{\alpha}(j, i)$ ) (Fig.6.3). Here, we have used the Fermi function with selection intensity $\alpha$ to capture that agents following a successful strategy may find it easier to attract new contacts.

Using the average payoff $\phi(\sigma)$ in rewiring instead of the payoff obtained by individual agents prevents successful players from acquiring an unrealistically large number of links, which could otherwise lead to the formation of star-like topologies. It also implies that all agents using the same strategy are considered equivalent for the dynamics. We note that a similar assumption is made in the large class of models where the topological processes depend exclusively on the nodes' states instead of their fitness (e.g. Pacheco et al. 2006a: van Segbroeck et al. 2009). 

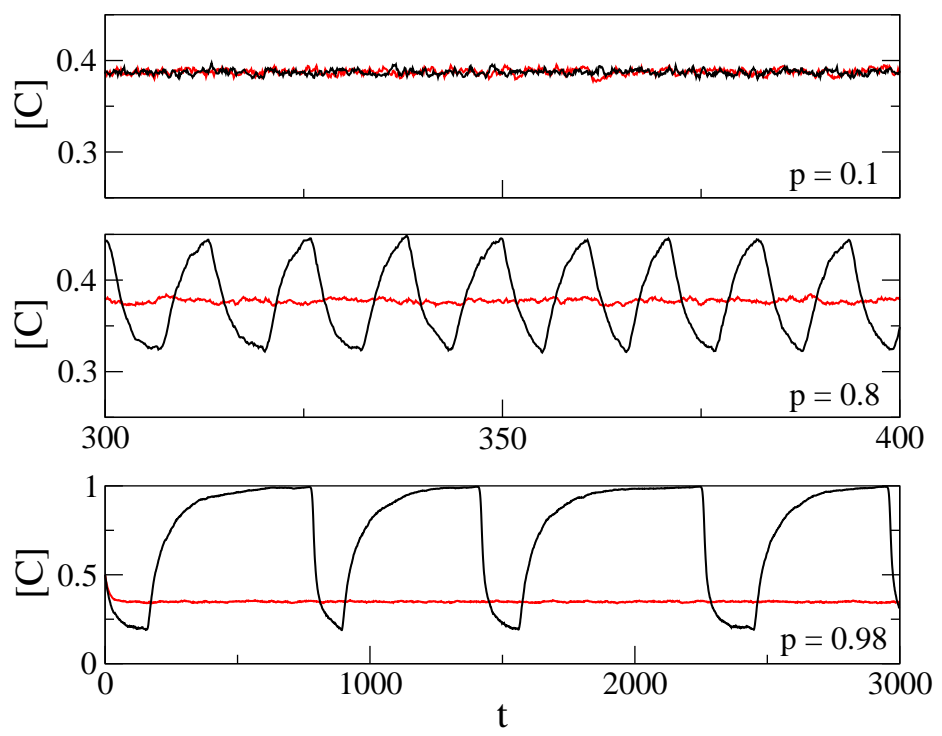

Figure 6.4.: Time series of the fraction of cooperators in an adaptive network for different rewiring rates $p$. When rewiring occurs almost at random $(\alpha=0.1$, red), cooperation and defection coexist at a stationary level for all $p$. When players following the more successful strategy keep the link with high probability ( $\alpha=30$, black), oscillations appear as the rate of rewiring exceeds a critical threshold. Parameters: $N=10^{5}, K=10^{6}, \beta=0.1, b=1, c=0.8$.

\subsubsection{Simulation results}

In order to explore the dynamics of the model, we run individual-based stochastic simulations for $N=10^{5}$ and $K=10^{6}$. Typical time series for different rewiring rates $p$ are shown in Fig. 6.4 For weak selection $(\alpha, \beta \ll 1)$, the system approaches a stable steady state where both strategies coexist. In this regime the stationary density of cooperators depends only weakly on $p$. If rewiring is strongly selective $(\alpha \gg \beta)$, then the dynamics depend strongly on $p$. Stationary behaviour is still observed if $p$ is small, but as $p$ increases, the system undergoes a continuous transition in which the density of cooperators starts to oscillate. The same transition can also be observed for higher $\beta$, but is shifted to greater values of $p$. As $p$ is increased further, the amplitude and period of the cycle grows. At higher $p$, long periods of almost full cooperation appear, which are interrupted by sudden episodes of defection.

To understand the onset of oscillations, note first that in the stationary state the average payoffs of cooperators and defectors have to be identical, so that strategy adoption and rewiring happen randomly. If, due to fluctuations, cooperators receive a slightly higher payoff than defectors, then the density of C-C links, [CC], starts to increase due to the effect of the strongly selective rewiring, which tends to de-mix the network by accumulating links in the population with the higher payoff. The increasing [CC] constitutes a positive feedback increasing the payoff of the cooperators further. As agents adopt 


\section{Evolution of cooperation}

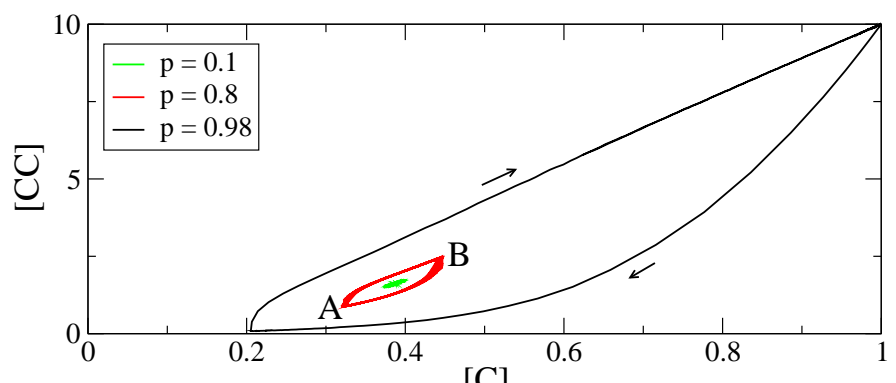

[C]
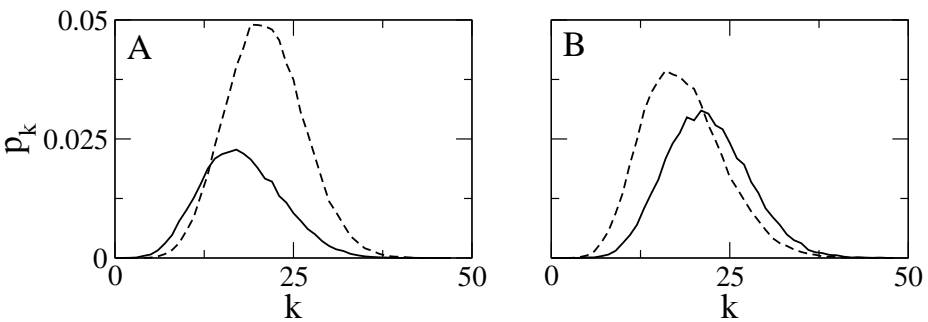

Figure 6.5.: Cycles of cooperation and defection in agent-based simulations. Top: dynamics in the [C]-[CC]-plane for rewiring selection intensity $\alpha=30$. Bottom: degree distributions of cooperators (solid) and defectors (dashed) for rewiring rate $p=0.8$ at the two turning points of the cycle. Simulation parameters as in Fig. 6.4

the cooperating strategy, the system approaches a state where both [C] and [CC] are high (point B in Fig. 6.5). In this state, strategy adoption can overcome the de-mixing effect of rewiring because adoption of the defecting strategy by cooperators creates many C-D-links. The payoff of defectors rises rapidly, leading the system back to a mixed state, where a substantial number of agents are defecting.

\subsubsection{Analytical results}

To gain a deeper understanding we formulate a low-dimensional, emergent-level description of the present model according to the moment-expansion procedure in section 3.3 . Closing the obtained ODE system at the mean-field level yields a one-dimensional ODE for the density of cooperators, [C], which cannot reproduce oscillatory long-term dynamics. We therefore close the system at the level of links using again the $\mathrm{PA}$.

We treat $[C],[C C]$, and $[D D]$ as dynamical variables, whereas $[D]$ and $[C D]$ are given 
by the conservation laws $[\mathrm{C}]+[\mathrm{D}]=1$ and $[\mathrm{CC}]+[\mathrm{DD}]+[\mathrm{CD}]=\langle k\rangle / 2$. Thus we obtain

$$
\begin{aligned}
\frac{\mathrm{d}}{\mathrm{dt}}[\mathrm{C}] & =q[\mathrm{CD}]\left(f_{\beta}-\bar{f}_{\beta}\right), \\
\frac{\mathrm{d}}{\mathrm{dt}}[\mathrm{CC}] & =p[\mathrm{C}][\mathrm{CD}] f_{\alpha}-p[\mathrm{D}][\mathrm{CC}]+q[\mathrm{CD}]\left\{\left(1+\frac{[\mathrm{CD}]}{[\mathrm{D}]}\right) f_{\beta}-2 \frac{[\mathrm{CC}]}{[\mathrm{C}]} \bar{f}_{\beta}\right\}, \\
\frac{\mathrm{d}}{\mathrm{dt}}[\mathrm{DD}] & =p[\mathrm{D}][\mathrm{CD}] \bar{f}_{\alpha}-p[\mathrm{C}][\mathrm{DD}]+q[\mathrm{CD}]\left\{\left(1+\frac{[\mathrm{CD}]}{[\mathrm{C}]}\right) \bar{f}_{\beta}-2 \frac{[\mathrm{DD}]}{[\mathrm{D}]} f_{\beta}\right\},
\end{aligned}
$$

where we have introduced the abbreviated notation $f_{\xi}=1 /\left(1+e^{-\xi[\phi(C)-\phi(D)]}\right)$ and $\bar{f}_{\xi}=1-f_{\xi}$. In (6.9), the first factor, $q[\mathrm{CD}]$, denotes the rate of strategy adoption events, while the second factor is the expected change in [C] in each such event. Analogously, the first two terms in 6.10) and (6.11) describe the gain and loss rates of the respective link density due to rewiring, while the third and fourth terms account for the link creation and loss due to strategy adoption.

In the regime of weak rewiring selection, $\alpha \ll 1$, and weak strategy selection, $\beta \ll 1$, our model reduces to previously studied systems in two important limiting cases. For fast rewiring $(p \approx 1)$, which is almost random when $\alpha$ is small, the system evolves according to standard replicator dynamics in a well-mixed population (cf. 6.2.2). In this case, the mean-field approximation of equation (6.9) reduces to

$$
\frac{\mathrm{d}}{\mathrm{dt}}[\mathrm{C}]=\frac{q\langle k\rangle}{2}[\mathrm{C}][\mathrm{D}](\phi(C)-\phi(D))=\frac{q\langle k\rangle}{2}[\mathrm{C}](\phi(C)-\bar{\phi}),
$$

which is, up to a time scale, the replicator equation $(6.3)$. On the other hand, when strategy adoption is much faster than rewiring, $p \ll 1$, the network is almost static and the modified replicator equation for static regular graphs may be used as an approximation (Ohtsuki and Nowak, 2006).

We now use the low-dimensional model, (6.9)-6.11), to explore the system with the tools of non-linear dynamics. The bifurcation diagram of the ODE system in Fig. 6.6 is in good agreement with the results of the agent-based simulations. If rewiring is slow (small $p$ ), the system approaches an equilibrium in which cooperators and defectors coexist. For strongly selective rewiring $(\alpha \gg \beta)$, however, a critical threshold $p$ exists at which this steady state is destabilized in a supercritical Hopf bifurcation (point $S$ in Fig. 6.6) and a stable limit cycle emerges, explaining the onset of oscillations (Fig. B.4). The now unstable stationary state still exists for larger $p$, converging to the mean-field value determined by the replicator equation (6.12) for $p \rightarrow 1$.

As $p$ is increased further, the amplitude of the limit cycle grows. Eventually, the cycle undergoes a homoclinic bifurcation as its upper turning point connects to the fully cooperative state $([\mathrm{C}]=1,[\mathrm{CC}]=\langle k\rangle / 2,[\mathrm{DD}]=0)$. Dynamically, this state is a saddle point, which the cycle approaches along its stable manifold and leaves along the unstable manifold (Guckenheimer and Holmes, 2002), forming a homoclinic loop as shown schematically in Fig. B.5 In the saddle point, the velocity at which the system 


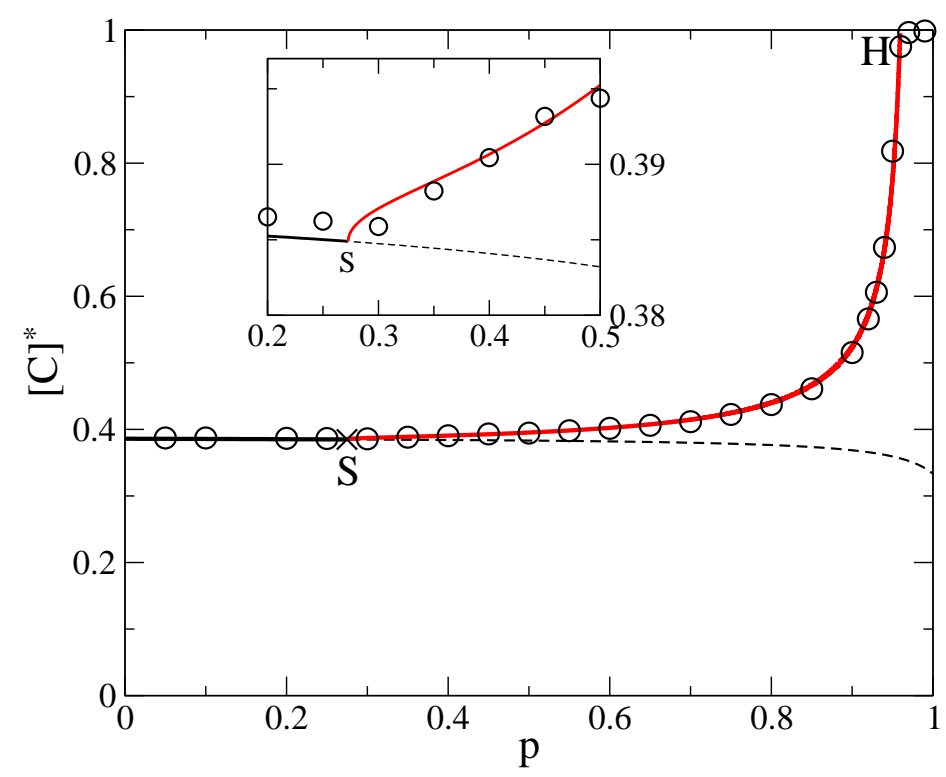

Figure 6.6.: Bifurcation diagram for the case of strong rewiring selection $(\alpha=30)$. If rewiring is slow (small $p$ ), cooperators and defectors coexist in a stable steady state. At higher rewiring rates the stability is lost in a supercritical Hopf bifurcation (S), from which a stable limit cycle emerges. The limit cycle undergoes a homoclinic bifurcation in point $\mathrm{H}$ as it connects to a saddle point at $[\mathrm{C}]=1$. The lines show the stable (solid) and unstable (dashed) steady state, and the upper turning point of the limit cycle (red), computed in the low-dimensional model. Circles denote agent-based simulation results for $N=10^{5}, K=10^{6}$. The inset shows a blow-up of the bifurcation point S. See Fig. 6.4 for parameters.

moves along the cycle approaches zero. If observed at a random point in time, the ODE system is therefore found to be in the fully cooperative state with probability one.

Let us emphasize that the asymptotic full cooperation is a purely dynamical effect. The existence of a limit cycle shows that the time-averaged fitnesses of cooperators and defectors are equal. However, in the homoclinic bifurcation the time-average becomes meaningless as it has to be taken over infinite time, while for any finite time the cooperators dominate.

The deterministic description provided by the ODE system holds in the thermodynamic limit of large system size. In the agent-based model, full cooperation is an absorbing state of the strategy dynamics. Small systems can reach this state and remain at full cooperation. In larger systems $(N>100)$, the system slowly approaches the saddle point along the stable manifold, but is eventually carried over to the unstable manifold by fluctuations. Once on the unstable manifold, defection rapidly invades the population, launching the system into another round on the cycle before the fully cooperative state is approached again (Fig. 6.5). Because of their stochastic excitable nature, these invasions of defectors occur at irregular time intervals (Fig. 6.4 bottom), becoming longer with 

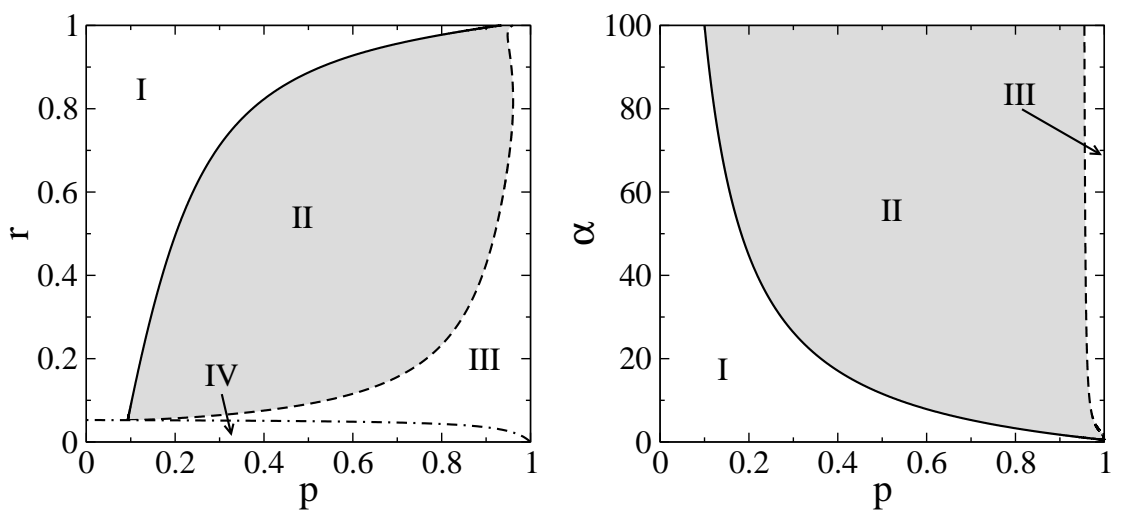

Figure 6.7.: Two-parameter bifurcation diagrams showing the dependence on the rewiring rate $p$ and the cost-to-benefit ratio (Doebeli and Hauert 2005) $r=c /(2 b-c)$ (left, for $\alpha=30$ ), and the rewiring selection strength $\alpha$ (right, for $b=1, c=0.8$ ), resp. In region I, cooperation and defection coexist in a stationary state. A Hopf bifurcation line (solid) marks the transition to the oscillatory parameter region (II, shaded), which is bounded by a line of homoclinic bifurcations (dashed) leading to asymptotic full cooperation (III). Stable full cooperation (IV) is reached via a transcritical bifurcation (dash-dotted) if $r$ is low. All bifurcation lines meet in a codimension-2 Takens-Bogdanov bifurcation (Guckenheimer and Holmes, 2002). See Fig. 6.6 for additional parameters.

increasing system size.

In the two-parameter bifurcation diagrams shown in Fig. 6.7, the Hopf and homoclinic bifurcation points form lines, which separate parameter regions of qualitatively different long-term dynamics. The oscillatory dynamics and asymptotic full cooperation can be observed in a large parameter region, demonstrating the robustness of the observed phenomenon to the SD game conditions (parametrized by the cost-to-benefit ratio $r=$ $c /(2 b-c)$ of mutual cooperation) and the rewiring selection strength $\alpha$. For larger $\alpha$, the Hopf bifurcation occurs already at slower rewiring rates (Fig. 6.7 right), whereas the opposite is true for larger $\beta$ (not shown): Although stronger replacement selection leads to oscillations at higher frequencies than before, it can be balanced by sufficiently fast rewiring and does not necessarily counteract the homoclinic mechanism.

Finally, we ask how our findings depend on our initial assumption that the agents have access to global, i.e. population-wide information in terms of $\phi(\sigma)$. To that end, we let the players estimate the fitness of strategies by averaging over a neighbourhood of nodes they can reach in a finite number of steps rather than over the whole population. In this case, the transition rates associated to the rules in Fig. 6.2 and Fig. 6.3 do not only depend on the global averages $\phi(C)$ and $\phi(D)$, but on the corresponding averages in a local neighbourhood. Because of their non-linearity due to the presence of the Fermi function, however, it is no longer possible to average the transition rates over the whole population in order to derive the emergent-level $\mathrm{ODE}$ description. Further, it is also not possible to 


\section{Evolution of cooperation}

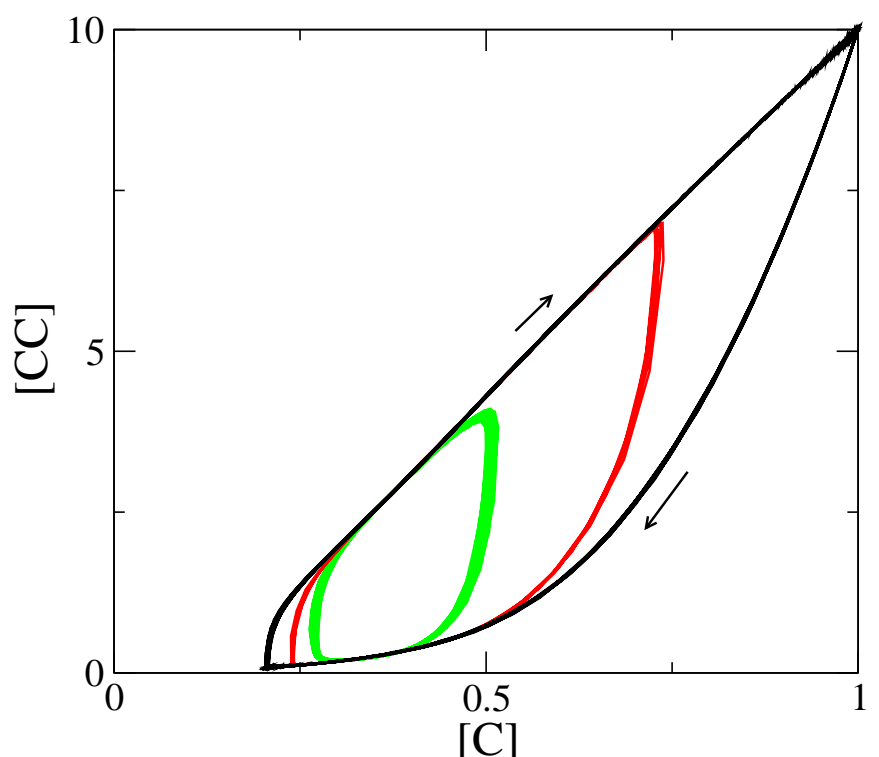

Figure 6.8.: Effect of increasing the agents' information horizon for fast rewiring in simulations. When the agents assess a strategy's fitness by averaging over the accumulated payoffs of the focal link's nearest neighbours only (green), a small limit cycle is obtained. It is larger when also the next-nearest neighbours are included (red). The size of the cycle grows with increasing averaging radius. The quasi-homoclinic cycle of long cooperative periods interrupted by short bursts of defection is recovered when the average is taken over the whole population (black). Parameters as in Fig. $6.4 p=0.98$.

translate the non-linear transformation rules into additional, linear transformation rules as suggested in 3.3.1. because the transition rates do not simply depend on the numbers of, say, neighbouring cooperators, but rather the payoffs of all players in the neighbourhood. Therefore, we analyse the effect of averaging over a finite local neighbourhood by means of computer simulations.

We observe similar behaviour as before in simulations with such an "information horizon". In particular, fast selective rewiring leads to oscillations whenever this kind of neighbourhood information is used. As can be seen in Fig. 6.8, the amplitude and period of these oscillations grow with the information horizon (for fixed $p$ ). Therefore, access to the information from a sufficiently large neighbourhood is, in the present model, necessary to observe the homoclinic route to full cooperation.

\subsubsection{Conclusions}

In this section, we proposed a model of cooperation on an adaptive network in which agents have access to non-local information. We observed that full cooperation is reached asymptotically through a global dynamical mechanism operating far from equilibrium, 
which is made possible by the interplay between topological and local dynamics in the adaptive network. In addition to the results shown, we have verified that similar dynamics can be observed in other parameter regimes, such as stronger strategy selection, and in variants of the model.

In our model, asymptotic full cooperation is achieved dynamically, rather than by assembling characteristic topologies that allow cooperators to thrive. In finite populations, spontaneous collapses of highly cooperative states are observed. The homoclinic mechanism in combination with noise may thus be a relevant ingredient in the systemic failure of cooperation that is found in real world systems.

Although it is conceivable that global information can be accessible in social systems through, e.g., general beliefs, rumours, or the mass media, we note that this assumption is not necessary for the oscillatory behaviour. When the agents are restricted to a finite "information horizon", the present model still exhibits oscillations.

A sufficiently large information horizon is necessary to observe the homoclinic transition. We note, however, that in a different model in which local update rules are assumed and which explicitly enforces realistic limits on the node degrees, a similar transition was found numerically (Szolnoki and Perc, 2009a). We believe that in the present model, the homoclinic mechanism only requires large information horizons because they prevent the formation of unrealistic star-like topologies, which would otherwise stabilize the dynamics.

More work is certainly necessary to explore the role of the homoclinic mechanism in nature. This work will, however, offer the intriguing possibility to identify and understand the dynamical features promoting cooperation in many systems, and may reveal what causes the sudden collapses of cooperative behaviour observed in the human population.

\subsection{Summary}

In this chapter, the effect of adaptive interaction networks on the evolution of cooperation among inherently selfish individuals was discussed in the framework of evolutionary game theory. We have seen that the dynamical self-organization in adaptive networks can give rise to strong behavioural correlations between interacting agents, because the agents can actively discard unfavourable links and seek profitable connections to cooperators instead. Thus, cooperators can form clusters in the network, in which they interact more often with other cooperators than defectors. Such assortative interactions, termed network reciprocity, are known to promote cooperation.

In adaptive networks, particular topologies can thus be assembled that provide an advantage to cooperators and therefore allow them to survive. In addition to that, we demonstrated in a specific model that cooperation can also be promoted by novel mechanisms arising from the adaptivity of the network. In particular, oscillations and asymptotically full cooperation were found under the assumption that the agents were capable of using non-local information about the (average) performance of their strategies. This is a 


\section{Evolution of cooperation}

reasonable assumption for intelligent agents and especially for human society, in which information is disseminated quickly through, e.g., the media and even made globally accessible.

From the emergent-level ODE description of the model, we identified a novel mechanism which leads to asymptotically full cooperation in infinite systems through the formation of a homoclinic loop in phase space. In finite systems, the motion on the homoclinic loop is subject to intrinsic noise, which causes sudden collapses of highly cooperative states. Our analysis showed that the reason for this is the instability of the fully cooperative state, which is the saddle point the homoclinic loop is attached to. Once it is close enough to the saddle point, small fluctuations can drive the system from the stable to the unstable manifold, on which it moves away rapidly from the state of full cooperation. Therefore, long periods of high cooperation are observed while the system is slowly approaching the saddle point, which are then punctuated by sudden break-downs to low cooperation when it is pushed to the unstable manifold by a finite size fluctuation.

In contrast to known mechanisms leading to a stationary state of increased cooperation in networks, cooperation is promoted dynamically in our model. Full cooperation can be achieved asymptotically even though the fully cooperative state is unstable.

This dynamical mechanism is another example of the complex system-level behaviour made possible by the intimate coupling of local and topological dynamics in adaptive networks. In networks of finite size, this coupling gives rise to two intriguing collective phenomena in our model: the self-organization of the network towards an unstable state, in which almost all agents cooperate, and the sudden, self-reinforcing collapses of these phases of cooperation, which are triggered by small fluctuations. 


\section{Conclusions}

In the present thesis, I have studied adaptive-network models of collective phenomena in different systems within a common modelling framework. In this framework, the evolution of an adaptive-network model is specified by a set of microscopic transformation rules and a prescription how these rules are applied to the network. By directly implementing the transformation rules, the model can be conveniently studied in computer simulations. Moreover, I have demonstrated how an approximate description of its emergent, macroscopic behaviour can be derived from the transformation rules in terms of low-dimensional $\mathrm{ODE}$.

In chapter 4 we have proposed a non-spatial model for collective motion in insect swarms that is inspired by models of opinion and consensus formation in social groups. It focuses on the adaptive interaction network among the swarm members and neglects all spatial embedding. This model reproduces qualitatively the results of a recent experiment with desert locusts, i.e. the transition from disordered to ordered collective motion with increasing insect density and the intermittent switching regime in the vicinity of the transition point. Our results suggest that the adaptive nature of the insects' interaction network is an essential requirement for the swarming transition, whereas the spatial embedding and the geometry of the swarm might not be as influential as previously assumed. Moreover, our model allows to identify the minimal microscopic interactions that determine whether the transition to ordered collective motion is continuous or discontinuous. We have seen that a discontinuous transition is only possible if interactions between insects marching in the same direction are included in the model.

In chapter 5 , we expanded on the subject of opinion formation in groups of individuals, moving towards conceptual models for the dynamics of social networks. We have proposed an extension of the adaptive VM to directed networks, which takes the asymmetry of social relationships into account and allows to study different realistic out-degree distributions. The fragmentation transition observed in undirected networks is also present in our model. In addition to that, we have observed early fragmentation in networks where a significant number of nodes with low out-degree can lead to the formation of self-stabilizing structures. Due to the formation of such structures, the network splits into two disconnected components already at comparably low rewiring rates. This new phenomenon relies on the symmetry breaking in the microscopic transformation rules introduced by the link directionality and the build-up of degree correlations.

In chapter 6. we applied our modelling framework to study the evolution of cooperation in groups of self-interested individuals. How cooperative behaviour can emerge and survive in populations of selfish agents is a long-standing question in evolutionary biology, sociology, and economics, which is generally addressed in the frame of evolutionary 


\section{Conclusions}

game theory. We proposed an adaptive-network model based on the paradigmatic SD game, in which cooperation is promoted by a new dynamical mechanism. The coupling of local and topological dynamics in this model gives rise to oscillations during which the system spends more time in states with a high level of cooperation than in states with a lower level. Cooperation is thus promoted dynamically in our model, in contrast to models where high levels of cooperation are achieved in the stationary regime. The approximate emergent-level description of our model revealed that fast rewiring can lead to asymptotic full cooperation in infinite systems via a homoclinic bifurcation. This insight also allows to understand the sudden collapses of highly cooperative states in finite systems, which are triggered by small fluctuations close to the unstable state of full cooperation in the population.

The results presented in this thesis illustrate one of the main advantages of the "network perspective"-it provides an abstraction of complex systems, allowing to draw high-level analogies between seemingly disparate research fields and phenomena. As a particular example, we have used ideas from the research on opinion formation in social networks to develop a new modelling approach for collective motion, which emphasizes the similarities between swarming and consensus formation in social groups. Moreover, our approach has also provided an important basis for subsequent research on the dynamics of fish swarms containing informed and uninformed individuals, in which an extension of our model is considered (Couzin et al. 2011).

The concept of adaptive networks provides a natural way of describing many real-world systems, because it combines the dynamics of the network and the dynamics on the network instead of treating them separately. It contributes to the understanding of collective phenomena in complex systems both because it is a modelling approach complementing traditional approaches (as demonstrated in the swarming model) and because most complex systems can indeed be regarded as having a network structure that changes adaptively over time. In the present work, we were able to study emergent collective phenomena in different complex systems within a common modelling framework by considering these systems as adaptive networks.

We have seen that the feedback loop between local and topological dynamics in adaptive networks can give rise to novel phenomena. In the three proposed models, global order can be achieved by local rules, which is observed as ordered collective motion in the swarming model, as global consensus in the $\mathrm{VM}$, and as asymptotic full cooperation in the cooperation model.

Furthermore, phase transitions between different dynamical regimes involving both local and topological degrees of freedom can occur in adaptive networks. We have interpreted such transitions as bifurcations in the underlying dynamical system. The disorder-order transition in the swarming model, for instance, corresponds to a super- or subcritical pitchfork bifurcation in the emergent-level ODE description. The fragmentation transition in the adaptive $\mathrm{VM}$ can be interpreted as a transcritical bifurcation, in which the connected state loses stability and the fragmented state becomes stable. The observed 
oscillations in the cooperation model are associated with a Hopf bifurcation, and the irregular periods of almost full cooperation in finite systems can be understood from the existence of an infinite-period limit cycle at a homoclinic bifurcation in infinite systems.

The current adaptive-network models necessarily remain mostly conceptual, including the models presented in this work. Nevertheless, they are of twofold use. First, they illustrate the wide range of new phenomena that can be expected from the interplay between local dynamics and topological evolution in adaptive networks. Second, these simple models are an important test bed for the analytical approaches and tools aiming at the new methodological challenges associated with adaptive networks.

To gain an understanding of the dynamical mechanisms behind the observed collective behaviour in our models, we mainly used the moment expansion approach and the $\mathrm{PA}$ to describe them analytically. We have shown that this approach, which was originally developed to study dynamical processes on static networks, is also a useful tool for the analysis of adaptive networks. The moment equations can be conveniently derived from the transformation rules in our modelling framework. By means of the moment expansion, one can capture the global behaviour of the high-dimensional system that is an adaptive network in a low-dimensional system of ODE, which can be analysed using dynamical systems theory.

As the moment closure relies on mean-field assumptions, the moment expansion approach is expected to give inaccurate or wrong predictions in systems with emerging correlations. This is one of its known shortcomings in static networks, in which it typically fails to capture the transient dynamics before an equilibrium configuration is reached. In adaptive networks, however, the ongoing topological change may counteract the build-up of correlations due to the node state dynamics if it results in an effective mixing of the network connections. We have seen that this is indeed the case for the swarming model and the cooperation model, in which the analytical predictions agree nicely with the results of computer simulations. By contrast, the rewiring dynamics may also promote correlations in adaptive networks, so that the moment expansion approach is bound to fail. Specifically, we have seen that the early fragmentation observed in the directed VM cannot be accounted for by the moment equations, although they do provide a qualitative understanding of "ordinary" fragmentation.

To address the difficulties of an analytical approach to adaptive networks with emerging correlations, a detailed understanding of the failure of the moment closure approximations is necessary for these systems. We hope that the techniques presented in this thesis may serve as a basis for future work in developing improved analytical approaches. Despite their shortcomings, the current low-dimensional descriptions allow for an understanding of the basic mechanisms behind the phenomena in many adaptive networks, as demonstrated in this work. To maintain this advantage of tractability, improved analytical approaches will have to remain rather low-dimensional while possibly being less coarse-grained.

While we have focused on relatively simple microscopic transformation rules in the models considered in this work, rules involving more complex LHS subgraphs, for in- 


\section{Conclusions}

stance, are conceivable. Our approach can be applied to such rules in the same way. Computer simulations of more complex rules will be, however, limited by the strongly increasing memory and computation time requirements these rules imply. In deriving the corresponding moment equations, there is no such limitation, although the number of independent equations also grows quickly. Generating these equations from the model specification can, in principle, be automated. However, suitable approximation schemes beyond the $\mathrm{PA}$ and heuristic triplet approximations for the necessary truncation at higherorder moments are presently not available. The refinement and further optimization of our computer simulations and the development of consistent higher-order approximation schemes thus present two promising directions for future research. 


\title{
List of abbreviations
}

\author{
BA Barabási-Albert \\ CP contact process \\ ER Erdős-Rényi \\ LHS left-hand side \\ ODE ordinary differential equation \\ PA pair approximation \\ PD Prisoner's Dilemma \\ RHS right-hand side \\ SD Snowdrift \\ SIS susceptible-infected-susceptible \\ SPP self-propelled particle \\ vM voter model \\ ws Watts-Strogatz
}





\section{A. Surrogate rules for non-linear rates}

Consider the general rule changing the state of a node $i$ at a rate that depends on the node's local neighbourhood $\mathcal{N}_{i}$ according to

$$
\text { (S) } \stackrel{g\left(\mathcal{N}_{i}\right)}{\longrightarrow} \mathrm{I}_{i} \text {. }
$$

Assuming that $g\left(\mathcal{N}_{i}\right)$ can be written as a truncated power series in the number of Ineighbours connected to $i, g\left(\mathcal{N}_{i}\right)=a_{0}+a_{1} n_{i}(\mathrm{I})+a_{2} n_{i}^{2}(\mathrm{I})$, the change in $[\mathrm{S}]$ is given by

$$
\frac{\mathrm{d}}{\mathrm{dt}}[\mathrm{S}]=-\frac{1}{N} \sum_{\sigma_{i}=\mathrm{S}} g\left(\mathcal{N}_{i}\right)=-a_{0}[\mathrm{~S}]-a_{1}[\mathrm{SI}]-\frac{a_{2}}{N} \sum_{\sigma_{i}=\mathrm{S}} n_{i}^{2}(\mathrm{I}) .
$$

The last term is related to the number of I-S-I-triplets in the network divided by $N$, [ISI]. Using the relation

$$
[\mathrm{ISI}]=\frac{1}{N} \sum_{\sigma_{i}=\mathrm{S}}\left(\begin{array}{c}
n_{i}(\mathrm{I}) \\
2
\end{array}\right)=\frac{1}{2 N} \sum_{\sigma_{i}=\mathrm{S}} n_{i}^{2}(\mathrm{I})-\frac{1}{2 N} \sum_{\sigma_{i}=\mathrm{S}} n_{i}(\mathrm{I})
$$

we obtain

$$
\frac{\mathrm{d}}{\mathrm{dt}}[\mathrm{S}]=-\frac{1}{N} \sum_{\sigma_{i}=\mathrm{S}} g\left(\mathcal{N}_{i}\right)=-a_{0}[\mathrm{~S}]-\left(a_{1}+a_{2}\right)[\mathrm{SI}]-2 a_{2}[\mathrm{ISI}]
$$

In the same way, we can transform the equation for, e.g., [SS],

$$
\begin{aligned}
\frac{\mathrm{d}}{\mathrm{dt}}[\mathrm{SS}] & =-\frac{1}{N} \sum_{\sigma_{i}=\mathrm{S}} g\left(\mathcal{N}_{i}\right) n_{i}(\mathrm{~S}) \\
& =-\frac{1}{N} \sum_{\sigma_{i}=\mathrm{S}}\left\{a_{0} n_{i}(\mathrm{~S})+a_{1} n_{i}(\mathrm{I}) n_{i}(\mathrm{~S})+a_{2} n_{i}^{2}(\mathrm{I}) n_{i}(\mathrm{~S})\right\}
\end{aligned}
$$

and obtain

$$
\frac{\mathrm{d}}{\mathrm{dt}}[\mathrm{SS}]=-2 a_{0}[\mathrm{SS}]-\left(a_{1}+a_{2}\right)[\mathrm{SSI}]-2 a_{2}\left[\mathrm{IS}_{\mathrm{S}}^{\mathrm{I}}\right],
$$

where $\left[\mathrm{IS}_{\mathrm{S}}^{\mathrm{I}}\right]$ denotes the number of "stars" with a central S-node that has two I-neighbours and one S-neighbour. Here, we have used that

$$
\left[\mathrm{IS}_{\mathrm{S}}^{\mathrm{I}}\right]=\frac{1}{N} \sum_{\sigma_{i}=\mathrm{S}}\left(\begin{array}{c}
n_{i}(\mathrm{I}) \\
2
\end{array}\right) n_{i}(\mathrm{~S})=\frac{1}{2 N} \sum_{\sigma_{i}=\mathrm{S}}\left\{n_{i}^{2}(\mathrm{I}) n_{i}(\mathrm{~S})-n_{i}(\mathrm{I}) n_{i}(\mathrm{~S})\right\} .
$$




\section{A. Surrogate rules for non-linear rates}

Following the same argument, the equation for [II] reads

$$
\frac{\mathrm{d}}{\mathrm{dt}}[\mathrm{II}]=\left(a_{0}+a_{1}+a_{2}\right)[\mathrm{SI}]+2\left(a_{1}+3 a_{2}\right)[\mathrm{ISI}]+6 a_{2}\left[\mathrm{IS}_{\mathrm{I}}^{\mathrm{I}}\right]
$$

With a suitable closure approximation for the triplet and quadruplet motifs, equations (A.2)-(A.4) describe the average time evolution of the system. We are looking for a surrogate system of transformation rules involving linear rates only whose emergent-level description leads to the same set of differential equations. To that end, consider the rule system

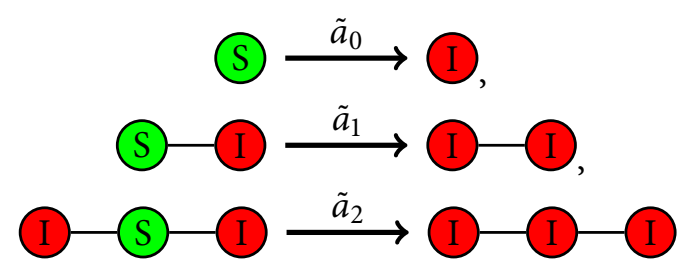

under the direct node update scheme. The corresponding dynamical equations are derived straightforwardly as

$$
\begin{aligned}
\frac{\mathrm{d}}{\mathrm{dt}}[\mathrm{S}] & =-\tilde{a}_{0}[\mathrm{~S}]-\tilde{a}_{1}[\mathrm{SI}]-\tilde{a}_{2}[\mathrm{ISI}], \\
\frac{\mathrm{d}}{\mathrm{dt}}[\mathrm{SS}] & =-2 \tilde{a}_{0}[\mathrm{SS}]-\tilde{a}_{1}[\mathrm{SSI}]-\tilde{a}_{2}\left[\mathrm{SS}_{\mathrm{I}}^{\mathrm{I}}\right], \\
\frac{\mathrm{d}}{\mathrm{dt}}[\mathrm{II}] & =\left(\tilde{a}_{0}+\tilde{a}_{1}\right)[\mathrm{SI}]+2\left(\tilde{a}_{1}+\tilde{a}_{2}\right)[\mathrm{ISI}]+3 \tilde{a}_{2}\left[\mathrm{IS}_{\mathrm{I}}^{\mathrm{I}}\right] .
\end{aligned}
$$

Comparing the coefficients with (A.2)-A.4 yields

$$
\tilde{a}_{0}=a_{0}, \quad \tilde{a}_{1}=a_{1}+a_{2}, \quad \tilde{a}_{2}=2 a_{2} .
$$

This means that the average dynamics of the transformation rule involving the quadratic rate $g\left(\mathcal{N}_{i}\right)$ and the above rules with linear rates according to $(\mathrm{A.8})$ are described by the same ODE model. They are therefore expected to show the same macroscopic behaviour. For the purpose of studying its emergent-level properties, the original non-linear transformation rule can thus be replaced by a set of linear rules involving link and triplet motifs.

The advantage of this surrogate system is twofold. First, replacing the non-linear rates by linear ones makes it possible to formulate an $\mathrm{ODE}$ description that involves only the macroscopic network moments as dynamical variables, although this comes at the cost of introducing higher-order moments. Second, simulating the full network dynamics for linear rules, in which the transition rates are the same for every instance of the LHS motif in the network, is often less computationally costly than simulating with non-linear transition rates, which are essentially different across the network. 
In principle, the surrogate system approach can be also used for transition rates involving greater powers of $n_{i}(\mathrm{I})$. However, more replacement rules involving LHS motifs of even higher order are required in this case, often complicating the construction of the corresponding rate equations. Moreover, the surrogate system is not necessarily advantageous in computer simulations if motifs of very high order are involved. 



\section{B. Bifurcations in dynamical systems}

In this section, I give a brief overview of the basic concepts of dynamical systems theory that are most relevant for the purpose of this work. It is not intended to be mathematically rigorous, but rather a pragmatic glossary of the analytic tools that allow further analysis of the moment equations. For a detailed, mathematically sound introduction to dynamical systems theory, I refer the reader to standard textbooks in this field, as for instance Kuznetsov (1998), Strogatz (2001), or Guckenheimer and Holmes (2002).

The models considered in the present work are defined in terms of microscopic transformation rules, from which a macroscopic, emergent-level description is derived. In general, this macroscopic description is formulated as a $d$-dimensional, deterministic, autonomous $\mathrm{ODE}$

$$
\dot{\mathbf{x}}(t)=\mathbf{f}\left(\mathbf{x}(t), \mu_{1}, \mu_{2}, \ldots, \mu_{m}\right),
$$

where $\mathbf{x}(t) \in \mathbb{R}^{d}$ is the $d$-dimensional moment vector, whose time evolution is determined by the vector field $\mathbf{f}: \mathbb{R}^{d+m} \rightarrow \mathbb{R}^{d}$. This function depends on the parameters $\mu_{1}, \ldots, \mu_{m}$, as for instance the rate constant $p$ in the $\mathrm{CP}$ rule in Fig. 3.1. (a).

The long-term dynamics of an ODE system are determined by the structure of its phase space, which is spanned by all possible system trajectories $\mathbf{x}(t)$, i.e. solutions of (B.1). For the purpose of the present work, two common subsets of an ODE system's phase space are of particular interest: fixed points and limit cycles. Fixed (or stationary) points are points in phase space, for which the right-hand side of (B.1) vanishes. Thus, a trajectory that reaches a fixed point stays there. A limit cycle is an isolated closed trajectory in phase space, on which the system moves periodically. In general, the phase space may be more complex than just containing fixed points and limit cycles. In particular, intricate fractal structures may be present. This is, however, not the case for the models considered in the present work.

Fixed points and limit cycles are said to be Lyapunov stable if all trajectories starting close to them remain close. They are called (asymptotically) stable if all nearby trajectories come even closer in the course of time. Conversely, they are called unstable if there are close trajectories that leave their vicinity.

For a given parameter set $\left\{\mu_{i}\right\}$, fixed points and limit cycles can be computed from the right-hand side of the ODE. It is important to know whether their existence and stability are affected if some of the $\mu_{i}$ are varied. In particular, the parameters may be experimentally determined values, which may be subject to noise or vary within some error interval. To test the robustness of the ODE results, it is thus necessary to study their dependence on the parameters.

In most cases, the phase space structure changes only slightly for small parameter 


\section{B. Bifurcations in dynamical systems}

changes. There are, however, certain parameter values at which a qualitative change occurs, i.e. a change in the topology of the phase space, such as the birth or death of a fixed point or a change in stability. Such points in parameter space are called bifurcation points. At a bifurcation point, the system is said to undergo a bifurcation, a qualitative change in its long-term behaviour.

Bifurcations are broadly categorized into local bifurcations, which can be identified by analysing a "small" region of phase space, and global bifurcations, which are determined by the global phase space structure. In the following I briefly discuss the local and global bifurcations that play a role in the present work, roughly following the presentation in (Strogatz, 2001).

\section{Local bifurcations}

Local bifurcations of fixed points are bifurcations that are associated with a single point in phase space. Therefore, they can be identified by observing the local neighbourhood of this point. We find local bifurcations by monitoring the stability of fixed points while continuously varying one or more parameters $\mu_{i}{ }_{1}^{1}$ Defined as a local property, the stability of a fixed point $\mathbf{x}^{*}$ can be determined by analysing the flow of trajectories in a small phase space region around $\mathbf{x}^{*}$. For this purpose, it often suffices to consider the linearization of the ODE system around the fixed point, i.e. the Jacobian $\mathbf{J}_{\mathbf{f}}$ of the vector field $\mathbf{f}$ in (B.1) evaluated at $\mathbf{x}^{*}$,

$$
\mathbf{J}_{\mathbf{f}}\left(\mathbf{x}^{*}\right)=\left.\left(\frac{\partial f_{i}}{\partial x_{j}}\right)\right|_{\mathbf{x}=\mathbf{x}^{*}} .
$$

Depending on the eigenvalue spectrum of $\mathbf{J}_{\mathbf{f}}\left(\mathbf{x}^{*}\right)$, the fixed point can be stable or unstable. If all eigenvalues of $\mathbf{J}_{\mathbf{f}}\left(\mathbf{x}^{*}\right)$ have strictly negative real parts, the fixed point is stable. Conversely, it is unstable if there are eigenvalues with strictly positive real parts. In particular, if all eigenvalues have non-zero real parts, and there are some with strictly positive and some with strictly negative real parts, the fixed point is called a saddle point. If there is an eigenvalue with vanishing real part, the stability of $\mathbf{x}^{*}$ cannot be decided from the linearized system and further non-linear terms in the expansion of the ODE must be considered.

When varying a parameter $\mu_{i}$ continuously, an eigenvalue of $\mathbf{J}_{\mathbf{f}}\left(\mathbf{x}^{*}\right)$ may cross the imaginary axis. At that point, the stability of $\mathbf{x}^{*}$ changes and a bifurcation occurs. In the present work, three common scenarios for this are of particular interest: the transcritical bifurcation, the pitchfork bifurcation, and the Hopf bifurcation. 


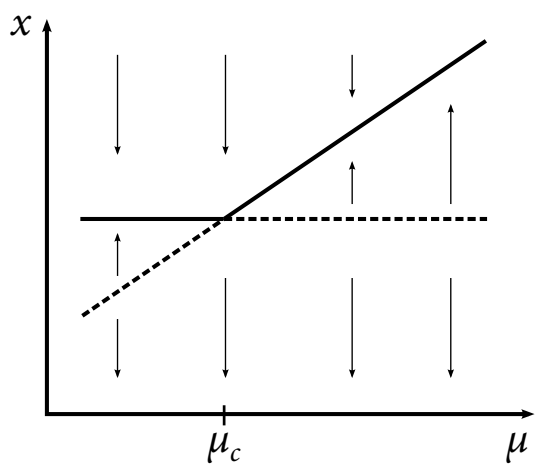

Figure B.1.: Generic form of a transcritical bifurcation. At the critical parameter value $\mu_{c}$, a stable and an unstable fixed point collide and exchange stability. Solid lines denote stable stationary states, dashed lines denote unstable ones. Arrows indicate the flow in state space.

\section{Transcritical bifurcation}

In many physical situations and in particular in the models considered in the present work, there is a stationary state, i.e. a fixed point, that exists for all values of a parameter. As the parameter is varied, the fixed point may change its stability in a transcritical bifurcation when a purely real eigenvalue of the Jacobian crosses the imaginary axis. The transcritical bifurcation can be written generically in its one-dimensional normal form

$$
\dot{x}=\mu x-x^{2}
$$

where the parameter $\mu \in \mathbb{R}$ is varied. For all values of $\mu$, there is a fixed point at $x_{0}^{*}=0$. The eigenvalue of the corresponding Jacobian at $x_{0}^{*}$ is $\mu$, so that $x_{0}^{*}$ is stable for $\mu<0$ and unstable for $\mu>0$. In addition there is an unstable fixed point at $x_{1}^{*}=\mu$ for $\mu<0$, which approaches the origin as $\mu$ is increased. At $\mu=0$, the two fixed points collide and exchange their stability: $x_{1}^{*}=\mu$ is stable for $\mu>0$ (Fig. B.1.

\section{Pitchfork bifurcation}

While the number of fixed points before and after the bifurcation is the same in the transcritical case, there are other bifurcations in which fixed points can be created or destroyed. A common such bifurcation in systems with a discrete symmetry is the pitchfork bifurcation, which can be either supercritical or subcritical.

The supercritical pitchfork bifurcation can be written generically in the normal form

$$
\dot{x}=\mu x-x^{3},
$$

\footnotetext{
${ }^{1}$ In the present work, this is done numerically in most cases using the bifurcation and continuation software auto (Doedel et al. 2009).
} 

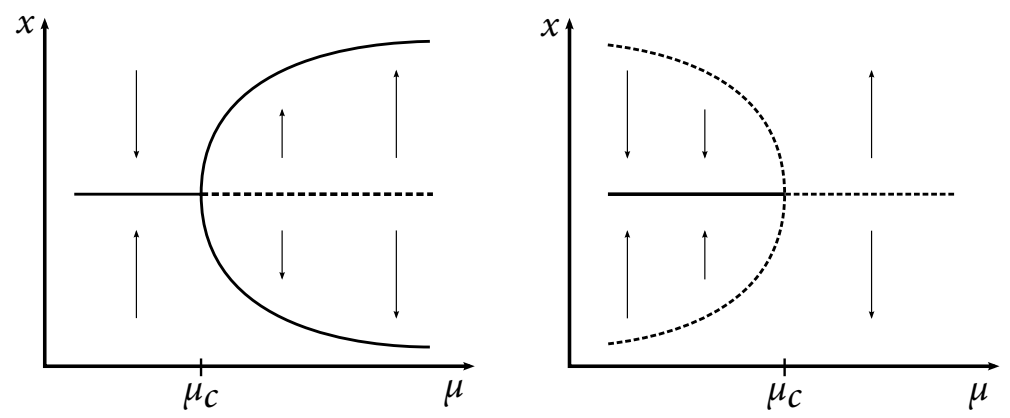

Figure B.2.: Supercritical (left) and subcritical (right) pitchfork bifurcation.

where the parameter $\mu \in \mathbb{R}$ is varied. This equation is invariant under spatial inversion, i.e. under the transformation $x \rightarrow-x$, which is a manifestation of the system's symmetry. Similar to the transcritical bifurcation, the origin $x_{0}^{*}=0$ is a fixed point for all values of $\mu$, and $\mu$ is the corresponding eigenvalue of the Jacobian. The fixed point $x_{0}^{*}$ is stable for $\mu<0$ and unstable for $\mu>0$. In contrast to the transcritical case, it is the only fixed point for $\mu<0$, while there appear two new stable fixed points $x_{1,2}^{*}= \pm \sqrt{\mu}$ for $\mu>0$. Hence, below the bifurcation all trajectories are attracted by the single fixed point $x_{0}^{*}$. Above the bifurcation, however, they approach either of the two stable fixed points $x_{1,2}^{*}$, depending on where they originate from in phase space. For $\mu>0$, the (one-dimensional) phase space is divided into two regions, where all trajectories in one region approach $x_{1}^{*}$ and all trajectories in the other region approach $x_{2}^{*}$. They are separated by the unstable fixed point $x_{0}^{*}$ (Fig. B.2. left).

The subcritical pitchfork bifurcation can be written generically in the normal form

$$
\dot{x}=\mu x+x^{3},
$$

where the cubic term now has positive sign. Here, the origin is stable for $\mu<0$ and unstable for $\mu>0$. In addition, there are two symmetric unstable fixed points $x_{1,2}^{*}= \pm \sqrt{-\mu}$ for $\mu<0$, which vanish at $\mu=0$. Consequently, there are no stable fixed points for $\mu>0$. Moreover, the cubic term leads to a blow-up of the system: for any initial condition $x(0) \neq 0$ the trajectories tend to infinity in finite time.

In most physically relevant situations, however, this blow-up is counteracted by the presence of additional, stabilizing terms. The subcritical pitchfork bifurcation is usually encountered in systems of the form

$$
\dot{x}=\mu x+x^{3}-x^{5}
$$

where the $x^{5}$-term prevents the catastrophic blow-up by creating $\mathrm{g}^{2}$ two additional stable fixed points $x_{3,4}^{*}= \pm \sqrt{(1+\sqrt{1+4 \mu}) / 2}$, which exist for all $\mu>-1 / 4$ (Fig. B.3.

\footnotetext{
${ }^{2}$ At $\mu=-1 / 4$, the four symmetric fixed points $x_{1,2}^{*}$ and $x_{3,4}^{*}$ are created in two pairs of a stable and unstable fixed point in a so-called saddle-node bifurcation.
} 


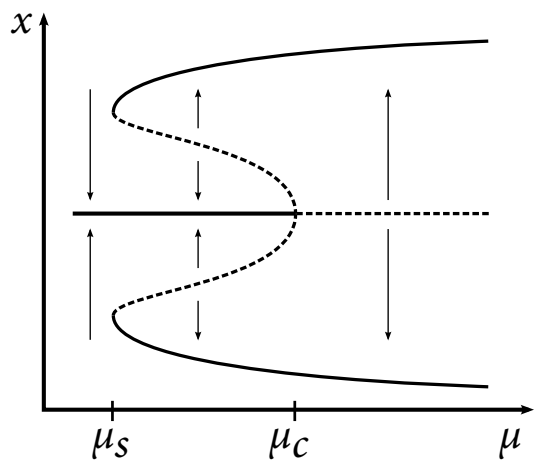

Figure B.3.: Typical scenario for the subcritical pitchfork bifurcation (at $\mu_{c}$ ). The unstable branches fold back at $\mu_{s}$ to become stable.

The subcritical pitchfork bifurcation has two remarkable properties. First, the system's long-term behaviour changes discontinuously at $\mu=0$, where the origin becomes unstable. While all the stable branches meet at $\mu=0$ in the supercritical pitchfork bifurcation, so that the two symmetric ones emerge continuously from the origin as $\mu$ is increased, this is not the case in the subcritical bifurcation. Here, a system started close to $x_{0}^{*}=0$ will eventually jump to one of the stable branches $x_{3,4}^{*}$ when $\mu$ becomes positive. Therefore, the subcritical pitchfork bifurcation is closely related to first-order phase transitions in statistical physics, while the supercritical one is related to second-order phase transitions.

The second remarkable property of the subcritical pitchfork bifurcation is the presence of bistability for $-1 / 4<\mu<0$. In this parameter region, the long-term dynamics of the system can reach one of two qualitatively different stationary states, either the origin $x_{0}^{*}$ or one of the two symmetric states $x_{3,4}^{*}$. This allows for a hysteresis loop when $\mu$ is increased beyond 0 and then decreased again down to $\mu<-1 / 4$.

\section{Hopf bifurcation}

In two- and more-dimensional dynamical systems, several other local bifurcations can occur. One of the most important examples is the Hopf bifurcation (also called AndronovHopf bifurcation), through which oscillations can appear in a dynamical system. In particular, a stable limit cycle is created in a supercritical Hopf bifurcation, while an unstable limit cycle is destroyed in a subcritical one.

The supercritical Hopf bifurcation occurs in systems of the general form

$$
\begin{aligned}
& \dot{x}=\mu x-\omega y+O\left(x^{3}\right)+O\left(y^{3}\right), \\
& \dot{y}=\omega x+\mu y+O\left(x^{3}\right)+O\left(y^{3}\right)
\end{aligned}
$$

with $\mu, \omega \in \mathbb{R}$ and $\omega \neq 0$. The system has a fixed point at the origin $x^{*}=y^{*}=0$, whose 


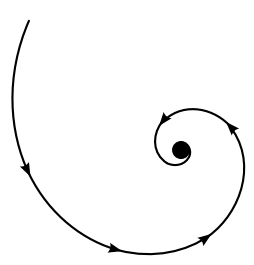

$\mu<\mu_{c}$

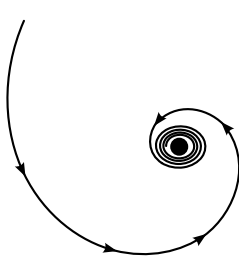

$\mu=\mu_{C}$

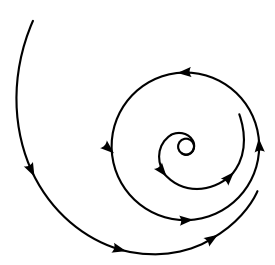

$\mu>\mu_{c}$

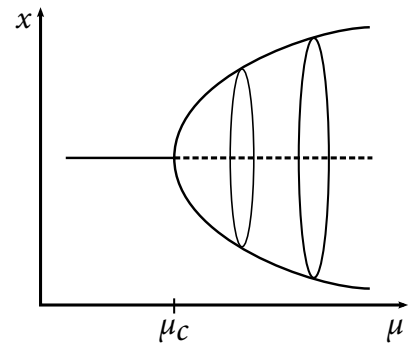

Figure B.4.: Schematic phase portrait (left) and schematic one-parameter bifurcation diagram (right) of the supercritical Hopf bifurcation (showing upper and lower turning points of the limit cycle).

stability is determined by the corresponding Jacobian

$$
\mathbf{J}(0)=\left(\begin{array}{cc}
\mu & -\omega \\
\omega & \mu
\end{array}\right)
$$

It has a pair of complex conjugate eigenvalues $\lambda=\mu \pm i \omega$, so that the origin is stable for $\mu<0$. At $\mu=0$, the two eigenvalues cross the imaginary axis simultaneously. This is the characteristic feature of a Hopf bifurcation.

As illustrated in Fig. B.4 the origin loses stability and a stable limit cycle is created in a supercritical Hopf bifurcation. For $\mu<0$, all trajectories close to the origin spiral into the fixed point $x^{*}=y^{*}=0$. For $\mu>0$, they spiral away from the now unstable origin and approach the newly created stable limit cycle that surrounds it. As $\mu$ increases further, the limit cycle grows continuously in size.

In the subcritical Hopf bifurcation, an unstable limit cycle surrounding a stable fixed point is destroyed as the latter loses stability. In analogy to the subcritical pitchfork bifurcation, this can lead to a catastrophic blow-up of the system, as the trajectories are then driven away from the now unstable fixed point.

\section{Global bifurcations}

Bifurcations that do not originate from a single point in phase space are called global bifurcations. In the present work, the only relevant global bifurcation is the homoclinic bifurcation.

\section{Homoclinic bifurcation}

Whenever a limit cycle approaches an (unstable) saddle point $\mathbf{x}^{*}$ in the phase space of a dynamical system, a homoclinic bifurcation can occur. In the schematic phase portrait in Fig. B.5. there is a stable limit cycle in the vicinity of a saddle point for a parameter value $\mu<\mu_{c}$. As $\mu$ is increased, the limit cycle grows and moves towards the saddle point 


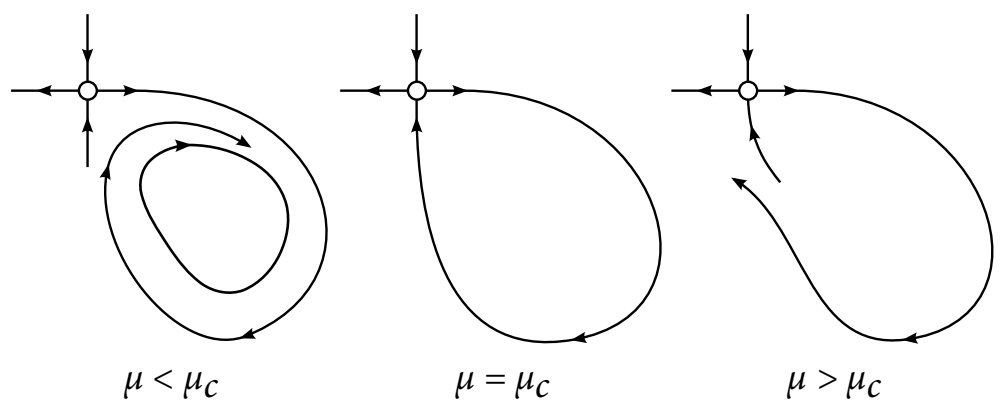

Figure B.5.: In a homoclinic bifurcation, a limit cycle collides with a saddle point, so that a homoclinic orbit is formed.

until it collides with the fixed point. At the bifurcation $\left(\mu=\mu_{c}\right)$, the cycle becomes a homoclinic orbit of $\mathbf{x}^{*}$, i.e. a closed trajectory connecting the saddle point with itself. The homoclinic orbit is a limit cycle of infinite period, so that the approach to the saddle point is infinitely slow along this trajectory. For $\mu>\mu_{c}$, the limit cycle has vanished. 



\section{The replicator equation}

\section{Reproduction dynamics}

Consider a well-mixed population of $N$ agents displaying one of $n$ phenotypes. Under reproduction dynamics, an agent of phenotype $i$ reproduces at a finite instantaneous rate $f_{i}(t)$. Hence the number $X_{i}(t)$ of agents of phenotype $i$ increases in a time interval $\Delta t$ according to

$$
X_{i}(t+\Delta t)=X_{i}(t)\left[1+f_{i}(t) \Delta t\right]
$$

and therefore

$$
\begin{aligned}
\frac{X_{i}(t+\Delta t)}{N(t+\Delta t)} & =\frac{X_{i}(t)\left[1+f_{i}(t) \Delta t\right]}{\sum_{j} X_{j}(t)\left[1+f_{j}(t) \Delta t\right]} \\
& =\frac{N(t) x_{i}(t)\left[1+f_{i}(t) \Delta t\right]}{N(t)[1+\bar{f}(t) \Delta t]},
\end{aligned}
$$

where $x_{i}(t)=X_{i}(t) / N(t)$ and $\bar{f}(t)=\sum_{j} x_{j}(t) f_{j}(t)$. Hence we have

$$
\frac{x_{i}(t+\Delta t)-x_{i}(t)}{\Delta t}=x_{i}(t) \frac{\left[f_{i}(t)-\bar{f}(t)\right]}{1+\bar{f}(t) \Delta t} .
$$

Taking the limit $\Delta t \rightarrow 0$ and dropping the time arguments, we obtain the replicator equation

$$
\dot{x}_{i}=x_{i}\left(f_{i}-\bar{f}\right) .
$$

\section{Imitation dynamics}

We obtain the same equation for imitation dynamics. In this case, an agent of strategy $i$ is imitated by other agents at a rate $f_{i}(t)$ and the population size remains constant, $N(t) \equiv N$. Thus,

$$
X_{i}(t+\Delta t)-X_{i}(t)=X_{i}(t) f_{i}(t) \Delta t \sum_{j \neq i} \frac{X_{j}(t)}{N}-X_{i}(t) \sum_{j \neq i} \frac{X_{j}(t) f_{j}(t) \Delta t}{N} .
$$

Dividing by $N \Delta t$ and taking the limit $\Delta t \rightarrow 0$, we obtain

$$
\begin{aligned}
\dot{x}_{i}(t) & =\sum_{j \neq i} x_{i}(t) x_{j}(t)\left[f_{i}(t)-f_{j}(t)\right] \\
& =x_{i}(t) \sum_{j} x_{j}(t)\left[f_{i}(t)-f_{j}(t)\right] .
\end{aligned}
$$




\section{The replicator equation}

With the normalization condition $\sum_{i} x_{i}=1$, this reduces to

$$
\dot{x}_{i}=x_{i}\left(f_{i}-\bar{f}\right),
$$

which is the same expression as (C.5). 


\section{Bibliography}

Albert, R., and A. L. Barabási (2002), Rev. Mod. Phys. 74 (1), 47

Aldana, M., V. Dossetti, C. Huepe, V. M. Kenkre, and H. Larralde (2007), Phys. Rev. Lett. $98(9), 095702$

Aldana, M., and C. Huepe (2003), J. Stat. Phys. 112 (1/2), 135

Aldana, M., H. Larralde, and B. Vázquez (2009), Int. J. Mod. Phys. B 23, 3661.

Anderson, P. W. (1972), Science 177 (4047), 393

Anstey, M. L., S. M. Rogers, S. R. Ott, M. Burrows, and S. J. Simpson (2009), Science 323 (5914), 627

Arenas, A., A. Díaz-Guilera, and C. J. Pérez-Vicente (2006), Phys. Rev. Lett. 96 (11), 114102 .

Axelrod, R. M. (1984), The evolution of cooperation (Basic Books, New York).

Axelrod, R. M., and W. D. Hamilton (1981), Science 211 (4489), 1390

Barabási, A. L. (2002), Linked: the new science of networks (Perseus Pub.).

Barabási, A. L., and R. Albert (1999), Science 286 (5439), 509

Baronchelli, A., C. Castellano, and R. Pastor-Satorras (2011), Phys. Rev. E 83 (6), 066117

Baronchelli, A., and R. Pastor-Satorras (2010), Phys. Rev. E 82 (1), 011111.

Barrat, A., M. Barthélemy, and A. Vespignani (2008), Dynamical Processes on Complex Networks (Cambridge University Press, New York, NY, USA).

Barthélemy, M., and A. Flammini (2006), J. Stat. Mech. 2006 (07), Lo7002.

Bauch, C. T. (2005), Math. Biosci. 198, 217

Bazazi, S., J. Buhl, J. J. Hale, M. L. Anstey, G. A. Sword, S. J. Simpson, and I. D. Couzin (2008), Curr. Biol. 18 (10), 735

Bednekoff, P. A. (1997), Am. Nat. 150 (3), 373

Benczik, I. J., S. Z. Benczik, B. Schmittmann, and R. K. P. Zia (2008), EPL 82 (4), 48006

van den Berg, D., and C. van Leeuwen (2004), EPL 65 (4), 459

Blasius, B., and T. Gross (2009), "Dynamic and topological interplay in adaptive networks," in Reviews of Nonlinear Dynamics and Complexity, Vol. 2, edited by H. G. Schuster, Chap. 3, 1st ed. (Wiley-VCH, Weinheim) pp. 63-106.

Blume, L. E. (1993), Games Econom. Behav. 5 (3), 387 
Bibliography

Blumstein, D. T. (1999), Science 284 (5420), 1633

Boccaletti, S., V. Latora, and Y. Moreno, Eds. (2010), Handbook on Biological Networks, World Scientific Lecture Notes in Complex Systems No. 10 (World Scientific).

Bollobás, B. (1998), Modern Graph Theory, corrected ed., Graduate texts in mathematics (Springer, Heidelberg).

Bollobás, B. (2001), Random graphs, 2nd ed., Cambridge studies in advanced mathematics No. 73 (Cambridge University Press).

Bonabeau, E., M. Dorigo, and G. Theraulaz (1999), Swarm Intelligence: From Natural to Artificial Systems, 1st ed., Santa Fe Institute Studies in the Sciences of Complexity (Oxford University Press, USA).

Boots, M., and A. Sasaki (1999), Proc. R. Soc. B 266 (1432), 1933

Borgatti, S. P., A. Mehra, D. J. Brass, and G. Labianca (2009), Science 323 (5916), 892.

Bornholdt, S., and T. Rohlf (200o), Phys. Rev. Lett. 84 (26), 6114

Bornholdt, S., and T. Röhl (2003), Phys. Rev. E 67 (6), 066118

Bourke, A. F. G., and N. R. Franks (1995), Social evolution in ants, Monographs in behavior and ecology (Princeton University Press, Princeton, NJ, USA).

Bramwell, S. T., P. C. W. Holdsworth, and J. F. Pinton (1998), Nature 396 (6711), 552

Broder, A. (2000), Computer Networks 33 (1-6), 309

Buhl, J., D. J. T. Sumpter, I. D. Couzin, J. J. Hale, E. Despland, E. R. Miller, and S. J. Simpson (2006), Science 312 (5778), 1402

Böhme, G. A., and T. Gross (2011), Phys. Rev. E 83, 035101(R)

Börner, K., S. Sanyal, and A. Vespignani (2007), Ann. Rev. Info. Sci. Tech. 41 (1), 537.

Caldarelli, G., A. Capocci, P. De Los Rios, and M. A. Muñoz (2002), Phys. Rev. Lett. 89 (25), 258702.

Camacho, J., R. Guimerà, and L. A. N. Amaral (2002), Phys. Rev. Lett. 88 (22), 228102

Castellano, C. (2005), AIP Conf. Proc. 779 (1), 114

Castellano, C., S. Fortunato, and V. Loreto (2009), Rev. Mod. Phys. 81 (2), 591.

Chaté, H., F. Ginelli, G. Grégoire, and F. Raynaud (2008), Phys. Rev. E 77 (4), 046113

Chen, Q., H. Chang, R. Govindan, and S. Jamin (2002), in INFOCOM 2002. Twenty. First Annual Joint Conference of the IEEE Computer and Communications Societies. Proceedings., Vol. 2, pp. 608-617.

Christensen, K., R. Donangelo, B. Koiller, and K. Sneppen (1998), Phys. Rev. Lett. 81 (11), 2380 .

Chu, C. Y. C., and R. D. Lee (1994), J. Pop. Econ. 7 (4), 351. 
Clauset, A., C. R. Shalizi, and M. E. J. Newman (2009), SIAM Review 51 (4), 661.

Clifford, P., and A. Sudbury (1973), Biometrika 6o (3), 581.

Conradt, L., and C. List (2009), Phil. Trans. R. Soc. B 364 (1518), 719

Cornforth, D., D. Green, D. Newth, and M. Kirley (2002), in Artificial Life VIII, edited by R. K. Standish, M. A. Bedau, and H. A. Abbass, University of New South Wales (The MIT Press) pp. 28-32.

Couzin, I. D. (2007), Nature 445 (7129), 715

Couzin, I. D., C. C. Ioannou, G. Demirel, T. Gross, C. J. Torney, A. Hartnett, L. Conradt, S. A. Levin, and N. E. Leonard (2011), Science 334 (6062), 1578

Couzin, I. D., J. Krause, N. R. Franks, and S. A. Levin (2005), Nature 433 (7025), 513

Cucker, F., and C. Huepe (2008), MathS In Action 1, 1

Cunningham, R. L. (1967), Public Choice 2 (1), 11

Dayan, P., and L. F. Abbott (2005), Theoretical neuroscience (The MIT Press).

Deffuant, G., D. Neau, F. Amblard, and G. Weisbuch (2000), Adv. Complex Syst. 3 (1-4): 87.

Demirel, G., R. Prizak, P. N. Reddy, and T. Gross (2011a), Eur. Phys. J. B 84 (4), 541

Demirel, G., F. Vazquez, G. A. Böhme, and T. Gross (2011b), "Moment closure approximations of discrete-state adaptive networks," in prep.

Derrida, B., E. Gardner, and A. Zippelius (1987), EPL 4 (2), 167

Diestel, R. (2005), Graph Theory, 3rd ed., Graduate Texts in Mathematics No. 173 (Springer, Heidelberg).

Do, A.-L., and T. Gross (2009), in Gross and Sayama (2009), Chap. 9, pp. 191-208.

Do, A.-L., L. Rudolf, and T. Gross (2010), New J. Phys. 12, 063023

Doebeli, M., and C. Hauert (2005), Ecol. Lett. 8, 748

Doedel, E., A. Champneys, F. Dercole, T. Fairgrieve, Y. Kuznetsov, B. Oldeman, R. Paffenroth, B. Sandstede, X. Wang, and C. Zhang (2009), Auto: Software for continuation and bifurcation problems in ordinary differential equations, Tech. Rep. (Concordia University, Montreal).

Dornic, I., H. Chaté, J. Chave, and H. Hinrichsen (2001), Phys. Rev. Lett. 87 (4), 045701.

Dorogovtsev, S. N., A. V. Goltsev, and J. F. F. Mendes (2002), Phys. Rev. E 66 (1), 016104

Dorogovtsev, S. N., A. V. Goltsev, and J. F. F. Mendes (2008), Rev. Mod. Phys. 8o (4), 1275.

Dorogovtsev, S. N., J. F. F. Mendes, and A. N. Samukhin (2001), Phys. Rev. E 64 (2), 025101

Dossetti, V., F. J. Sevilla, and V. M. Kenkre (2009), Phys. Rev. E 79 (5), 051115. 


\section{Bibliography}

Ebel, H., and S. Bornholdt (2002), "Evolutionary games and the emergence of complex networks," arXiv:cond-mat/o211666

Eftimie, R., G. de Vries, and M. A. Lewis (2007), Proc. Natl. Acad. Sci. U. S. A. 104 (17), 6974

Eguíluz, V. M., M. G. Zimmermann, C. J. Cela-Conde, and M. San Miguel (2005), Am. J. Sociol. 110 (4), 977.

Erdős, P., and A. Rényi (1959), Publ. Math. (Debrecen) 6, 290.

Erdős, P., and A. Rényi (1960), Publ. Math. Inst. Hung. Acad. Sci. 5, 17.

Escudero, C., C. A. Yates, J. Buhl, I. D. Couzin, R. Erban, I. G. Kevrekidis, and P. K. Maini (2010), Phys. Rev. E 82 (1), 011926

Euler, L. (1736), Commentarii Academiae Scientiarum Imperialis Petropolitanae 8, 128.

Feld, S. L. (1991), Am. J. Sociol. 96 (6), 1464

Flaounas, I., M. Turchi, O. Ali, N. Fyson, T. D. Bie, N. Mosdell, J. Lewis, and N. Cristianini (2010), PLoS ONE 5 (12), e14243

Frank, S. A. (2009), in Levin (2009), pp. 3-40.

Franks, N. R. (1989), Am. Sci. 77 (2), 139

Freeman, L. C. (2004), The Development of Social Network Analysis: A Study in the Sociology of Science (Empirical Press, Vancouver, Canada).

Fromm, J. (2004), The Emergence of Complexity (kassel university press, Kassel).

Funk, S., E. Gilad, C. Watkins, and V. A. A. Jansen (2009), Proc. Natl. Acad. Sci. U. S. A. $106(16), 6872$

Funk, S., M. Salathé, and V. A. A. Jansen (2010), J. R. Soc. Interface 7, 1247

Gil, S., and D. Zanette (2006), Phys. Lett. 356 (2), 89

Gillespie, D. T. (1976), J. Comput. Phys. 22, 403.

Gong, P., and C. van Leeuwen (2004), EPL 67 (2), 328

Gore, J., H. Youk, and A. van Oudenaarden (2009), Nature 459, 253

Gross, T., and B. Blasius (2008), J. R. Soc. Interface 5 (20), 259

Gross, T., C. J. D. D'Lima, and B. Blasius (2006), Phys. Rev. Lett. 96 (20), 208701

Gross, T., and I. G. Kevrekidis (2008), EPL 82 (3), 38004

Gross, T., L. Rudolf, S. A. Levin, and U. Dieckmann (2009), Science 325 (5941), 747

Gross, T., and H. Sayama, Eds. (2009), Adaptive networks: Theory, Models and Applications Understanding Complex Systems (Springer, New York).

Gräser, O., C. Xu, and P. M. Hui (2009), EPL 87 (3), 38003. 
Grégoire, G., and H. Chaté (2004), Phys. Rev. Lett. 92 (2), 025702.

Guckenheimer, J., and P. Holmes (2002), Nonlinear oscillations, dynamical systems, and bifurcations of vector fields, 7 th ed., Applied mathematical sciences No. 42 (Springer, New York).

Guerra, B., and J. Gómez-Gardeñes (2010), Phys. Rev. E 82 (3), 035101

Gómez-Gardeñes, J., and Y. Moreno (2006), Phys. Rev. E 73 (5), 056124

Hamilton, W. D. (1964), J. Theor. Biol. 7 (1), 1.

Hammerstein, P., Ed. (2003), Genetic and Cultural Evolution of Cooperation, Dahlem Workshop Reports (The MIT Press, Cambridge, MA).

Hanaki, N., A. Peterhansl, P. S. Dodds, and D. J. Watts (2007), Mgmt. Sci. 53 (7), 1036

Hauert, C. (2002), Int. J. Bifurcat. Chaos 12 (7), 1531.

Hauert, C., S. D. Monte, J. Hofbauer, and K. Sigmund (2002), Science 296 (5570), 1129

Hensinger, E., I. Flaounas, and N. Cristianini (2010), in Artificial Intelligence Applications and Innovations IFIP Advances in Information and Communication Technology, Vol. 339, edited by H. Papadopoulos, A. Andreou, and M. Bramer, Chap. 25 (Springer Boston, Berlin, Heidelberg) pp. 179-186.

Hethcote, H. W. (2000), SIAM Review 42 (4), 599.

Hinrichsen, H. (2006), Physica A 369 (1), 1.

Hofbauer, J., and K. Sigmund (1998), Evolutionary Games and Population Dynamics (Cambridge University Press, Cambridge).

Holley, R. A., and T. M. Liggett (1975), Ann. Probab. 3 (4), 643.

Holme, P., and B. J. Kim (2002), Phys. Rev. E 65 (2), 026107

Holme, P., and M. E. J. Newman (2006), Phys. Rev. E 74 (5), 056108

House, T., G. Davies, L. Danon, and M. Keeling (2009), Bull. Math. Biol. 71 (7), 1693

House, T., and M. J. Keeling (2011), J. R. Soc. Interface 8 (54), 67.

Hu, Y., M. Li, P. Zhang, Y. Fan, and Z. Di (2008), Phys. Rev. E 78 (1), 016115

Huberman, B. A., and N. S. Glance (1993), Proc. Natl. Acad. Sci. U. S. A. 90 (16), 7716

Huepe, C., and M. Aldana (2004), Phys. Rev. Lett. 92 (16), 168701

Huepe, C., and M. Aldana (2008), Physica A 387 (12), 2809

Huepe, C., G. Zschaler, A.-L. Do, and T. Gross (2011), New J. Phys. 13, 073022.

Hébert-Dufresne, L., P.-A. Noël, V. Marceau, A. Allard, and L. J. Dubé (2010), Phys. Rev. E 82 (3), 036115

Ilachinski, A. (2001), Cellular Automata: A Discrete Universe (World Scientific, Singapore). 


\section{Bibliography}

Imhof, L. A., D. Fudenberg, and M. A. Nowak (2005), Proc. Natl. Acad. Sci. U. S. A. $102(31), 10797$

Ito, J., and K. Kaneko (2001), Phys. Rev. Lett. 88 (2), 028701.

Jiang, L., D. Hua, J. Zhu, B. Wang, and T. Zhou (2008), Eur. Phys. J. B 65, 251

van Kampen, N. G. (1997), Stochastic Processes in Physics and Chemistry, 2nd ed. (Elsevier Science, Amsterdam).

Kappeler, P., and C. van Schaik, Eds. (2006), Cooperation in Primates and Humans (Springer, Berlin Heidelberg).

Karrer, B., and M. E. J. Newman (2010), Phys. Rev. E 82 (1), 016101

Kauffman, S. (1969), J. Theor. Biol. 22 (3), 437

Keeling, M. J., and K. T. D. Eames (2005), J. R. Soc. Interface 2, 295.

Keeling, M. J., D. A. Rand, and A. J. Morris (1997), Proc. R. Soc. B 264, 1149

Keller, E. F. (2005), Bioessays 27 (10), 1060

Kimura, D., and Y. Hayakawa (2008), Phys. Rev. E 78 (1), 016103.

Klemm, K., and V. M. Eguíluz (2002a), Phys. Rev. E 65 (5), 057102.

Klemm, K., and V. M. Eguíluz (2002b), Phys. Rev. E 65 (3), 036123

Konno, T. (2011), J. Theor. Biol. 269 (1), 224

Kun, Á., and I. Scheuring (2009), Biosystems 96 (1), 65

Kuperman, M., and G. Abramson (2001), Phys. Rev. Lett. 86 (13), 2909

Kuramoto, Y. (2003), Chemical oscillations, waves, and turbulence, Chemistry Series (Dover Publications) originally published: Springer Berlin, New York, Heidelberg, 1984 .

Kuznetsov, Y. A. (1998), Elements of applied bifurcation theory, 2nd ed., Applied Mathematical Sciences No. 112 (Springer, Berlin).

Kümmerli, R., C. Colliard, N. Fiechter, B. Petitpierre, F. Russier, and L. Keller (2007), Proc. R. Soc. B 274 (1628), 2965

Lambiotte, R., and J. C. González-Avella (2011), Physica A 390 (2), 392

Levin, S. A., Ed. (2009), Games, Groups, and the Global Good, Springer Series in Game Theory (Springer, Berlin).

Liggett, T. M. (1999), Stochastic interacting systems: contact, voter, and exclusion processes, Vol. 324 (Springer, Berlin Heidelberg New York).

Liljeros, F., C. R. Edling, L. A. Amaral, H. E. Stanley, and Y. Aberg (2001), Nature $411(6840), 907$

Liu, M., and K. E. Bassler (2006), Phys. Rev. E 74 (4), 041910. 
Lucas, J. R. (1959), Philosophy 34 (128), 1.

Lukeman, R., Y.-X. Li, and L. Edelstein-Keshet (2010), Proc. Natl. Acad. Sci. U. S. A. $107(28), 12576$.

Mach, R., and F. Schweitzer (2007), Bull. Math. Biol. 69 (2), 539

Maddow, R. (2010), “The Rachel Maddow show," MSNBC TV show, critical comment on U.S. news reports that supported their claim that the president's visit to India cost $\$ 200$ million per day by citing other reports in the news and on the Internet, http://www.msnbc.msn.com/id/3096434/\#40018314

Marceau, V., P.-A. Noël, L. Hébert-Dufresne, A. Allard, and L. J. Dubé (2010), Phys. Rev. E 82 (3), 036116

Masuda, N., and H. Ohtsuki (2009), New J. Phys. 11 (3), 033012

Maynard Smith, J. (1982), Evolution and the Theory of Games (Cambridge University Press).

Maynard Smith, J., and G. R. Price (1973), Nature 246 (5427), 15

McPherson, M., L. Smith-Lovin, and J. M. Cook (2001), Annu. Rev. Sociol. 27 (1), 415

Meisel, C., and T. Gross (2009), Phys. Rev. E 80 (6), 061917

Milgram, S. (1967), Psychology Today 1 (1), 61.

Mitchell, M. (2009), Complexity: A Guided Tour (Oxford University Press, USA).

Mobilia, M., A. Petersen, and S. Redner (2007), J. Stat. Mech. 2007 (08), Po8029

Moreno, Y., and A. F. Pacheco (2004), EPL 68 (4), 603

Morris, A. J. (1997), Representing Spatial Interactions in Simple Ecological Models, Ph.D. thesis (University of Warwick).

Nagy, M., I. Daruka, and T. Vicsek (2007), Physica A 373, 445

Nardini, C., B. Kozma, and A. Barrat (2008), Phys. Rev. Lett. 100, 158701

von Neumann, J., and O. Morgenstern (1944), Theory of Games and Economic Behavior (Princeton University Press, Princeton, NJ).

Newman, M., A. L. Barabási, and D. J. Watts (2006), The Structure and Dynamics of Networks (Princeton University Press).

Newman, M. E. J. (2003), SIAM Review 45 (2), 167

Newman, M. E. J. (2010), Networks: An Introduction (Oxford University Press).

Newman, M. E. J., S. H. Strogatz, and D. J. Watts (2001), Phys. Rev. E 64 (2), 026118

Newth, D., and D. Cornforth (2009), Biosystems 95 (2), 120

Noh, J. D., and H. Rieger (2004), Phys. Rev. Lett. 92 (11), 118701

Nowak, M., and K. Sigmund (1993), Nature 364 (6432), 56. 


\section{Bibliography}

Nowak, M. A. (2006), Science 314 (5805), 1560

Nowak, M. A., and R. M. May (1992), Nature 359, 826

Nowak, M. A., A. Sasaki, C. Taylor, and D. Fudenberg (2004), Nature 428 (6983), 646

Nowak, M. A., and K. Sigmund (1989), J. Theor. Biol. 137 (1), 21

Nowak, M. A., and K. Sigmund (1998), Nature 393 (6685), 573

Nowak, M. A., and K. Sigmund (2005), Nature 437, 1291

Ohtsuki, H., C. Hauert, E. Lieberman, and M. A. Nowak (2006), Nature 441 (7092), 502

Ohtsuki, H., and M. A. Nowak (2006), J. Theor. Biol. 243 (1), 86

Ohtsuki, H., M. A. Nowak, and J. M. Pacheco (2007), Phys. Rev. Lett. 98 (10), 108106.

Osborne, M. J., and A. Rubinstein (1994), A course in game theory (The MIT Press, Cambridge, USA) electronic edition.

Pacheco, J. M., A. Traulsen, and M. A. Nowak (2006a), J. Theor. Biol. 243 (3), 437

Pacheco, J. M., A. Traulsen, and M. A. Nowak (2006b), Phys. Rev. Lett. 97 (25), 258103

Park, S. M., and B. J. Kim (2006), Phys. Rev. E 74 (2), 026114

Pastor-Satorras, R., and A. Vespignani (2001a), Phys. Rev. E 63, 066117

Pastor-Satorras, R., and A. Vespignani (2001b), Phys. Rev. Lett. 86 (14), 3200

Pennisi, E. (2009), Science 325 (5945), 1196

Perc, M., and A. Szolnoki (2010), Biosystems 99 (2), 109

Peyrard, N., U. Dieckmann, and A. Franc (2008), Theoretical Population Biology 73 (3), 383.

Pimentel, J. A., M. Aldana, C. Huepe, and H. Larralde (2008), Phys. Rev. E 77 (6), 061138

Pimm, S. L. (2002), Food webs (University of Chicago Press).

Poncela, J., J. Gómez-Gardeñes, L. M. Floría, Y. Moreno, and A. Sánchez (2009a), EPL $88(3), 38003$.

Poncela, J., J. Gómez-Gardeñes, L. M. Floría, A. Sánchez, and Y. Moreno (2008), PLoS ONE 3 (6), e2449.

Poncela, J., J. Gómez-Gardeñes, A. Traulsen, and Y. Moreno (2009b), New J. Phys. 11 (8), 083031

Portelli, B., P. Holdsworth, and J. F. Pinton (2003), Phys. Rev. Lett. 90 (10), 104501

Press, W. H., S. A. Teukolsky, W. T. Vetterling, and B. P. Flannery (2007), Numerical Recipes: The Art of Scientific Computing, 3rd ed. (Cambridge University Press).

Pugliese, E., and C. Castellano (2009), EPL 88 (5), 58004

Rand, D. A. (1999), in CWI Quarterly, Vol. 12 (3\&4), edited by J. M. McGlade, Centrum 
Wiskunde \& Informatica (Wiley-Blackwell) pp. 329-368.

Ratnieks, F. L. W., T. Monnin, and K. R. Foster (2001), Ann. Zool. Fennici 38, 201-214

Reichardt, J., and S. Bornholdt (2004), Phys. Rev. Lett. 93 (21), 218701.

Reichenbach, T., M. Mobilia, and E. Frey (2006), Phys. Rev. E 74 (5), 051907

Ren, J., X. Wu, W.-X. Wang, G. Chen, and B.-H. Wang (2006), "Interplay between evolutionary game and network structure: the coevolution of social net, cooperation and wealth," arXiv:physics/0605250

Renshaw, E. (1991), Modelling biological populations in space and time, Cambridge Studies in Mathematical Biology (Cambridge University Press).

Reynolds, C. W. (1987), SIGGRAPH Comput. Graph. 21 (4), 25

Roca, C. P., J. A. Cuesta, and A. Sánchez (2009), Phys. Life Rev. 6 (4), 208

Romanczuk, P., I. D. Couzin, and L. Schimansky-Geier (2009), Phys. Rev. Lett. 102 (1), 010602

Ross, D. (2010), “Game theory," The Stanford Encyclopedia of Philosophy (Fall 2010).

Rozhnova, G., and A. Nunes (2009), Phys. Rev. E 79, 041922

Santos, F. C., and J. M. Pacheco (2005), Phys. Rev. Lett. 95, 098104

Santos, F. C., J. M. Pacheco, and T. Lenaerts (2006a), PLoS Comput. Biol. 2 (10), e140.

Santos, F. C., J. M. Pacheco, and T. Lenaerts (2006b), Proc. Natl. Acad. Sci. U. S. A. 103 (9). 3490

Sayama, H., and C. Laramee (2009), in Gross and Sayama (2009), Chap. 15, pp. 311-332.

Schelling, T. C. (1969), Am. Econ. Rev. 59 (2), 488

Schelling, T. C. (1978), Micromotives and Macrobehavior (W. W. Norton \& Company) reissue 2006.

Sedgewick, R. (2002), Algorithms in C++, 3rd ed., Vol. 5 (Addison Wesley).

van Segbroeck, S., F. C. Santos, T. Lenaerts, and J. M. Pacheco (2009), Phys. Rev. Lett. 102, 058105 .

van Segbroeck, S., F. C. Santos, T. Lenaerts, and J. M. Pacheco (2011), New J. Phys. 13 (1), 013007 .

Ángeles Serrano, M., K. Klemm, F. Vazquez, V. M. Eguíluz, and M. S. Miguel (2009), J. Stat. Mech. 2009 (10), P10024.

Siek, J., L.-Q. Lee, and A. Lumsdaine (2002), The Boost Graph Library: user guide and reference manual $\mathrm{C}++$ in-depth series (Addison-Wesley).

Sigmund, K. (2007), Trends Ecol. Evol. 22 (11), 593

Sigmund, K., C. Hauert, and M. A. Nowak (2001), Proc. Natl. Acad. Sci. U. S. A. 98 (19), 
10757 .

Simon, H. A. (1955), Biometrika 42 (3-4), 425

Simpson, S. J., E. Despland, B. F. Hägele, and T. Dodgson (2001), Proc. Natl. Acad. Sci. U. S. A. $98(7), 3895$

Simpson, S. J., A. R. McCaffery, and B. F. Hägele (1999), Biol. Rev. 74 (4), 461.

Simpson, S. J., G. A. Sword, P. D. Lorch, and I. D. Couzin (2006), Proc. Natl. Acad. Sci. U. S. A. 103 (11), 4152.

Singer, A. (2004), J. Chem. Phys. 121 (8), 3657

de Solla Price, D. J. (1965), Science 149 (3683), 510

Sood, V., T. Antal, and S. Redner (2008), Phys. Rev. E 77 (4), 041121

Sood, V., and S. Redner (2005), Phys. Rev. Lett. 94 (17), 178701

Standish, R. K. (2008), in Intelligent Complex Adaptive Systems, edited by A. Yang, A. Yang, and Y. Shan, Chap. 4 (IGI Publishing, Hershey, PA, USA) p. 105, arXiv:0805.0685V1

Strefler, J., U. Erdmann, and L. Schimansky-Geier (2008), Phys. Rev. E 78 (3), 031927.

Strogatz, S. H. (2001), Nonlinear Dynamics And Chaos: With Applications To Physics, Biology, Chemistry, And Engineering, 1st ed., Studies in Nonlinearity (Westview Press).

Stumpf, M. P. H., C. Wiuf, and R. M. May (2005), Proc. Natl. Acad. Sci. U. S. A. 102 (12), 4221.

Suchecki, K., V. M. Eguíluz, and M. S. Miguel (2005a), EPL 69, 228

Suchecki, K., V. M. Eguíluz, and M. S. Miguel (2005b), Phys. Rev. E 72 (3), 036132

Sugden, R. (1986), The economics of rights, co-operation and welfare (B. Blackwell, Oxford).

Sumpter, D. J. T., J. Buhl, D. Biro, and I. D. Couzin (2008), Theory in Biosciences 127 (2), 177

Suzuki, R., M. Kato, and T. Arita (2008), Phys. Rev. E 77 (2), 021911

Szabó, G., and G. Fáth (2007), Phys. Rep. 446 (4-6), 97.

Szabó, G., and C. Tőke (1998), Phys. Rev. E 58 (1), 69

Szolnoki, A., and M. Perc (2009a), New J. Phys. 11 (9), 093033

Szolnoki, A., and M. Perc (2009b), EPL 86, 30007

Szolnoki, A., M. Perc, and Z. Danku (2008), EPL 84 (5), 50007.

Sánchez, A. D., J. M. López, and M. A. Rodríguez (2002), Phys. Rev. Lett. 88 (4), 048701

Tadić, B., S. Thurner, and G. J. Rodgers (2004), Phys. Rev. E 69 (3), 036102.

Tomita, K., H. Kurokawa, and S. Murata (2002), Physica D 171 (4), 197

Tomita, K., H. Kurokawa, and S. Murata (2009), in Gross and Sayama (2009), Chap. 14, 
pp. 291-309.

Tomita, K., S. Murata, and H. Kurokawa (2007), in Advances in Artificial Life. Lecture Notes in Computer Science, Vol. 4648, edited by F. Almeida e Costa, L. M. Rocha, E. Costa, I. Harvey, and A. Coutinho, Chap. 87 (Springer, Berlin, Heidelberg) pp. 865-875.

Toner, J., and Y. Tu (1995), Phys. Rev. Lett. 75 (23), 4326.

Toner, J., and Y. Tu (1998), Phys. Rev. E 58 (4), 4828

Topaz, C. M., and A. L. Bertozzi (2004), SIAM J. Appl. Math. 65 (1), 152

Traulsen, A., M. A. Nowak, and J. M. Pacheco (2006), Phys. Rev. E 74 (1), 011909.

Traulsen, A., D. Semmann, R. D. Sommerfeld, H.-J. Krambeck, and M. Milinski (2010), Proc. Natl. Acad. Sci. U. S. A. 107 (7), 2962

Turchin, P. (2003), Historical Dynamics: Why States Rise and Fall, Princeton Studies in Complexity (Princeton University Press).

Vazquez, F., and V. M. Eguíluz (2008), New J. Phys. 10 (6), 063011

Vazquez, F., V. M. Eguíluz, and M. S. Miguel (2008), Phys. Rev. Lett. 100 (10), 108702

Vicsek, T., A. Czirók, E. Ben-Jacob, I. Cohen, and O. Shochet (1995), Phys. Rev. Lett. $75(6), 1226$

Wasserman, S., and K. Faust (1994), Social Network Analysis: Methods and Applications, 1st ed., Structural analysis in the social sciences No. 8 (Cambridge University Press).

Watts, D. J. (2003), Six Degrees: The Science of a Connected Age, 1st ed. (W. W. Norton \& Company).

Watts, D. J., and S. H. Strogatz (1998), Nature 393 (6684), 440

Webb, A. P., Ed. (2006), Profiting from Proliferation (McKinsey \& Co., New York).

Weibull, J. W. (1997), Evolutionary Game Theory (The MIT Press).

West, S. A., S. P. Diggle, A. Buckling, A. Gardner, and A. S. Griffin (2007), Annu. Rev. Ecol. Evol. Syst. 38 (1), 53

West, S. A., I. Pen, and A. S. Griffin (2002), Science 296 (5565), 72.

Yates, C. A., R. Erban, C. Escudero, I. D. Couzin, J. Buhl, I. G. Kevrekidis, P. K. Maini, and D. J. T. Sumpter (2009), Proc. Natl. Acad. Sci. U. S. A. 106 (14), 5464

Yule, G. U. (1944), The Statistical Study of Literary Vocabulary (Cambridge University Press).

Zimmermann, M. G., and V. M. Eguíluz (2005), Phys. Rev. E 72 (5), 056118

Zimmermann, M. G., V. M. Eguíluz, and M. San Miguel (2001), in Economics with Heterogeneous Interacting Agents, Lecture Notes in Economics and Mathematical Systems, edited by A. Kirman and J. Zimmermann (Springer) p. 73. 


\section{Bibliography}

Zimmermann, M. G., V. M. Eguíluz, and M. San Miguel (2004), Phys. Rev. E 69, 065102

Zimmermann, M. G., V. M. Eguíluz, M. San Miguel, and A. Spadaro (2000), Adv. Complex Syst. 3, 283

Zschaler, G., G. A. Böhme, M. Seißinger, C. Huepe, and T. Gross (2012), Phys. Rev. E 69, 046107.

Zschaler, G., A. Traulsen, and T. Gross (2010), New J. Phys. 12 (9), 093015 


\section{Acknowledgements}

I would like to thank my supervisor Dr. Thilo Gross for arousing my interest in this work and patiently accompanying my often rather slow progress. I greatly benefited from his constant supply of stimulating ideas, his experience and comprehensive knowledge of the field, and his ability to generate motivation out of thin air.

I also thank my colleagues in the research group Dynamics of Biological Networks for the many fruitful discussions, collaborations, and for making the past years such an enjoyable experience.

Furthermore, I would like to thank Prof. Dr. Frank Jülicher for giving me the opportunity to work in the Biological Physics department at the MPIPKS. I greatly appreciate the interdisciplinary and open character of this institute, which stimulates to think outside the box and has allowed me to meet many interesting people.

Last but not least, I thank my partner Frank Winkler, without whom I would not be complete, and my parents for their loving and generous support, unobtrusive interest, and for always believing in me. 



\section{Versicherung}

Hiermit versichere ich, dass ich die vorliegende Arbeit ohne unzulässige Hilfe Dritter und ohne Benutzung anderer als der angegebenen Hilfsmittel angefertigt habe; die aus fremden Quellen direkt oder indirekt übernommenen Gedanken sind als solche kenntlich gemacht. Die Arbeit wurde bisher weder im Inland noch im Ausland in gleicher oder ähnlicher Form einer anderen Prüfungsbehörde vorgelegt.

Die Dissertation wurde in der Zeit von August 2008 bis Dezember 2011 unter der Betreuung von Dr. Thilo Gross und Prof. Dr. Frank Jülicher in der Arbeitsgruppe „Dynamik biologischer Netzwerke“ am Max-Planck-Institut für Physik komplexer Systeme in Dresden angefertigt.

Dresden, im Dezember 2011 